$\frac{10 / 3}{31-968 \delta 0}$

SANDIA REPORT

SAND96-8218 • UC-405

- Unlimited Release

Printed February 1996

\title{
Aurora: A Fortran Program for Modeling Well Stirred Plasma and Thermal Reactors with Gas and Surface Reactions
}

Ellen Meeks, Harry K. Moffat, Joseph F. Grcar, and Robert J. Kee

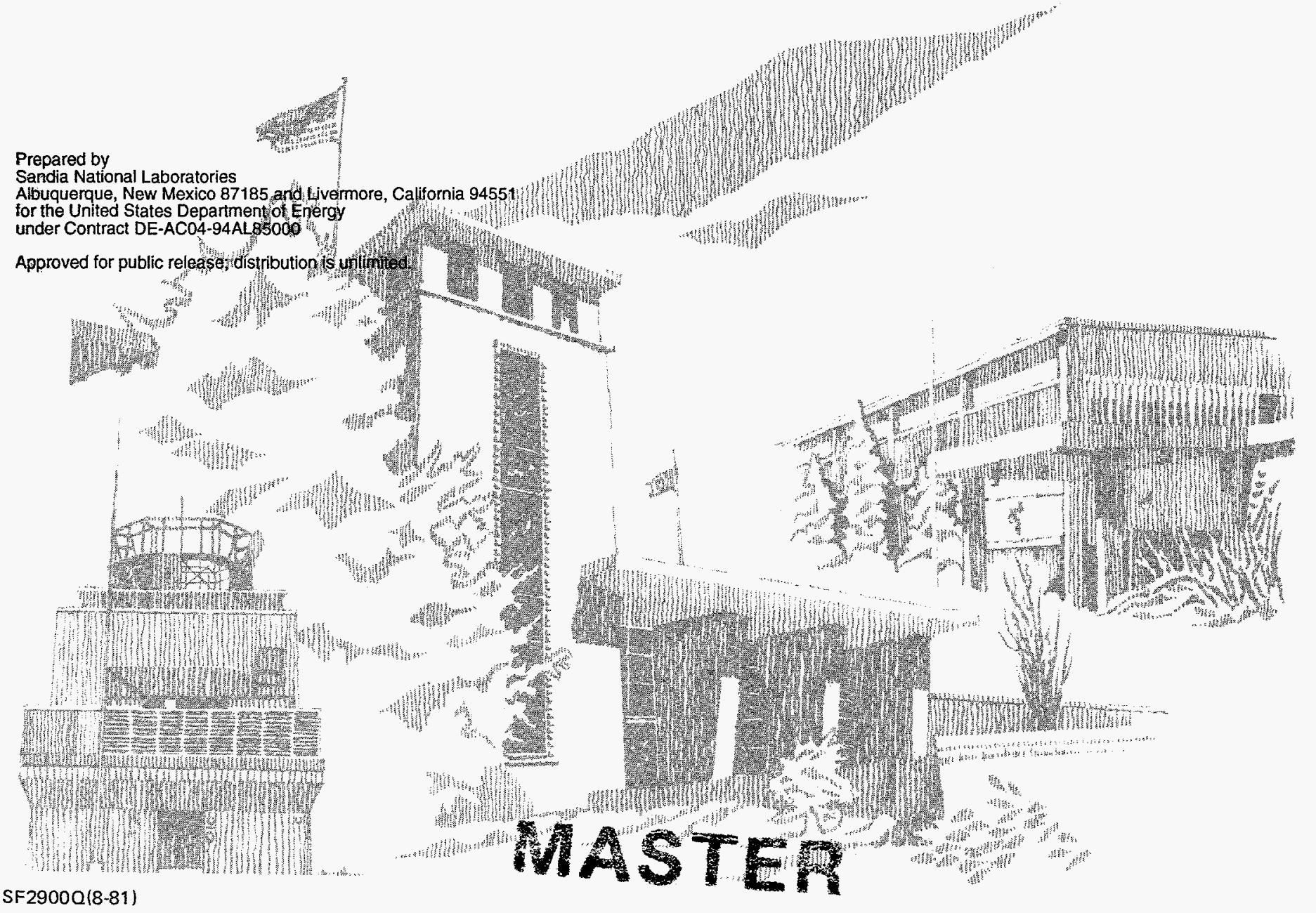




\begin{abstract}
Issued by Sandia National Laboratories, operated for the United States Department of Energy by Sandia Corporation.

NOTICE: This report was prepared as an account of work sponsored by an agency of the United States Government. Neither the United States Government nor any agency thereof, nor any of their employees, nor any of the contractors, subcontractors, or their employees, makes any war. ranty, express or implied, or assumes any legal liability or responsibility for the accuracy, completeness, or usefulness of any information, apparatus, product, or process disclosed, or represents that its use would not infringe privately owned rights. Reference herein to any specific commercial product, process, or service by trade name, trademark, manufacturer, or otherwise, does not necessarily constitute or imply its endorsement, recommendation, or favoring by the United States Government, any agency thereof or any of their contractors or subconractors. The views and opinions expressed herein do not necessarily state or reflect those of the United States Government, any agency thereof or any of their contractors or subcontractors.
\end{abstract}

This report has been reproduced from the best available copy.

Available to DOE and DOE contractors from:

Office of Scientific and Technical Information

P. O. Box 62

Oak Ridge, TN 37831

Prices available from (615) 576-8401, FTS 626-8401

Available to the public from:

National Technical Information Service

U.S. Department of Commerce

5285 Port Royal Rd.

Springfield, VA 22161 
DISClamiar

Portions of this document may be illegible in electronic image products. Images are produced from the best available original document. 
UC-405

SAND96-8218

Unlimited Release

Printed February 1996

\title{
AURORA: A FORTRAN PROGRAM FOR MODELING WELL STIRRED PLASMA AND THERMAL REACTORS WITH GAS AND SURFACE REACTIONS
}

\author{
Ellen Meeks, Joseph F. Grcar, and Robert J. Kee \\ Thermal and Plasma Processes Department \\ Sandia National Laboratories \\ Livermore, CA 94551-0939 \\ Harry K. Moffat \\ Surface Processing Sciences Department \\ Sandia National Laboratories \\ Albuquerque, NM 87185
}

\begin{abstract}
The AURORA Software is a FORTRAN computer program that predicts the steady-state or timeaveraged properties of a well mixed or perfectly stirred reactor for plasma or thermal chemistry systems. The software was based on the previously released software, SURFACE PSR, ${ }^{1}$ which was written for application to thermal CVD reactor systems. AURORA allows modeling of non-thermal, plasma reactors with the determination of ion and electron concentrations and the electron temperature, in addition to the neutral radical species concentrations. Well stirred reactors are characterized by a reactor volume, residence time or mass flow rate, heat loss or gas temperature, surface area, surface temperature, the incoming temperature and mixture composition, as well as the power deposited into the plasma for non-thermal systems. The model described here accounts for finite-rate elementary chemical reactions both in the gas phase and on the surface. The governing equations are a system of nonlinear algebraic relations. The program solves these equations using a hybrid Newton/timeintegration method embodied by the software package TWOPNT. The program runs in conjunction with the new CHEMKIN-III ${ }^{2}$ and SURFACE CHEMKIN-III ${ }^{3}$ packages, which handle the chemical reaction mechanisms for thermal and non-thermal systems. CHEMKIN-III allows for specification of electronimpact reactions, excitation losses, and elastic-collision losses for electrons.
\end{abstract}




\section{ACKNOWLEDGMENTS}

The development of the AURORA code has been supported in part by a cooperative research agreement between the U. S. Department of Energy and SEMATECH, contract number DE-F10489AL58872. 


\section{CONTENTS}

LIST OF FIGURES

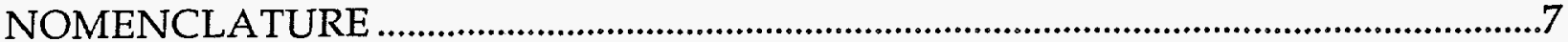

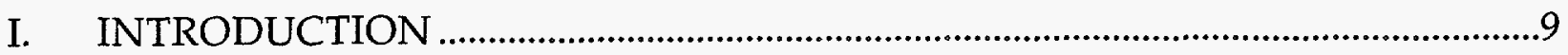

II. WELL MIXED REACTOR EQUATIONS..................................................................11

Mass Conservation and Gas-Phase Species Equations............................................................... 12

Surface Species Equations............................................................................................ 13

Bulk Species Equations During Deposition............................................................................. 15

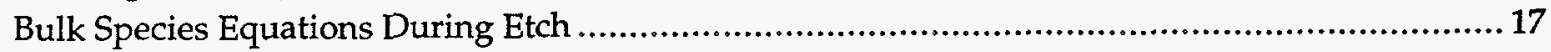

Non-Constant Surface-Phase Site Densities ............................................................... 17

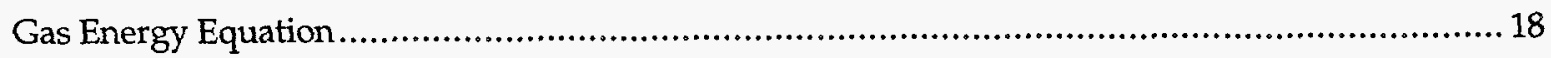

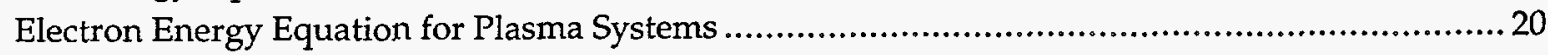

Gas Energy Equation Adjusted for Plasma Systems.......................................................... 22

Application of the Bohm Condition for Ion Fluxes to Surfaces ......................................... 22

Summary of Solution Variables .............................................................................. 23

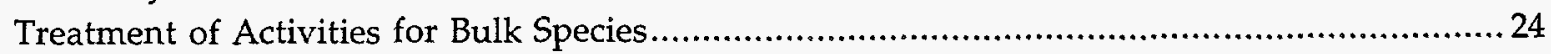

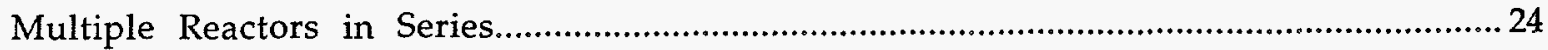

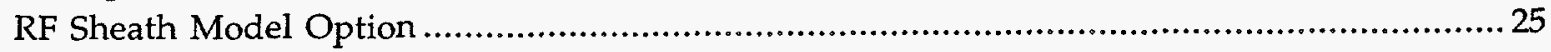

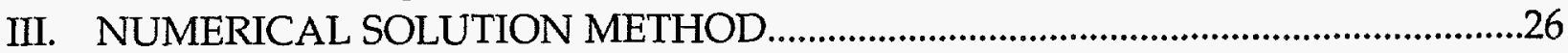

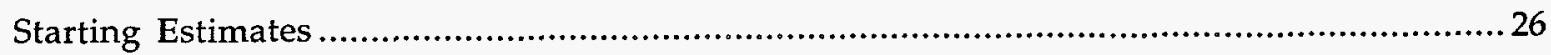

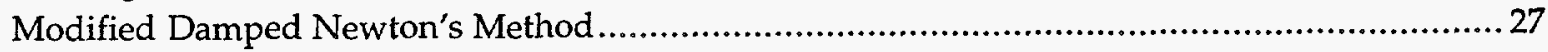

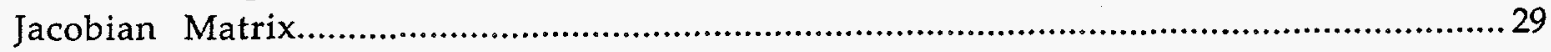

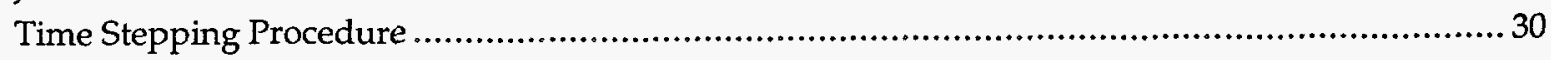

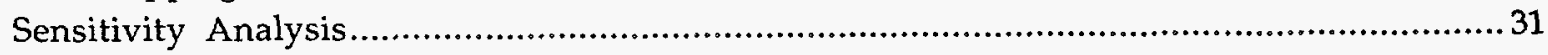

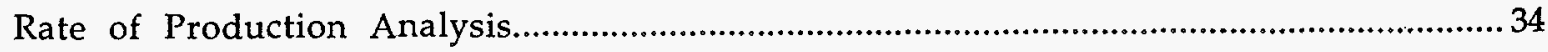

IV. PROGRAM STRUCTURE.............................................................................36

Structure of the Save, Recover, and Restart Files.................................................................. 38

The Driver Program for Aurora ...................................................................................... 43

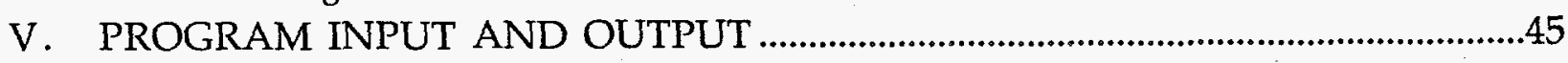

Keyword Descriptions............................................................................................ 46

VI. SAMPLE PROBLEM A: THERMAL CVD OF SI3N4 ........................................63

Chemkin Input for the Si3N4 Mechanism................................................................................66

Surface Chemkin Input for the Si3N4 Mechanism....................................................................68

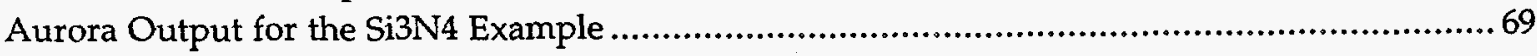

VII. SAMPLE PROBLEM B: LOW-PRESSURE CL2 PLASMA....................................82

Chemkin Input for the $\mathrm{Cl} 2$ Plasma Example..................................................................... 85

Surface Chemkin Input for the Cl2 Plasma Example...................................................... 87

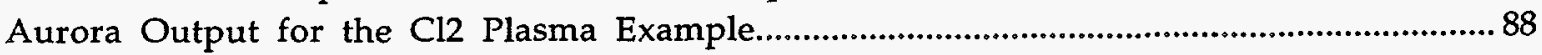

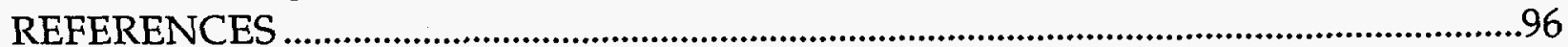

APPENDIX A. EXAMPLE SCRIPT FOR RUNNING CHEMKIN, SURFACE

CHEMKIN, AND AURORA .................................................................100 


\section{FIGURES}

Page

Figure 1. Schematic Representation of a Well Mixed Reactor ........................................... 11

Figure 2. Relationship between Aurora, Chemkin and Surface Chemkin Interpreters, and Associated Input and Output files......................................................................... 37

Figure 3. Lines of FORTRAN used to write the Save and Recover Files......................................39

Figure 4(a). Lines of FORTRAN used to read the Save, Recover and Restart Files. (a)

Reading in Chemistry Data................................................................................. 41

Figure 4(b). Lines of FORTRAN used to read the Save, Recover and Restart Files. (a)

Reading in Solution, Sensitivities, and Rates of Production............................................ 42

Figure 5. Example Driver Routine for Aurora...............................................................44 


\section{NOMENCLATURE}

\begin{tabular}{|c|c|c|}
\hline$\alpha_{k}$ & Activity of $k$ th bulk-phase species & $\begin{array}{l}\text { CGS Units } \\
\text { none }\end{array}$ \\
\hline$A$ & Total surface area of all materials in the reactor & $\mathrm{cm}^{2}$ \\
\hline$A_{m}$ & Surface area of the $m$ th material & $\mathrm{cm}^{2}$ \\
\hline$c_{k}$ & $\begin{array}{c}\text { Concentration of the } k \text { th species (gas-phase or bulk-phase) } \\
\text { (surface-phase) }\end{array}$ & $\begin{array}{l}\text { moles } / \mathrm{cm}^{3} \\
\text { moles } / \mathrm{cm}^{2}\end{array}$ \\
\hline$c_{p k}$ & Specific heat at constant pressure of the $k$ th species & $\mathrm{ergs} /(\mathrm{g} \mathrm{K})$ \\
\hline$G$ & Bulk growth rate & $\mathrm{cm} / \mathrm{sec}$ \\
\hline$\tilde{h}_{e}$ & Specific enthalpy of the electron upon creation in the gas-phase & $\mathrm{ergs} / \mathrm{g}$ \\
\hline$h_{k}$ & Specific enthalpy of the $k$ th species & $\mathrm{ergs} / \mathrm{g}$ \\
\hline$h_{t}$ & Heat transfer coefficient & $\mathrm{cal} / \mathrm{cm}^{2}-\mathrm{s}-\mathrm{K}$ \\
\hline$i$ & Reaction index or ionic species index & \\
\hline$I$ & Total number of reactions & \\
\hline$k$ & Species index & \\
\hline$K$ & Total number of species & \\
\hline$K_{b}(m)$ & Total number of bulk species on the $m$ th material & \\
\hline$K_{b}^{f}(n, m)$ & Index of the first bulk-phase species in phase $n$ of the $m$ th material & \\
\hline$K_{b}^{l}(n, m)$ & Index of the last bulk-phase species in phase $n$ of the $m$ th material & \\
\hline$K_{g}$ & Total number of gas-phase species & \\
\hline$K_{g}^{f}$ & Index of the first gas-phase species (always equal to 1) & \\
\hline$K_{g}^{l}$ & Index of the last gas-phase species (always equal to $K_{g}$ ) & \\
\hline$K_{s}(m)$ & Total number of surface species on the $m$ th material & \\
\hline$K_{s}^{f}(n, m)$ & Index of the first surface species in phase $n$ of the $m$ th material & \\
\hline$K_{s}^{l}(n, m)$ & Index of the last surface species in phase $n$ of the $m$ th material & \\
\hline$K_{\text {phase }}(m)$ & Vector containing the number of species in each phase & \\
\hline$m$ & Index for materials & \\
\hline$M$ & Number of materials in the surface reaction mechanism & \\
\hline$n$ & Index for phases & \\
\hline$N(m)$ & Total number of phases for the $m$ th material & \\
\hline$N_{b}(m)$ & Total number of bulk phases for the $m$ th material & \\
\hline$N_{b}^{f}(m)$ & Index of the first bulk phase for the $m$ th material & \\
\hline$N_{b}^{l}(m)$ & Index of the last bulk phase for the $m$ th material & \\
\hline$N_{g}$ & Number of gas phases (always equal to 1 ) & \\
\hline$N_{s}(m)$ & Total number of surface site types (phases) & \\
\hline
\end{tabular}




\begin{tabular}{|c|c|c|}
\hline$N_{s}^{f}(m)$ & Index of first surface phase & \\
\hline$N_{s}^{l}(m)$ & Index of last surface phase & \\
\hline$P$ & Pressure & dynes $/ \mathrm{cm}^{2}$ \\
\hline$P_{\text {atm }}$ & Pressure of one standard atmosphere & dynes $/ \mathrm{cm}^{2}$ \\
\hline$q_{i}$ & Rate of progress of the $i$ th reaction & moles $/\left(\mathrm{cm}^{2} \mathrm{sec}\right)$ \\
\hline$R$ & Universal gas constant & ergs/(mole K) \\
\hline$\dot{s}_{k}$ & Production rate of the $k$ th species from surface reactions & moles $/\left(\mathrm{cm}^{2} \mathrm{sec}\right)$ \\
\hline$t$ & Time & $\sec$ \\
\hline$T$ & Temperature of the bulk gas & $\mathrm{K}$ \\
\hline$T_{o}$ & Ambient temperature & $\mathrm{K}$ \\
\hline$T_{e}$ & Electron temperature & $\mathrm{K}$ \\
\hline$T_{\text {ion }}$ & Temperature of the ions & $\mathrm{K}$ \\
\hline$T_{\text {surf }}$ & Surface temperature & $\mathrm{K}$ \\
\hline$U$ & Velocity & $\mathrm{cm} / \mathrm{s}$ \\
\hline$V$ & Volume & $\mathrm{cm}^{3}$ \\
\hline$W_{k}$ & Molecular weight of $k \mathrm{th}$ species & $\mathrm{g} / \mathrm{mole}$ \\
\hline $\bar{W}$ & Mean molecular weight of a mixture & $\mathrm{g} / \mathrm{mole}$ \\
\hline$X_{k}$ & Mole fraction of the $k$ th species & none \\
\hline$Y_{k}$ & Mass fraction of the $k$ th species & none \\
\hline$Z_{k}(n, m)$ & Site fraction of the $k$ th species on site $n$ of the $m$ th material & none \\
\hline \multicolumn{3}{|l|}{ GREEK } \\
\hline$\beta_{i}$ & Temperature exponent in the rate constant of the $i$ th reaction & none \\
\hline$\rho$ & Mass density & $\mathrm{g} / \mathrm{cm}^{3}$ \\
\hline$\rho_{k}$ & Mass density of the $k$ th species & $\mathrm{g} / \mathrm{cm}^{3}$ \\
\hline$\rho_{n, m}$ & Site density of the $n$th bulk phase on the $m$ th material & $\mathrm{mole} / \mathrm{cm}^{2}$ \\
\hline$v_{k i}$ & Stoichiometric coefficient of species $k$ in reaction $i, v_{k i}=v_{k i}^{\prime \prime}-v_{k i}^{\prime}$ & \\
\hline$v_{k i}^{\prime}$ & Stoichiometric coefficient of the $k$ th reactant species in the $i$ th reaction & \\
\hline$v_{k i}^{\prime \prime}$ & Stoichiometric coefficient of the $k$ th product species in the $i$ th reaction & \\
\hline$\dot{\omega}_{k}$ & Production rate of the $k$ th species from gas-phase reactions & $\mathrm{mole} /\left(\mathrm{cm}^{2} \mathrm{sec}\right)$ \\
\hline$\sigma_{k}$ & Number of sites occupied by the $k$ th species & \\
\hline$\chi_{k}$ & Chemical symbol of the $k$ th species & \\
\hline
\end{tabular}




\section{AURORA: A FORTRAN PROGRAM FOR MODELING WELL STIRRED PLASMA AND THERMAL REACTORS WITH GAS AND SURFACE REACTIONS ${ }^{\dagger}$}

\section{INTRODUCTION}

Continuously stirred tank or well mixed reactor models have been in use for many years in the study of chemistry within a unit process for a variety of applications. For thermal (neutral) systems, perfectly stirred reactor (PSR) models are a common method for testing and developing chemical reaction mechanisms. ${ }^{4}$ Such reactor models are widely employed in combustion research. ${ }^{5-11,1}$ Moffat et al. ${ }^{1}$ included the capability to study detailed surface reaction mechanisms in a well stirred reactor simulation by incorporating general surface chemistry using the software, SURFACE CHEMKIN. ${ }^{12,13}$ This model has been applied to thermal chemical vapor deposition (CVD) systems. ${ }^{14}$ In the plasma community, global models have been used to predict average electron energies and electron densities for a variety of power-deposition systems. ${ }^{15-18}$ The model described here combines the generalized surface chemistry and neutral chemistry capabilities previously applied to thermal systems with the global plasma-modeling approach, through inclusion of an electron-energy or power balance and detailed electron-driven kinetics. The model builds on the Sandia perfectly stirred reactor software, SURFACE $P{ }^{1}$. This model includes the description of detailed surface kinetics and allows for the determination of surface-species site fractions, as well as bulk-phase deposition and etch rates. This approach allows investigation of systems with close coupling between gas-phase kinetics and surface kinetics. $\ddagger$

The contents of a well mixed or stirred reactor are assumed to be nearly spatially uniform due to high diffusion rates or forced turbulent mixing. In other words, the rate of conversion of reactants to products is controlled by chemical reaction rates and not by mixing processes. Thus the reactor is considered to be 'limited' by reaction kinetics. This is a very good assumption for the very low pressure, highly diffuse operating conditions of most plasma-etch reactors and some thermal CVD systems. An essential element of the stirred reactor model is the assumption that the reactor is sufficiently mixed to be described well by spatially averaged or bulk properties. For low-pressure processes (1 Torr and below), the dominance of species diffusion renders this assumption valid in many practical applications. The major advantage of the well stirred approximation lies in the relatively small computational demands of the mathematical model. Such a model allows investigators with only moderate computational resources to easily consider and analyze large, detailed chemical reaction mechanisms.

\footnotetext{
† Copyright $\odot$ 1996, Sandia Corporation. The U.S. Government retains a nonexclusive license in this software as prescribed in AL 88-1 and AL 91-7. Export of this program may require a license from the United States Government. ‡ Caution: AURORA works only with the newer CHEMKIN-III, not the original CHEMKIN or CHEMKIN-II packages; and works only with the newer SURFACE CHEMKIN-III, not the original SURFACE CHEMKIN package.
} 
In addition to fast mixing, the modeling of well-stirred reactors requires several assumptions. First, mass transport to the reactor walls is assumed to be infinitely fast. Therefore, the relative importance of surface reactions to gas-phase reactions is determined only by the surface-to-volume ratios of each material and the relative reaction rates (rather than by transport constraints). Second, the flow through the reactor must be characterized by a nominal residence time, which can be deduced from the flow rate and the reactor volume. The treatment of plasmas requires that power is deposited uniformly into the plasma bulk or, alternatively, that the electrons' diffusion and thermal conduction transport processes are fast relative to the rates of electron-driven chemical kinetics. In addition, we assume that the chemical reaction rate coefficients are independent of reactor conditions. This assumption may be violated in the case where the electron energy distribution function deviates significantly from Maxwellian conditions. ${ }^{16}$ However, modeling plasma systems in this manner still provides important insight into the reactor chemistry. ${ }^{16}, 17,19-21$ Finally, the characterization of a quasineutral bulk plasma depends on a thin-sheath approximation, where the sheath thickness is much smaller than the reactor-chamber dimensions.

The AURORA program includes an option to represent multiple stirred tank reactors that are connected in series. In such cases, we assume that the effluent of the preceding reactor is the input to the next stirred tank reactor. Each stirred tank reactor can have different temperatures, heating rates, volumes, and surface areas, for example.

Modeling well-stirred reactors requires solving systems of nonlinear algebraic equations. Solution of the equations in AURORA employs a modified Newton iteration method implemented in the numerical software, TWOPNT. ${ }^{22}$ This software was originally developed for application to premixed flame modeling, ${ }^{23}$ and has also been employed in boundary-layer chemically reacting flow problems. ${ }^{24,}$ 25 TWOPNT uses a hybrid Newton/time-integration procedure. Both Newton iteration ${ }^{26,6}$ and time integration ${ }^{27}$ have been used previously to solve well-stirred reactor problems. The advantage of the TWOPNT approach is that it combines the strengths of both methods. Consequently, the algorithm is both robust and efficient. In addition, the method facilitates rate-of-production and sensitivity analysis of computed results. Determination of chemical production rates and thermodynamic properties employs CHEMKIN III $^{2}$ and SURFACE CHEMKIN III $^{3}$ software packages.

This report begins with the description of the set of algebraic equations describing the well mixed reactor. This is followed by a detailed description of the numerical method and a method for calculating the first-order sensitivity coefficients of the mass fractions and temperature with respect to the rate constants. The report then includes a discussion of the code structure and a description of the user input keywords, as well as examples of CHEMKIN input files. Finally, we present two detailed sample problems. 


\section{WELL MIXED REACTOR EQUATIONS}

Conservation of mass, energy, and species describe a well mixed reactor including net generation of chemical species within the reactor volume, and net loss of species and mass to surfaces in the reactor. The stirred reactor consists of a chamber, which may or may not be thermally insulated, having inlet and outlet ducts. Figure 1 illustrates the conceptual representation of a generic reactor chamber. A steady flow of reactants is introduced through the inlet with a given species composition and temperature. The mass flow rate into the reactor, $\dot{m}^{*}$, may not be equal to the mass flow rate out of the reactor, $\dot{m}$, due to deposition on or etching of surface materials. In the AURORA model, the reactor pressure is specified, so that the conservation equations determine the volume outflow. Although Fig. 1 depicts a single surface in the reactor, an actual reactor may contain many different material surfaces, such as reactor walls, silicon wafer, substrate holder, etc. Each of these materials may have a different set of reaction kinetics associated with it. For this reason, we have included the capability of defining multiple surface materials that represent different fractions of the total surface area, with corresponding surface chemistry mechanisms.

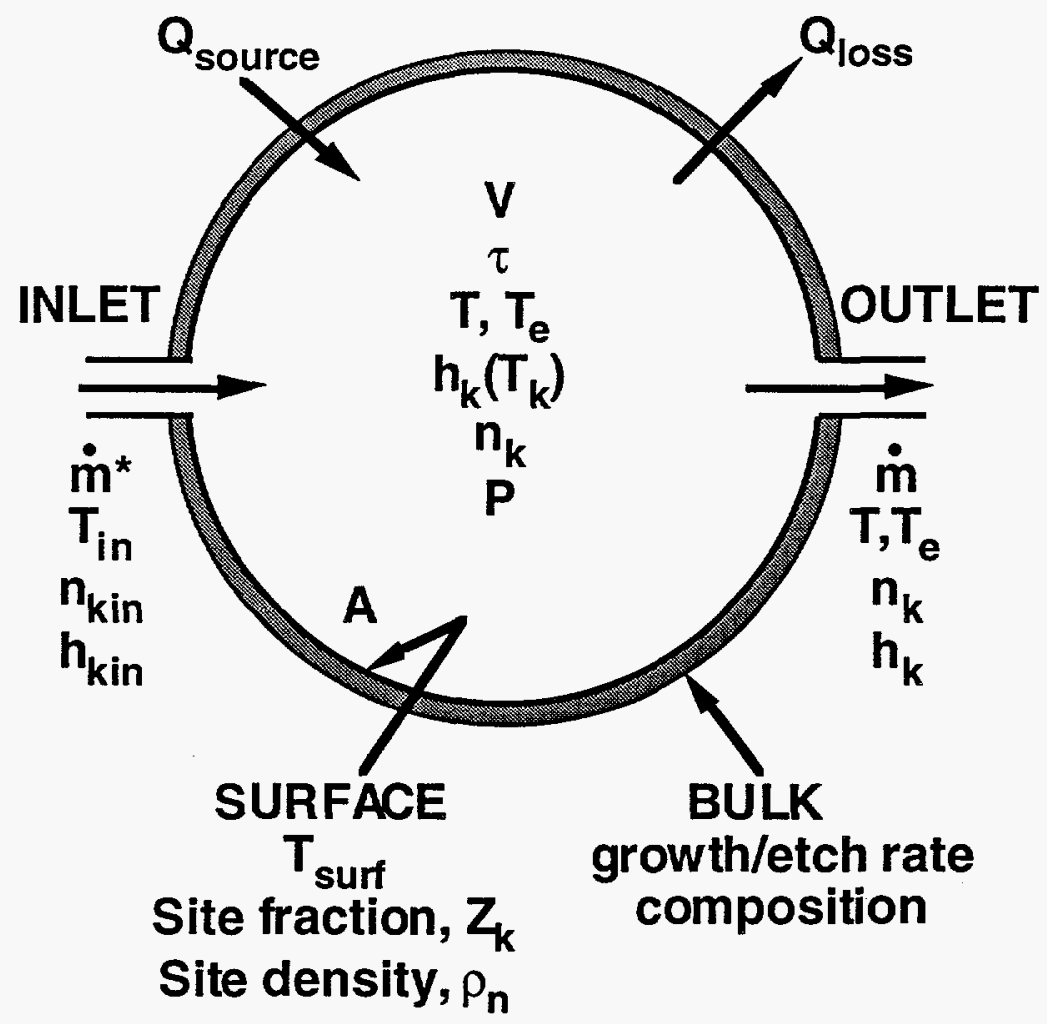

Figure 1. Schematic Representation of a Well Mixed Reactor 


\section{Mass Conservation and Gas-Phase Species Equations}

The AURORA model includes several unique capabilities for well mixed reactor modeling. First, the model allows for the description of plasma kinetics, where a system is characterized by more than one temperature (e.g. the electron temperature, the ion temperature, or a neutral gas temperature). In these cases, some reaction rates may depend on one temperature, while other reaction rates may depend on another. This capability is incorporated through the use of the multi-fluid CHEMKIN-III software package, ${ }^{2}$ and should not impact users who are only interested in systems that are in thermal equilibrium. Secondly, the model considers reaction kinetics on multiple surfaces within the reactor. Balances of surface species and bulk material species determine the surface state as well as net etch or deposition rates. This capability requires some hierarchy of information about gas-phase, surfacephase, and bulk-phase information. Only one gas phase is allowed, while more than one phase surface or bulk phase may be defined for each material. The details of this hierarchy are described in the SURFACE CHEMKIN-III manual. ${ }^{3}$

Even though we seek the solution to the steady-state equations, the computational algorithm often requires a partial solution of the related transient problem. Therefore, the transient conservation equations are presented here. We begin with global mass conservation in the reactor volume, where the time-rate of change of the mass in the reactor is equal to the difference between the mass flow in and the mass flow out, plus any material that is added to or subtracted from the surfaces within the chamber. This equations is stated as:

$$
\frac{d(\rho V)}{d t}=\dot{m}^{*}-\dot{m}+\sum_{m=1}^{M} A_{m} \sum_{k=1}^{K_{g}} \dot{s}_{k, m}
$$

Here $\rho$ is the mass density, $V$ is the reactor volume, $\dot{m}^{*}$ is the inlet mass flow rate, and $\dot{m}$ is the outlet mass flow rate. The outlet mass flow differs from the inlet mass flow when deposition or etching of materials within the reactor occurs, as represented by the last term on the right-hand side. In this term, $A_{m}$ is the surface area of the $m$ th material defined within the reactor, and $\dot{s}_{k, m}$ is the molar surface production rate of the $k$ th species on the $m$ th material per unit surface area. There are $K_{g}$ gasphase species and $M$ materials. This mass conservation equation can be rewritten to provide the relation between the outlet flow rate and the inlet flow rate as follows:

$$
\dot{m}=\dot{m}^{*}+\sum_{m=1}^{M} A_{m} \sum_{k=1}^{K_{g}} \dot{s}_{k, m}+\rho V\left(\frac{1}{\bar{T}} \frac{d \bar{T}}{d t}-\frac{1}{\bar{W}} \frac{d \bar{W}}{d t}\right)
$$

The time derivatives on the right-hand side result from the condition that the pressure and reactor volume are independent of time. $\bar{W}$ is the gas mixture's mean molecular weight, while $\bar{T}$ depicts the gas temperature, with a bar to indicate that this temperature actually represents a mixture-averaged temperature in multi-temperature plasma systems. In thermal systems, this is merely the gas 
temperature. In the AURORA solution, the two transient terms in Eq. (1) are neglected, since they will be small, and since we are primarily interested in the steady-state conditions.

The time-dependent equation for mass conservation of each gas-phase species, ignoring the implicit time dependence of $\rho$ through its dependence on the temperature and molecular weight, is

$$
\rho V \frac{d Y_{k}}{d t}=\dot{m}^{*}\left(Y_{k}^{*}-Y_{k}\right)+\dot{\omega}_{k} W_{k} V+\sum_{m=1}^{M} \dot{s}_{k, m} W_{k} A_{m}-Y_{k}\left(\sum_{m=1}^{M} A_{m} \sum_{k=1}^{K_{g}} \dot{s}_{k, m}\right) \quad k=1, \ldots, K_{g}
$$

or

$$
\frac{d Y_{k}}{d t}=\frac{1}{\tau}\left(Y_{k}^{*}-Y_{k}\right)+\frac{\dot{\omega}_{k} W_{k}}{\rho}+\frac{1}{\rho V} \sum_{m=1}^{M} A_{m}\left(\dot{s}_{k, m} W_{k}-Y_{k} \sum_{k=1}^{K_{g}} \dot{s}_{k, m}\right) \quad k=1, \ldots, K_{g}
$$

In these equations $Y_{k}$ is the mass fraction of the $k$ th species, $W_{k}$ is the molecular weight of the $k$ th species, and $\dot{\omega}_{k}$ is the molar rate of production of the $k$ th species by gas-phase chemical reaction per unit volume. The superscript * indicates inlet stream quantities.

The nominal residence time $\tau$ in the reactor is related to the reactor volume and the inlet mass flow rate as follows:

$$
\tau=\frac{\rho V}{\dot{m}^{*}}
$$

where the mass density $\rho$ is related to the pressure, gas temperature and electron temperature through the multi-fluid ideal gas equation of state (see, for example, the description of these relations in the CHEMKIN-III manual ${ }^{2}$ ). The residence time is often used as a characteristic parameter of the reactor, rather than the mass flow rate. In this case, $\dot{m}^{*}$ can be calculated from a specified residence time using Eq. (4).

\section{Surface Species Equations}

To determine surface-species site fractions, we begin with a conservation equation that balances the time rate of change of each surface species concentration with the net production rate of that surface species through chemical reactions at the surface. For each surface material there may be more than one surface phase; for example, $\mathrm{sp}^{2}$ and $\mathrm{sp}^{3}$ structures may be treated as two different phases in the surface mechanism. In addition, there may be several species defined for each phase representing, for example, open surface sites, physisorbed species, or chemisorbed species. Each surface phase contains an independent set of surface species; in other words, a given surface species exists in only one phase on 
only one material. The surface species conservation equation is applied to every species in each surface phase $n$ contained on each surface material $m$, as:

$$
\frac{d}{d t}\left(A_{m} c_{k} W_{k}\right)=A_{m} W_{k} \dot{s}_{k} \quad k=K_{s}^{f}(m), \ldots, K_{s}^{l}(m) ; m=1, \ldots, M
$$

Here $A_{m}$ is the surface area of the $m$ th material in the reactor and $c_{k}$ is the molar concentration of the $k$ th surface species $\left(\right.$ moles $/ \mathrm{cm}^{2}$ ).

Within the AURORA code, we use a strict ordering of species, species types, and surface materials that allows very general descriptions of the chemistry between species of all types in the reactor. The subscript $k$ is then used for all species, whether gas-phase, surface-phase, or bulk-phase. For this reason, in Eq. (5) and subsequent equations, we introduce somewhat complicated indices for the range of surface species. Specifically, $K_{s}^{f}\left(N_{s}^{f}(m), m\right)$ refers to the first surface-phase species in the first surface phase of the material $m$, while $K_{s}^{l}\left(N_{s}^{l}(m), m\right)$ refers to the last surface-phase species of the last surface phase of the $m$ th material. The total number of surface materials is $M$, with the total number of surface phases on each material given by $N_{s}(m)$ and the total number of bulk phases by $N_{b}(m)$. The surface phase index range for a material is given by $N_{s}^{f}(m) \leq n \leq N_{s}^{l}(m)$. The first phase defined in the system is always the gas-phase, with $n=1$, such that $N_{s}^{f}(1)=2$ and $K_{s}^{f}(2,1)=K_{g}+1$ in all cases where surface species are defined. In each phase, $n$, there are $K_{\text {phase }}(n, m)$ species, whose indices are in the range, $K_{s}^{f}(n, m) \leq k \leq K_{s}^{l}(n, m)$. When the species index range includes all surface species, as in Eq. 5, we introduce a shorthand notation, where $K_{s}^{f}(m)=K_{s}^{f}\left(N_{s}^{f}(m), m\right)$ and $K_{s}^{l}(m)=K_{s}^{l}\left(N_{s}^{l}(m), m\right)$. Although this nomenclature is somewhat confusing on first glance, it need not unduly concern the user; all of this formalism is strictly maintained internally in the AURORA code structure through use of SURFACE CHEMKIN routines. For further insight into this nomenclature, see the SURFACE CHEMKIN user's manual. ${ }^{3}$

The molar concentration of a surface species is related to the total site density of a surface phase, $\rho_{n, m}$, to the site fraction of the $k$ th surface species, and to the species coverage factor $\sigma_{k}$, as follows:

$$
c_{k}=\frac{\rho_{n, m} Z_{k}}{\sigma_{k}}
$$

Assuming the surface areas of each material are constant, substituting Eq. (6) into Eq. (5), and expanding the derivatives, we obtain:

$$
\frac{d Z_{k}}{d t}=\sigma_{k} \frac{\dot{s}_{k, m}}{\rho_{n, m}}-\frac{Z_{k}}{\rho_{n, m}} \frac{d \rho_{n, m}}{d t} \quad k=K_{s}^{f}(m), \ldots, K_{s}^{l}(m) ; m=1, \ldots, M
$$

The net change in surface-phase site densities is defined as: 


$$
\sum_{k=K_{s}^{f}(n, m)}^{K_{s}^{l}(n, m)} \dot{s}_{k, m}=\frac{d \rho_{n, m}}{d t}
$$

$$
n=N_{s}^{f}(m), \ldots, N_{s}^{l}(m) ; m=1, \ldots, M
$$

Equation (8) is the surface site conservation equation that must hold true for each surface phase in each material. Here we note that, for the steady-state case, applying Eq. (7) to all surface species will lead to a singular Jacobian matrix. To obtain a well posed system of equations, one must introduce the additional requirement that all surface site fractions, $Z_{k}$, sum to one:

$$
1=\sum_{k=K_{s}^{f}(n, m)}^{K_{s}^{l}(n, m)} Z_{k}
$$

Eq. (9) may then be solved directly in place of one of the surface-species balances in Eq. (7). However, this approach results in the numerical round-off error in the calculation of the all of the $\dot{s}_{k}$ 's to be assigned to one equation (Eq. 9). This may cause problems in the case when that surface-site fraction is small. Instead, we chose to apportion the additional constraint represented by Eq (9) over all of the equations represented by Eq. (7) for the surface phase $n$ according to the size of the surface site fraction, as follows:

$$
\frac{d Z_{k}}{d t}=\sigma_{k} \frac{\dot{s}_{k, m}}{\rho_{n, m}}-\frac{Z_{k}}{\rho_{n, m}} \frac{d \rho_{n, m}}{d t}+\frac{Z_{k}}{\tau}\left(1-\sum_{l=K_{s}^{f}(n, m)}^{K_{s}^{l}(n, m)}\right) \quad k=K_{s}^{f}(m), \ldots, K_{s}^{l}(m) ; m=1, \ldots, M
$$

Note that the time constant for the second term on the right-hand-side of Eq. (10) is arbitrary, and we have chosen $\tau$ because it is dimensionally correct and physically significant for the well mixed reactor model. If Eq. (10) is summed over all surface species in phase $n$ and combined with Eq. (8), then the following relation results:

$$
\frac{d\left(1-\sum_{l=K_{s}^{f}(n, m)}^{l=K_{l}^{l}(n, m)} Z_{l}\right)}{d t}=-\frac{\left(1-\sum_{l=K_{s}^{f}(n, m)}^{l=K_{l}^{l}(n, m)} Z_{l}\right)}{\rho_{n, m}} \frac{d \rho_{n, m}}{d t}-\frac{\left(1-\sum_{l=K_{s}^{f}(n, m)}^{l=K_{l}^{l}(n, m)} Z_{l}\right)}{\tau},
$$

which is equivalent to the sum of the $Z_{k}$ 's being equal to one, with a false transient.

\section{Bulk Species Equations During Deposition}

AURORA allows for the deposition and etching of bulk phases of materials in the reactor, although we do not account for changes in reactor volume or surface area with time due to etch or deposition processes. When there is only a single bulk species in a bulk phase, the bulk species mole fraction is trivially defined as one. When more than one bulk species exist in a bulk phase, the composition of the bulk phase may change with time, requiring solution of the bulk species mole fraction. For bulk phases that are being deposited, the mole fractions of the bulk-phase species are 
assumed to be initially unknown and are determined through time-dependent species balance equations for each bulk species:

$$
\frac{d}{d t}\left(A_{m} L_{n, m} X_{k}^{b} C_{n, m}^{b} W_{k}\right)=A_{m} W_{k} \dot{s}_{k, m}
$$

or

$$
A_{m} L_{n, m} C_{n, m}^{b} W_{k} \frac{d}{d t}\left(X_{k}^{b}\right)+A_{m} W_{k} X_{k}^{b} \frac{d}{d t}\left(L_{n, m} C_{n, m}^{b}\right)=A_{m} W_{k} \dot{s}_{k, m}
$$

Here $X_{k}^{b}$ is the bulk-species mole fraction of the $k$ th species in phase $n$ of the $m$ th material. $L_{n, m}$ is the film thickness for the $n$th bulk phase of the mth material. $C_{n, m}^{b}$ is the average molar concentration of the species in the $n$th bulk phase of the $m$ th material.

The molar growth rate of the bulk phase $n$, can be written as

$$
\frac{d\left(A_{m} L_{n, m} C_{n, m}^{b}\right)}{d t}=\sum_{k=K_{b}^{f}(n, m)}^{K_{b}^{l}(n, m)} A_{n} \dot{s}_{k, m}
$$

while the bulk mass density is defined as

$$
\rho_{n, m}^{b}=C_{n, m}^{b} \bar{W}_{n, m}^{b}, \quad n=N_{b}^{f}(m), \ldots, N_{b}^{l}(m) ; m=1, \ldots, M
$$

where $\bar{W}_{n, m}^{b}$ is the mean molecular weight of the bulk phase. Substituting Eqs. (14-15) into Eq. (13) and rearranging gives the following relation:

$$
A_{m} L_{n, m} \rho_{n, m}^{b} \frac{W_{k}}{\bar{W}_{n, m}^{b}} \frac{d X_{k}^{b}}{d t}=A_{m} W_{k} \dot{s}_{k_{s} m}-A_{m} W_{k} X_{k}^{b} \sum_{k=K_{b}^{f}(n, m)}^{K_{b}^{l}(n, m)} \dot{s}_{k, m},
$$

which simplifies to:

$$
\frac{d X_{k}^{b}}{d t}=\frac{\bar{W}_{n, m}^{b}}{L_{n, m} \rho_{n, m}^{b}}\left[\dot{s}_{k, m}-X_{k}^{b} \sum_{k=K_{b}^{f}(n, m)}^{K_{b, m}^{l}(n, m)} \dot{s}_{k, m}\right]
$$

We define the film-thickness length scale, $L_{n, m}$, somewhat arbitrarily as the linear growth rate of the bulk phase multiplied by the reactor residence time, i.e.:

$$
L_{n, m}=\tau \frac{\bar{W}_{n, m}^{b}}{\rho_{n, m}^{b}} \sum_{k=K_{b}^{f}(n, m)}^{K_{b}^{l}(n, m)} \dot{s}_{k, m}
$$

For deposition phases, then, Eqs. (17-18) provide a false transient equation that is employed in AURORA for the bulk species: 


$$
\frac{d X_{k}^{b}}{d t}=\frac{1}{\tau}\left[\frac{\dot{s}_{k, m}}{\sum_{\substack{K_{b}^{l}(n, m) \\ k=K_{b}^{f}(n, m)}} \dot{s}_{k, m}}-X_{k}^{b}\right]
$$

Note that $L_{n, m}$ does not appear in Eq. (19); AURORA makes no assumption about the total amount of bulk phase deposits, other than that their total amount is small compared to the total volume of the reactor. Eq. (19) is only used when the right-hand side is greater than zero; i.e. when there is net deposition of the bulk species. Under etching conditions, the bulk species equations must be treated differently.

\section{Bulk Species Equations During Etch}

When surface reactions that result in etching of a bulk phase are included in the surface reaction mechanism, the user must supply the initial composition of that phase (unless there is only one bulk species in the phase). The time-dependent equation for the bulk-phase species for phases that etch is then:

$$
\frac{d X_{k}^{b}}{d t}=\frac{X_{k}^{b 0}-X_{k}^{b}}{\tau}+\frac{X_{k}^{b}}{\tau}\left[1-\sum_{l=K_{b}^{f}(n, m)}^{K_{b}^{l}(n, m)} X_{l}^{b}\right]
$$

where $X_{k}^{b 0}$ is the user-supplied initial estimate for the mole fraction of species $k$ in bulk phase $n$, normalized so that their sum over all bulk-phase species is equal to one.

\section{Non-Constant Surface-Phase Site Densities}

Although surface site densities are very often considered physical constants associated with a particular surface structure, there are some cases when the surface site density may change with time. SURFACE CHEMKIN allows the inclusion of reactions that do not conserve surface sites, if this is a desired feature of the problem. In such cases, the surface site density $\rho_{n, m}$, for every phase $n$ whose total number of sites may not be conserved, becomes an additional solution unknown. The total number of surface phases on each material $m$, for which this condition may hold, is defined as equal to the variable $N_{\rho}(m)$. For each material $m$, there are $N_{\rho}(m)$ additional equations to be solved, corresponding to $N_{\rho}(m)$ additional unknowns. The equation for the time derivative of surface site densities is given by 


$$
\frac{d \rho_{n, m}}{d t}=\sum_{k=K_{s}^{f}(n, m)}^{l} \sigma_{k} \dot{s}_{k, m}
$$

$$
n=N_{\rho}^{f}(m), \ldots, N_{\rho}^{l}(m)
$$

\section{Gas Energy Equation}

For the gas temperature, AURORA either accepts a fixed temperature value specified by the user (TGIV keyword), or solves an energy balance in the reactor (ENRG keyword). The energy balance is determined by considering a control volume that includes the reactor, the reactor walls, and any deposited material therein. The following equation for the total internal energy of the reactor system is then

$$
\frac{d U_{\text {sys }}}{d t}=\dot{m}^{*} \sum_{k=1}^{K_{g}} Y_{k}^{*} h_{k}^{*}-\dot{m} \sum_{k=1}^{K_{g}} Y_{k} h_{k}-Q_{\text {loss }}+Q_{\text {source }}
$$

The total internal energy $U_{\text {sys }}$ consists of the internal energy of the gas, surface phases, deposited or etched solid phases, and walls. $Q_{\text {loss }}$ is the net heat flux directed out of the reactor. $Q_{\text {loss }}$ can either be specified directly as a constant (QLOS keyword) or can be specified in terms of a constant heat transfer coefficient, $h_{t}$, and ambient temperature, $T_{o}$ (HTRN keyword), as follows:

$$
Q_{\text {loss }}=A h_{t}\left(T-T_{o}\right)
$$

$Q_{\text {source }}$ refers to energy deposited into the gas in the reactor. For plasma systems, this can represent the power deposited through Joule heating into the plasma by acceleration of charged species along electric-fields. This term will be discussed in more detail in the description of the electron energy equation below.

With the assumption of constant pressure and constant volume in the reactor, the time derivative of the internal energy can be equated with the time derivative of the enthalpy, using:

$$
\begin{aligned}
U_{\text {sys }} & =U_{\text {gas }}+\sum_{m=1}^{M} \sum_{n=N_{s}^{f}}^{N_{b}^{l}} U_{n}(m)+U_{\text {walls }} \\
& =H_{\text {gas }}+\sum_{m=1}^{M} \sum_{n=N_{s}^{f}}^{N_{b}^{l}}\left(H_{n}(m)-p V_{n}\right)+H_{\text {walls }}-p V_{\text {walls }}
\end{aligned}
$$

The left-hand side of Eq. (21) then becomes:

$$
\frac{d U_{\text {sys }}}{\mathrm{dt}}=\frac{d H_{\mathrm{gas}}}{\mathrm{dt}}+\sum_{m=1}^{M} \sum_{n=N_{s}^{f}}^{N_{b}^{l}} \frac{d H_{n}(m)}{d t}+\frac{d H_{\mathrm{walls}}}{d t}
$$


We neglect the last term on the right-hand-side that represents the heat capacity contribution from the walls. Expansion of the gas-phase contribution in Eq. (25) yields:

$$
\frac{d H_{\mathrm{gas}}}{d t}=\frac{d(\rho V \bar{h})}{d t}=\rho V\left(\sum_{k=1}^{K_{g}} Y_{k} c_{p k} \frac{d T_{k}}{d t}\right)+\rho V\left(\sum_{k=1}^{K_{g}} h_{k} \frac{d Y_{k}}{d t}\right)+V \sum_{k=1}^{K_{g}} Y_{k} h_{k} \frac{d \rho}{d t},
$$

where $\bar{h}$ is the specific enthalpy of the gas mixture equal to the sum of the product of the species mass fraction and the pure species specific enthalpy. Note that in a multi-temperature system, the species enthalpies are evaluated at the species temperature $T_{k}$ which may differ from the mean gas temperature. In thermal systems, all $T_{k}$ equal $T$, the gas temperature. $c_{p k}$ represents the species specific heat capacity at constant pressure. Expansion of the bulk and surface contributions to Eq. (25) yields:

$$
\begin{aligned}
& \sum_{m=1}^{M} \sum_{n=N_{b}^{f}(m)}^{N_{b}^{l}(m)} \frac{d H_{n}(m)}{d t}=\sum_{m=1}^{M} \sum_{n=N_{b}^{f}(m)}^{N_{b}^{l}(m)}\left\{h_{n}(m) \bar{W}_{n, m}^{b} \frac{d}{d t}\left(A_{m} L_{n, m} C_{n, m}^{b}\right)+A_{m} L_{n, m} C_{n, m}^{b} \frac{d}{d t}\left(h_{n}(m) \bar{W}_{n, m}^{b}\right)\right\} \\
& \quad+\sum_{m=1}^{M} \sum_{n=N_{s}^{f}(m)}^{N_{s}^{l}(m)} \sum_{k=K_{s}^{f}(n, m)}^{K_{s}^{l}(n, m)}\left\{h_{k} \frac{d\left(A_{m} c_{k} W_{k}\right)}{d t}+A_{m} c_{k} W_{k} \frac{d h_{k}}{d t}\right\}
\end{aligned}
$$

If we neglect the time dependence of the bulk- and surface-phase species enthalpies and molecular weights, and make use of Eq. (12), Eq. (27) is greatly simplified to:

$$
\sum_{m=1}^{M} \sum_{n=N_{s}^{f}(m)}^{N_{b}^{l}(m)} \frac{d H_{n}(m)}{d t}=\sum_{m=1}^{M} \sum_{k=K_{s}^{f}(m)}^{K_{b}^{l}(m)} A_{m} \dot{s}_{k, m} W_{k} h_{k}
$$

Combining Eqs. (2-3), (22), (25), (26), and (28) gives the transient energy equation for solving the gas temperature, as follows:

$$
\begin{aligned}
\rho V\left(\bar{c}_{p}\left(1-Y_{e}\right) \frac{d T}{d t}+Y_{e} c_{p e} \frac{d T_{e}}{d t}\right) \\
=\dot{m}^{*} \sum_{k=1}^{K_{g}} Y_{k}^{*}\left(h_{k}^{*}-h_{k}\right)-V \sum_{k=1}^{K_{g}} h_{k} \dot{\omega}_{k} W_{k}-\sum_{m=1}^{M} A_{m} \sum_{k=1}^{K_{g}} h_{k} \dot{s}_{k, m} W_{k}-Q_{\text {loss }}-Q_{\text {source }}
\end{aligned}
$$

Here we define $\bar{c}_{p}$ as the mean gas specific heat excluding the contribution of the electrons, since we assume that the electron temperature may be significantly different from the gas temperature. All other species are assumed to be in thermal equilibrium at the gas temperature $T$. In other words,

$$
\bar{c}_{p} \equiv \sum_{k=1 ; k \neq e}^{K_{g}} Y_{k} c_{p k}
$$


where the subscript, $e$, indicates the electron species. When no electrons are present Eq. (29) reverts back to thermal-equilibrium case and the mean specific heat is merely the mass-averaged value of all species components. The actual form of the gas energy equation solved in AURORA is the result of subtracting the electron energy equation from Eq. (29). This form is presented after the introduction of the electron energy equation below.

\section{Electron Energy Equation for Plasma Systems}

The transient form of the electron energy balance equates the time-rate-of-change of the electron swarm's internal energy, $u_{e}$, to the net flow of electron enthalpy into and out of the reactor, accounting for net chemical production rates, surface losses, collisional losses, and power deposition from externally applied electromagnetic fields. This balance is stated as:

$$
\frac{d}{d t}\left(\rho_{e} V u_{e}\right)=\dot{m}^{*} Y_{e}^{*} h_{e}^{*}-\dot{m} Y_{e} h_{e}+\dot{\omega}_{e} W_{e} \tilde{h}_{e} V+\sum_{m=1}^{M} \dot{s}_{e, m} W_{e} h_{e} A_{m}-Q_{\mathrm{loss}}^{\text {elas }}-Q_{\mathrm{loss}}^{\text {inel }}+Q_{\text {source }}^{\prime}
$$

where $\rho_{e}$ is the electron mass density (equal to the product of the electron number density and the electron mass). $\tilde{h}_{e}$ refers to the electron enthalpy of newly created electrons in the gas-phase; when electrons are formed from the ionization of a relatively cold neutral, the electron is assumed to originate close to the neutral temperature. The energy required to thermalize new electrons is therefore taken into account. At the surface, electron losses are assumed to dominate electron emission, so that no equivalent term is included to account for new electrons coming off the surface with thermal energies equal to the surface temperature. The electron enthalpy loss at the surface is therefore calculated from the net production rate of electrons due to surface reactions on each material, $\dot{s}_{e, m}$, and the electron enthalpy, $h_{e}$. The second- and third-to-last terms on the right-hand-side refer to the collision energy lost by the electrons both from elastic, momentum-transfer collisions, and from inelastic collisional processes. The inelastic collisions may include both excitation reactions, as well as chemical reactions resulting from electron-impact collisions. The source term $Q_{\text {source }}^{\prime}$ differs from $Q_{\text {source }}$ in Eq. (29) in that it represents only that power deposited to the electrons, rather than to the plasma as a whole. In particular, some of the deposited power may contribute to heating of ions in the plasma bulk, or accelerating ions through the plasma sheath. We therefore define the electron-energy source term as:

$$
Q_{\text {source }}^{\prime}=Q_{\text {source }}-Q_{\text {ions }}^{\text {sheath }}-Q_{\text {ions }}^{\text {plasma }}
$$

The internal energy of the electron and electron specific heats are defined by:

$$
u_{e}=h_{e}-\frac{P_{e}}{\rho_{e}}=\frac{5}{2} \frac{R}{W_{e}} T_{e}-\frac{R T_{e}}{W_{e}}=\frac{3}{2} \frac{R}{W_{e}} T_{e} ; c_{p e}=\frac{5}{2} \frac{R}{W_{e}} ; c_{v e}=\frac{3}{2} \frac{R}{W_{e}}
$$


If we assume $\tilde{h}_{e}=(5 / 2) R T / W_{e}$, substitute Eqs. (1), (32), and (33) into Eq. (31), and subtract Eq. (2) multiplied by $c_{p e} T_{e}$, we arrive at:

$$
\rho V\left(Y_{e} c_{v e} \frac{d T_{e}}{d t}-\frac{R}{W_{e}} T_{e} \frac{d Y_{e}}{d t}\right)=\dot{m}^{*} Y_{e}^{*} c_{p e}\left(T_{e}^{*}-T_{e}\right)+\dot{\omega}_{e} W_{e} V c_{p e}\left(T-T_{e}\right)-Q_{\text {loss }}^{\text {elas }}-Q_{\text {loss }}^{\text {inel }}+Q_{\text {source }}^{\prime}
$$

Here the second term on the right-hand-side represents the thermalization energy required for newly created electrons. The loss and source terms in Eqs. (34) and (32) are defined as follows:

$$
\begin{aligned}
& Q_{\text {loss }}^{\text {elas }}=3 V R \rho_{e}\left(T_{e}-T\right) \sum_{k=1 ; k \neq e}^{K_{g}} \frac{W_{e}}{W_{k}} v_{e k}, \\
& Q_{\text {loss }}^{\text {inel }}=V \sum_{r=1}^{I_{e i}} \Delta H_{r} q_{r}+Q_{\text {other }}\left(T_{e}\right), \\
& Q_{\text {ions }}^{\text {sheath }}=\sum_{m=1}^{M} \sum_{k=1}^{K_{g}} \dot{s}_{i, m} \varepsilon_{i} A_{m},
\end{aligned}
$$

and

$$
Q_{\text {ions }}^{\text {plasma }}=\sum_{i=1}^{K_{i}} \dot{\omega}_{i}\left(h_{i}\left(T_{\text {ion }}\right)-h_{i}(T)\right) V
$$

In Eq. (35), $v_{e k}$ is the momentum-transfer collision frequency between the electrons and the $k$ th heavy species. AURORA calculates the momentum-transfer collision frequencies from momentum-transfer collision cross-sections specified with the input keywords XSEK and XSDF. The first term in Eq. (36) represents the summation of electron energy loss per electron-impact reactions as specified in the CHEMKIN input file. The total number of electron-impact (i.e. electron-temperature dependent) reactions is $I_{e i}, q_{r}$ is the net rate of progress of the $r$ th reaction, and $\Delta H_{r}$ is the net enthalpy change of the reaction. $\Delta H_{r}$ can be determined from species' thermochemistry as available in the CHEMKIN thermodynamic data, or can be input directly through use of the CHEMKIN reaction auxiliary keyword EXCI (see the CHEMKIN-III user's manual. ${ }^{2}$ The second term on the right-hand side of Eq. (36) represents other loss terms that the user may choose to include separately from the CHEMKIN reaction descriptions (using the AURORA keyword QLSE). In Eq. (37), $\varepsilon_{i}$ is the energy gained by an ion when traversing the sheath, while $K_{i}$ is the total number of ionic species. $\varepsilon_{i}$ may be supplied through one of several options: direct specification (IONE keyword), calculation from the electron temperature and a user-specified multiplication factor (ELSH keyword), or through solution of an analytical radiofrequency sheath model within AURORA with user-specified rf-bias conditions (RFSH and related keywords). The rf sheath model is described in more detail below. In Eq. (38), we introduce an ion temperature $T_{\text {ion }}$ to capture the source energy that is deposited into the ions, although we are not solving an ion energy equation explicitly. The ion temperature is specified by the user (TION keyword) and assumed to be constant. 


\section{Gas Energy Equation Adjusted for Plasma Systems}

The actual gas energy equation solved in AURORA results from subtracting Eq. (34) from Eq. (29). This approach keeps only those terms that directly affect the gas temperature in the gas-energy equation. The resulting gas-energy equation is then:

$$
\begin{aligned}
\rho V\left(\bar{c}_{p}\left(1-Y_{e}\right) \frac{d T}{d t}+\frac{R}{W_{e}} \frac{d\left(Y_{e} T_{e}\right)}{d t}\right) \\
=\dot{m}^{*} \sum_{k=1 ; k \neq e}^{K_{g}} Y_{k}^{*}\left(h_{k}^{*}-h_{k}\right)-V \sum_{k=1 ; k \neq e}^{K_{g}} h_{k} \dot{\omega}_{k} W_{k}-\dot{\omega}_{e} W_{e} V \tilde{h}_{e} \\
\quad-\sum_{m=1}^{M} A_{m} \sum_{k=1 ; k \neq e}^{K_{g}} h_{k} \dot{s}_{k, m} W_{k}-Q_{\text {loss }}+Q_{\text {loss }}^{\text {elas }}+Q_{\text {loss }}^{\text {inel }}+Q_{\text {ions }}^{\text {plasma }}+Q_{\text {ions }}^{\text {sheath }} .
\end{aligned}
$$

\section{Application of the Bohm Condition for Ion Fluxes to Surfaces}

Often, when modeling very low-pressure plasmas, it is reasonable to constrain the ion flux to a surface according to the Bohm criterion. This condition maintains that the maximum net flux of a particular ion to a surface is equal to the product of the ion density and the Bohm velocity, which is defined as:

$$
U_{\mathrm{Bohm}}=\left(\frac{R T_{e}}{W_{i}}\right)^{1 / 2}
$$

For an electronegative gas, this expression must be modified to account for the presence of negative ions and their effect on the plasma sheath behavior. In the limit of a purely electronegative gas, the ion flux to a surface would be limited by the thermal speed of the ion. To accommodate a large range of conditions, then, we use the correction to the Bohm velocity derived by Braithewaite and Allen, ${ }^{28}$ as follows:

$$
U_{\mathrm{Bohm}, \bmod }=\left(\frac{R T_{e}}{W_{i}}\right)^{1 / 2}\left[\left(\frac{c_{n, \mathrm{tot}}}{\mathrm{c}_{\mathrm{e}}+c_{n, \mathrm{tot}}}\right)\left(\frac{T_{i}}{T_{e}}\right)^{1 / 2}+\left(\frac{\mathrm{c}_{\mathrm{e}}}{\mathrm{c}_{\mathrm{e}}+c_{n, \mathrm{tot}}}\right)\right],
$$

where $c_{n \text {,tot }}$ is the sum over all negative ions of the product of the ion species ${ }^{\prime}$ concentration and its integer electronic charge.

AURORA allows the user to specify this constraint in one of two ways. The first way is to use the AURORA keyword BOHM. This keyword includes the input of a correction factor $\xi$ to the above Bohm velocity. When this option is included, the production rate of each ion by each surface reaction will be scaled, such that the net production rate of the ion on each material is given by 


$$
\dot{s}_{i, m}=\dot{s}_{i, \mathrm{Bohm}}=\xi_{c_{i}} U_{\mathrm{Bohm}, \mathrm{mod}}=\xi c_{i}\left(\frac{R T_{e}}{W_{i}}\right)^{1 / 2}\left[\left(\frac{c_{n, \text { tot }}}{c_{\mathrm{e}}+c_{n, \mathrm{tot}}}\right)\left(\frac{T_{i}}{T_{e}}\right)^{1 / 2}+\left(\frac{c_{e}}{c_{\mathrm{e}}+c_{n, \mathrm{tot}}}\right)\right]
$$

The second way to apply the modified Bohm criterion, is to use the SURFACE CHEMKIN BOHM auxiliary keyword. In this case, the individual reaction for which the auxiliary BOHM keyword is included, will have a rate of progress calculated in the SURFACE CHEMKIN routines as

$$
q=\xi_{c_{i}} U_{\mathrm{Bohm}}=\xi c_{i}\left(\frac{R T_{e}}{W_{i}}\right)^{1 / 2}
$$

where, in this case, the correction factor $\xi$ is the first reaction-rate coefficient specified on the reaction line in the SURFACE CHEMKIN input file. This rate-of-progress of the BOHM reaction will then be modified within AURORA to account for the presence of any negative ions in the plasma; i.e. each reaction with a BOHM auxiliary keyword ultimately has a rate of progress in AURORA defined as:

$$
q_{\bmod }=\xi c_{i}\left(\frac{R T_{e}}{W_{i}}\right)^{1 / 2}\left[\left(\frac{c_{n, \text { tot }}}{\mathrm{c}_{\mathrm{e}}+c_{n, \text { tot }}}\right)\left(\frac{T_{i}}{T_{e}}\right)^{1 / 2}+\left(\frac{\mathrm{c}_{\mathrm{e}}}{\mathrm{c}_{\mathrm{e}}+c_{n, \text { tot }}}\right)\right]
$$

The main difference between these two approaches arises when an ion participates in more than one surface reaction subject to the Bohm criterion. In the first approach, the net ion flux to the surface will be automatically scaled to the Bohm-limiting flux modified for electronegative gases and the userdefined correction factor $\xi$. In the latter approach, each reaction will be subject to the Bohm limit independently. In that case, it is up to the user to make sure that the reaction coefficients $\xi$ add up to the desired overall correction factor, for all the reactions involving a particular ion. In AURORA, the overall correction factor is often used to account for spatial variations in ion density or transport limitations in the reactor being modeled. For example, the correction factor may be set equal to an estimation of the ratio of the ion density at the sheath edge to the ion density in the bulk of the plasma. ${ }^{17,21,20}$

\section{Summary of Solution Variables}

The total number of species defined in the systems is

$$
K=K_{g}+\sum_{m=1}^{M}\left[K_{s}(m)+K_{b}(m)\right]
$$

where $K_{g}$ is the number of gas-phase species; $K_{s}(m)$ and $K_{b}(m)$ is the number of surface- and bulk-phase species on the $m$ th material; and $M$ is the total number of different reacting materials defined in the reactor. The maximum number of unknowns in the problem, $J$, is therefore given by 


$$
J=2+K+\sum_{m=1}^{M} N_{\rho}(m)
$$

where, in addition to species fractions and surface-phase densities, we may be solving for the gas temperature and the electron temperature. The equation corresponding to gas-phase species is Eq. (3). The equation corresponding to surface-phase site fractions is Eq. (10), while the equation for bulk species is either Eq. (19) or Eq. (20), depending on whether or not the bulk species may be etched. The equations corresponding to site densities, in the case when non-site-conserving reactions are included in the surface mechanism, is Eq. (21). The gas temperature is determined by Eq. (39) and the electron energy equation is Eq. (34). AURORA solves for the steady-state versions of the above equations.

\section{Treatment of Activities for Bulk Species}

The net gas-phase chemical production rate of each species, $\dot{\omega}_{k}$, results from the competition between all the chemical reactions involving that species. For reactions stated as reversible in the CHEMKIN and SURFACE CHEMKIN input files, the reverse rate parameters can be determined from the forward rate coefficients and the thermodynamic properties of the species involved in the reaction, via the law of mass action. In these cases, SURFACE CHEMKIN requires activities to be defined for bulk-phase species, in order to determine the species thermochemistry. AURORA determines the bulk activities internally in a subroutine PSRACT. This subroutine can be easily modified by users who wish to specify their own relationships between the activities and the bulk-species mole fractions, temperature, and pressure. The default is to set the bulk activities, $a_{k}^{b}$, equal to their mole fraction in the bulk phase:

$$
a_{k}^{b}\left(T, P, X_{k}^{b}(n, m)\right)=X_{k}^{b}(n, m)
$$

More details regarding chemical reaction specifications, reaction-rate determinations, and thermochemical properties, are available in the CHEMKIN-III ${ }^{29,2}$ and SURFACE CHEMKIN-III user's manuals. $^{3}$

\section{Multiple Reactors in Series}

AURORA is capable of treating multiple well mixed reactors or PSRs that are connected in series. The procedure is to solve each PSR sequentially from 'upstream' to the most 'downstream' PSR in the series. The inlet flow rate and inlet composition of each downstream PSR is equal to the outlet flow rate and outlet composition of the adjacent upstream PSR. The user may specify different volumes, surface areas, surface temperatures, gas temperatures, heat-transfer rates, and total pressures for the different PSRs in the series. Additionally, different surfaces with different surface kinetics mechanisms may be 
included by using the materials specification in the SURFACE CHEMKIN input file and assigning different material surface-area fractions for each PSR.

\section{RF Sheath Model Option}

In plasma processing, it is often desirable to know the average ion energy of ions encountering a wafer that is being processed in a reactor. For many semiconductor processes, the wafer chuck is electrically biased through the application of an RF voltage or RF current. This RF bias will result in a time-averaged DC potential across the plasma sheath. Positive ions are accelerated by this potential as they traverse the sheath. Many analytical sheath models exist that, given the applied voltage or current amplitude and phase, determine the time-averaged sheath potential and energy gained by ions

encountering such a potential. AURORA includes one such model, based on the work of M. Riley, ${ }^{30}$ as an optional calculation available to the user. 


\section{NUMERICAL SOLUTION METHOD}

AURORA employs the numerical software package, TWOPNT ${ }^{22}$ in the solution of the algebraic equations described in the previous section. TWOPNT solves the system of algebraic equations by first applying a damped modified Newton algorithm to the set of $J$ nonlinear algebraic equations represented by the steady-state versions of Eqs. (3), (10), (19-21), (39), and (34). However, in the event that the Newton algorithm fails to converge during the iteration, the code automatically conditions the solution estimate by integrating the time-dependent version of the equations over a fixed number of time steps. This time-stepping procedure provides a new starting estimate for the Newton algorithm that is closer to the steady-state solution, increasing the likelihood of convergence for the Newton method. After time-stepping, the Newton algorithm tries again for convergence and, if this fails, TWOPNT takes additional time steps on the transient solution to further improve the initial iterate. Ultimately, the Newton iteration converges on the steady-state solution.

\section{Starting Estimates}

The use of a Newton iteration algorithm for solution of the governing equations requires the user to provide initial estimates of the solution variables. It may sometimes be difficult to obtain good initial estimates of species composition, especially when one is not familiar with the chemistry system being studied. In such cases, it may be necessary to approach the problem from points that are more intuitive. For example, in a thermal problem, a good initial estimate of gas species is often the thermodynamic equilibrium composition at the initial temperature. For this purpose, we have provided an optional call to the Stanford equilibrium routine, STANJAN, ${ }^{31}$ which minimizes the Gibb's free energy of the system in order to determine the equilibrium gas mole fractions. This method of determining the initial estimate of the gas-phase composition is automatically initiated in AURORA when the user does not supply any initial estimates for the gas-phase species' mole fractions. If equilibrium composition does not provide immediate convergence for the reactor conditions of interest, the user may increase the residence time (thereby driving the conditions closer to equilibrium) from the target conditions until a solution is reached. This solution can then be used as the initial estimate for the desired residence time. Such gradual approach to a desired solution is facilitated by the continuation capability (keyword CNTN) built into AURORA, and generally requires very little computational time. For a plasma system, a similar approach could be used to obtain a solution where the initial guess is difficult to obtain. In this case, the user might assume an initial guess for the gasphase composition close to that of the reagent gases entering the reactor, and set the power deposition level very low. Then gradually increasing the reactor power using the continuation capability can lead to the desired solution. 
Initial estimates of surface site fractions are often more difficult, but also have less impact on the ability of the Newton algorithm to reach a solution. A general rule of thumb for all species types is to provide an estimate for every species in the system, even if these numbers are small. An initial guess of $1 \times 10^{-7}$, for example, is generally much better than allowing the program to set the initial fraction to zero. All initial estimates will be normalized within AURORA, such that the sums of gas mole fractions, surface site fractions, and bulk species fractions are all equal to one.

AURORA provides an option to the user to either solve the gas energy equation or to keep the temperature fixed at the initial estimate. There are some cases when the user may choose not to solve the gas energy equation. For example, the reactor temperature may have been accurately measured, while heat losses are difficult to estimate. In cases where the energy equation is solved, the user has an option to solve the system of equations in one or two steps. The default is to use two steps: first solve for the species composition at the fixed temperature estimate provided by the user, and then solve simultaneously the energy equation and the species composition using the first solution as the initial guess. This two-stage method provide more robust convergence for thermal systems, where the reaction rates' exponential dependence on the gas temperature is the primary source of equation stiffness and nonlinearities. For plasma systems, when one is solving for the electron energy equation, convergence is usually most expedient with the one-step option (keyword NOFT).

\section{Modified Damped Newton's Method}

Newton's method determines a sequence of iterations or approximate solutions that approach the true solution. For the sake of notation simplicity, we call these approximate solution vectors, $\phi$. When an arbitrary $\phi$ is substituted into the governing equations, the equations generally do not equal zero unless $\phi$ also represents the true solution; in general the equations equal a residual vector $F$. The goal then is to find $\phi$ such that

$$
F(\phi)=0
$$

For AURORA, the vector $\phi$ is:

$$
\begin{aligned}
\phi=\left\{T, T_{e}, Y_{1}, \ldots, Y_{K_{g}}, Z_{K_{s}^{f}(1)}, \ldots, Z_{K_{s}^{l}(1)}, X_{K_{b}^{f}(1)}^{b}, \ldots, X_{K_{b}^{l}(1)}^{b}, \rho_{N_{\rho}^{f}(1)}, \ldots, \rho_{N_{\rho}^{l}(1)},\right. \\
\left.Z_{K_{s}^{f}(M)}, \ldots, Z_{K_{s}^{l}(M)}, X_{K_{b}^{f}(M)}^{b}, \ldots, X_{K_{b}^{l}(M)}^{b}, \rho_{N_{\rho}^{f}(M)}, \ldots, \rho_{N_{\rho}^{l}(M)}\right\}^{\mathrm{T}}
\end{aligned}
$$

where $T$ and $T_{e}$ are the gas and electron temperatures, the $Y^{\prime}$ s are the gas species mass fractions, the $Z^{\prime} s$ are the surface site fractions within each surface phase, the $X$ 's are the mole fractions of species in the 
bulk phases, and the $\rho$ s are the surface site densities of those surface phases whose site densities are allowed to change. The total number of unknowns, $J$, is defined by Eq. (41). The corresponding vector $F$ is composed of the residuals of the gas energy equation, the electron energy equation, the species equations, and the continuity equation for the surface site densities.

Provided the initial estimate $\phi^{(0)}$ of the solution is sufficiently good, Newton's method produces a sequence of iterates $\left\{\phi^{(n)}\right\}$ that converges to the solution of the nonlinear equations $F(\phi)=0$. The purest form of the Newton algorithm,

$$
\phi^{(n+1)}=\phi^{(n)}-\left(\frac{\partial F}{\partial \phi}\right)_{\phi}^{(n)} F\left(\phi^{(n)}\right),
$$

is usually difficult to implement in practice. Evaluating the Jacobian matrices, $\partial F / \partial \phi$, is time consuming, and convergence typically requires a very good initial estimate $\phi^{(0)}$. TWOPNT employs the usual remedies for these problems. First, TWOPNT retains the Jacobian matrix through several iteration steps, rather than updating at every iteration as indicated in Eq. (44). Thus, the Jacobian used at the current iteration, $J^{(n)}$, may be based on a solution that is several iterations old. The user can specify in the AURORA input the maximum number of iterations TWOPNT performs before calculating a new Jacobian (keyword NJAC). Second, the advancement of the iterate $\phi^{(n)}$ to $\phi^{(n+1)}$ is damped by a factor $\lambda^{(n)}$. The modified Newton algorithm is then,

$$
\phi^{(n+1)}=\phi^{(n)}-\lambda^{(n)}\left(J^{(n)}\right)^{-1} F\left(\phi^{(n)}\right)
$$

where $0<\lambda^{(n)} \leq 1$, and

$$
J^{(n)}=\left(\frac{\partial F}{\partial \phi}\right)_{\phi^{(m)}} ; 1<m \leq n
$$

Internally, TWOPNT will generate a new Jacobian whenever convergence with the old Jacobian fails. While Eq. (45) correctly indicates the relation between the new and the old iterate, TWOPNT does not compute the inverse of the Jacobian matrix, but rather solves a system of linear equations, $J^{(n)} \Delta \phi^{(n)}=F\left(\phi^{(n)}\right)$, for the undamped vector, $\Delta \phi^{(n)}$.

The TWOPNT algorithm determines the damping parameter $\lambda^{(n)}$ and the need for a new Jacobian based on several criteria designed to keep the iteration stable and within solution bounds. To accept a new solution iterate $\phi^{(n+1)}$, TWOPNT requires that the undamped steps decrease in magnitude, ${ }^{32}$ i.e.,

$$
\left|\left(J^{(n)}\right)^{-1} F\left(\phi^{(n+1)}\right)\right|<\left|\left(J^{(n)}\right)^{-1} F\left(\phi^{(n)}\right)\right| .
$$


If the solution $\phi^{(n+1)}$ fails this criterion, TWOPNT rejects it and retries the step with half the damping parameter or a new Jacobian matrix. TWOPNT adjusts the damping parameter to ensure that the evolving solution always remains within the solution bounds specified by AURORA. Example of physical bounds that are imposed on the solution are: the temperature(s) must be positive, and the species mass fractions must be between zero and one. In AURORA it is actually possible to modify the lower bounds placed on the species mass fractions to be slightly negative (keyword SFLR). Allowing some species mole fractions to become slightly negative during iteration sometimes enhances the convergence rate, especially when the solution composition has species mass fractions that vary over many orders of magnitude.

The Newton iteration procedure continues along these lines until the user-defined convergence criteria are met. Convergence requires that the maximum norm of the undamped correction vector $\Delta \phi^{(n)}$ has been reduced to within user-defined absolute and relative tolerances, i.e.,

$$
|\Delta \phi| \leq \max (A, R \times|\phi|)
$$

where $A$ is the absolute tolerance (keyword ATOL) and $R$ is the relative tolerance (keyword RTOL). The relative tolerance roughly indicates the number of significant digits in the converged solution, and the absolute tolerance serves to exclude solution components smaller than $A$ from the relative convergence criterion. Typically, the absolute tolerance $A$ should be smaller than the maximum mass fraction of any species of interest. The user should be particularly careful in specifying $A$ for plasma solutions, since the small electron mass will result in a much smaller mass fraction for electrons than for other important species. The relative tolerance should be in the range of $10^{-3}$ to $10^{-4}$.

If damping does not produce a suitable correction vector, TWOPNT computes a new Jacobian. In the case when a new Jacobian has just been computed, and a damped Newton step still cannot produce a suitable correction vector, TWOPNT begins to take time steps. The time-stepping procedure is described in the section following the description of the Jacobian Matrix below.

\section{Jacobian Matrix}

The Jacobian Matrix is a dense $J \times J$ matrix. Rather than derive and evaluate analytic expressions for the Jacobian elements, we form the elements of the Jacobian numerically, through finitedifference perturbations. This approach is justified since the accuracy of analytic Jacobians is not required for the modified Newton method described above. This is demonstrated by the successful use of old (and therefore inaccurate) Jacobians. We evaluate the numerical Jacobian elements from a onesided finite-difference formula as follows: 


$$
J_{i, j} \approx \frac{F_{i}\left(\phi_{j}+\delta\right)-F_{i}\left(\phi_{j}\right)}{\delta}
$$

where

$$
\delta=r \times \phi_{j}+a
$$

We choose the relative and absolute perturbations, $r$ and $a$, to be the square root of the computer's unit roundoff.

\section{Time Stepping Procedure}

The steady-state solution that we seek could be obtained through pure time-stepping using the transient equations described in Section II. Such a procedure is very reliable but also very slow. However, because the time-stepping is robust, we employ this procedure to condition the initial iterate in cases when the Newton's method is not converging. When the modified Newton's method fails, TWOPNT solves the transient equations for a given number of time steps, and then returns to the Newton method using the result of the time-stepping as the new initial iterate. This trial solution will be closer to the steady-state solution, and thus it is more likely to be within Newton's convergence domain.

The transient equations are Eqs. (2), (10), (19/20), (21), (39), and (34). We solve this system of ordinary differential equations using the backward-Euler method. In this method, the time derivatives are approximated by first-order, backwards finite differences. For example, $d T / d t$ is approximated as

$$
\frac{d T}{d t} \approx \frac{T_{j}^{n+1}-T_{j}^{n}}{\Delta t}
$$

where here the subscript $n$ indicates the time step index and $\Delta t$ represents the size of the time step. All other terms in the governing equation are evaluated at time level $n$. The time-discretized problem is then another system of nonlinear algebraic equations for the dependent variable vector $\phi$ at time level $n+1$.

To solve the system of equations for each time step we use the same Newton method as we employ in the steady-state problem. However, the transient problem is much more likely to converge; it should always converge for a sufficiently small time step. The objective, then, is to choose a time step (keywords TIME, TIM2) that is sufficiently large to make progress toward the steady solution, yet not so large as to introduce convergence difficulties in the transient solution method. Typical time steps 
range from 1-10 microseconds, although in plasma systems it is often necessary to choose time steps much smaller than this (10-100 nanoseconds).

After solving the specified number of time steps, TWOPNT again attempts to solve the steady problem by Newton's method. If the steady solution fails again the program reverts to time stepping, beginning where it left off at the last time-stepping procedure. Clearly the better the user's initial estimate of the solution, the less likely the program will have to resort to time stepping. If the program fails to converge in time-stepping, then, the user may either choose smaller time steps, or try a new starting estimate.

The time-stepping solution procedure described here is not particularly sophisticated, since we are neither interested in the accuracy of the transient path nor in solving the transient problem all the way to steady state. A much more accurate transient solution could be obtained using software such as $\mathrm{LSODE}_{r}{ }^{33}$ which was designed for the solution of stiff ordinary differential equations. We chose instead to use a method that is relatively inexpensive per step and employs the same Newton algorithm that is used in the steady-state solution. The transient solution therefore serves only to condition the starting estimates for the Newton's method, but does not provide accurate solution of the transient process.

\section{Sensitivity Analysis}

Sensitivity analysis of a problem solution allows quantitative understanding of how the solution depends on the various parameters contained in the model. The numerical method described above facilitates sensitivity analysis by having already computed the solution Jacobians. Once the Jacobian has been computed for the purposes of solving the nonlinear equations, the sensitivity coefficients are easily calculated. These techniques have been developed over many years and reported in the chemical engineering literature. ${ }^{34-36}$ We have found that sensitivity analysis is often an invaluable tool in interpreting the results of stirred reactor ${ }^{11}$ and flame experiments. ${ }^{37}$

In AURORA, we consider the first-order sensitivity coefficients of the gas temperature, species fractions, and bulk-phase growth rates with respect to the reaction-rate coefficients (keywords SENT, SENS, SENG). To specify the sensitivity coefficients, we begin by rewriting Eq. (42), which represents the vector of governing equations, as

$$
F(\phi(\alpha) ; \alpha)=0
$$

Here, we have introduced the idea that the equation may depend not only on the solution variables but also on a set of model parameters, $\alpha$. The residual vector $F$ depends on $\alpha$ both explicitly and implicitly 
through the solution vector $\phi$. In AURORA we consider the $\alpha$ 's to represent the pre-exponential "Afactors" in the Arrhenius reaction-rate expressions. By differentiating Eq. (52) with respect to $\alpha$ we obtain a matrix equation for the sensitivity coefficients:

$$
\left.\frac{\partial F}{\partial \phi} \frac{\partial \phi}{\partial \alpha}\right|_{F}+\frac{\partial F}{\partial \alpha}=0
$$

The matrix $\partial F / \partial \phi$ is the Jacobian of the original system and $\partial F / \partial \alpha$ is the matrix of partial derivatives of $F$ with respect to the parameters. The sensitivity coefficients are defined as $\partial \phi / \partial \alpha$. It is helpful to think of the $\partial F / \partial \alpha$ matrix column by column, with each column indicating the dependence of the residual vector $F$ on each parameter. There are as many columns as there are parameters, i.e. the number of gas-phase and surface reactions. The sensitivity coefficient matrix $\partial \phi / \partial \alpha$ contains quantitative information on how each reaction-rate coefficient affects the temperature(s) and species fractions. The sensitivity matrix has a structure similar to that of the $\partial F / \partial \alpha$ matrix. That is, each column contains the dependence of the solution vector on a particular chemical reaction.

The Jacobian and its LU factorization are already available from the solution of the original system by Newton's method. Parameter derivatives are computed by finite differences in a manner similar to the computation of the Jacobian. We can therefore readily solve the linear system of equations represented by Eq. (53) for each column of the sensitivity matrix corresponding to the sensitivities of the solution vector to each of the reaction rates in the CHEMKIN and SURFACE CHEMKIN mechanisms. TWOPNT employs the LINPACK ${ }^{38}$ software to perform these computations. We factor the Jacobian only once, and compute each column of the sensitivity matrix by back substitution, such that the calculation is relatively inexpensive computationally.

AURORA further manipulates the raw sensitivity coefficients to make them more useful. First, we compute normalized sensitivity coefficients in the form of logarithmic derivatives, i.e.,

$$
\left.\frac{\partial \ln Y_{k}}{\partial \ln \alpha_{i}}\right|_{F}=\left.\frac{\alpha_{i}}{Y_{k}} \frac{\partial Y_{k}}{\partial \alpha_{i}}\right|_{F}
$$

or

$$
\left.\frac{\partial \ln T}{\partial \ln \alpha_{i}}\right|_{F}=\left.\frac{\alpha_{i}}{T} \frac{\partial T}{\partial \alpha_{i}}\right|_{F}
$$

Second, although the gas-phase species solution variables are mass fractions, the sensitivity coefficients are computed in terms of mole fractions, as follows:

$$
\left.\frac{\alpha_{i}}{X_{k}} \frac{\partial X_{k}}{\partial \alpha_{i}}\right|_{F}=\left.\frac{\alpha_{i}}{Y_{k}} \frac{\partial Y_{k}}{\partial \alpha_{i}}\right|_{F}-\left.\alpha_{i} \bar{W} \sum_{j=1}^{K_{g}} \frac{1}{W_{j}} \frac{\partial Y_{k}}{\partial \alpha_{i}}\right|_{F}
$$


where $X_{k}$ are the gas-phase mole fractions, $W_{j}$ are the species molecular weights, and $\bar{W}$ is the mean molecular weight of the gas-phase mixture.

AURORA also computes the normalized sensitivity coefficients for the surface-site fractions and bulk-phase mole fractions. They are defined as:

$$
\left.\frac{\partial \ln Z_{k}}{\partial \ln \alpha_{i}}\right|_{F}=\left.\frac{\alpha_{i}}{Z_{k}} \frac{\partial Z_{k}}{\partial \alpha_{i}}\right|_{F}
$$

and

$$
\left.\frac{\partial \ln X_{k}^{b}}{\partial \ln \alpha_{i}}\right|_{F}=\left.\frac{\alpha_{i}}{X_{k}^{b}} \frac{\partial X_{k}^{b}}{\partial \alpha_{i}}\right|_{F},
$$

where $Z_{k}$ is the surface-site fraction of surface species $k$, and $X_{k}^{b}$ is the mole fraction of the bulk species $k$.

In addition to the gas, surface, and bulk species sensitivity coefficients, we also compute the sensitivity coefficients for the growth (or etch) rates of the bulk phases with respect to the reaction rate coefficient parameters $\alpha_{i}$. First, the linear growth rate (units of $\mathrm{cm} / \mathrm{s}$ ) of the bulk phase $n$ can be expressed by:

$$
G_{n, m}=\sum_{k=K_{b}^{f}(n, m)}^{K_{b}^{l}(n, m)} \frac{W_{k}}{\rho_{k}} s_{k, m} .
$$

The derivative with respect to the parameters is then,

$$
\begin{aligned}
\left.\frac{d G_{n, m}}{d \alpha_{i}}\right|_{F} & =\left.\sum_{k=K_{b}^{f}(n, m)}^{K_{b}^{l}(n, m)} \frac{W_{k}}{\rho_{k}} \frac{d \dot{s}_{k, m}}{d \alpha_{i}}\right|_{F} \\
& =\sum_{k=K_{b}^{f}(n, m)}^{K_{b}^{l}(n, m)} \frac{W_{k}}{\rho_{k}}\left[\left.\left.\sum_{l=1}^{J} \frac{\partial \dot{s}_{k, m}}{\partial \phi_{l}}\right|_{\alpha_{i}, \phi_{p \neq l}} \frac{\partial \phi_{l}}{\partial \alpha_{i}}\right|_{F}+\left.\frac{\partial \dot{s}_{k, m}}{\partial \alpha_{i}}\right|_{\phi_{p}}\right] .
\end{aligned}
$$

The vector, $\left.\left(\partial \phi_{l} / \partial \alpha_{i}\right)\right|_{F}$ is the vector of raw sensitivity coefficients computed by solution of Eq. (53). $\left.\left(\partial \dot{s}_{k} / \partial \phi_{l}\right)\right|_{\alpha_{i}, \phi_{p \neq l}}$ is a matrix representing the derivative of the bulk species production rates (from surface reaction with respect to the solution variables. $W_{k}$ and $\rho_{k}$ are the molecular weight and mass density of the $k$ th bulk-phase species, respectively. As with the species sensitivity coefficients, AURORA reports the normalized growth rate sensitivity coefficients:

$$
\left.\frac{\partial \ln G_{n, m}}{\partial \ln \alpha_{i}}\right|_{F}=\left.\frac{\alpha_{i}}{\left|G_{n, m}\right|} \frac{\partial G_{n, m}}{\partial \alpha_{i}}\right|_{F}
$$


Note that, in general, $G_{n, m}$ can be either positive (growth) or negative (etch). Therefore, the absolute value of $G_{n, m}$ on the right-hand-side of Eq. (62) is necessary to correctly normalize the sensitivity coefficients.

\section{Rate of Production Analysis}

Another useful tool in understanding well mixed reactor calculations is rate-of-production analysis. Rate-of-production analysis determines the contribution of each reaction to the net production or destruction rate of a species. The molar production of a species per unit volume, $P_{k}$, is given by

$$
P_{k}=\dot{\omega}_{k}+\sum_{m=1}^{M} \frac{A_{m}}{V} \dot{s}_{k, m}=\sum_{i=1}^{I} v_{k i} q_{i}+\sum_{m=1}^{M} \frac{A_{m}}{V} \sum_{i=1}^{l_{s}^{s}} v_{k i}^{s} q_{i}^{s}
$$

where $v_{k i}$ and $v_{k i}^{s}$ are the stoichiometric coefficients for the gas and surface reactions, respectively, and $q_{i}$ and $q_{i}^{s}$ are the rate of progress of the $I$ gas-phase reactions and the $I^{s}$ surface reactions. The contribution to the rate of production of species $k$ from gas-phase reaction $i$ is therefore

$$
C_{k i}=v_{k i} q_{i}
$$

and for a surface reaction,

$$
C_{k i}^{s}=\frac{A_{m}}{V} v_{k i}^{s} q_{i}^{s}
$$

where $A_{m}$ is the surface area of the material $m$ with which the reaction $i$ is associated. Further details about how the reactions contribute to net species production rates are found in the CHEMKIN and SURFACE CHEMKIN user manuals. ${ }^{3,2}$ AURORA computes the normalized values of the reaction contributions to the species production and destruction rates (keyword ROP). The normalized production-contributions for gas-phase reactions are given by:

$$
\bar{C}_{k i}^{p}=\frac{\max \left(v_{k i}, 0\right) q_{i}}{\sum_{i=1}^{I} \max \left(v_{k i}, 0\right) q_{i}+\sum_{m=1}^{M} \frac{A_{m}}{V} \sum_{i=1}^{l^{s}} \max \left(v_{k i}^{s}, 0\right) q_{i}^{s}}
$$

and the normalized destruction values for the gas-phase reactions is given by:

$$
\bar{C}_{k i}^{d}=\frac{\min \left(v_{k i}, 0\right) q_{i}}{\sum_{i=1}^{I} \min \left(v_{k i}, 0\right) q_{i}+\sum_{m=1}^{M} \frac{A_{m}}{V} \sum_{i=1}^{I^{s}} \min \left(v_{k i}^{s}, 0\right) q_{i}^{s}}
$$

The normalized production values for the surface reactions are given by: 


$$
\bar{C}_{k i}^{p}=\frac{\frac{A_{m}}{V} \max \left(v_{k i}^{s}, 0\right) q_{i}^{s}}{\sum_{i=1}^{I} \max \left(v_{k i}, 0\right) q_{i}+\sum_{m=1}^{M} \frac{A_{m}}{V} \sum_{i=1}^{I^{s}} \max \left(v_{k i}^{s}, 0\right) q_{i}^{s}}
$$

and the normalized destruction values for surface reactions are given by:

$$
\bar{C}_{k i^{s}}^{d}=\frac{\frac{A_{m}}{V} \min \left(v_{k i}^{s}, 0\right) q_{i}^{s}}{\sum_{i=1}^{I} \min \left(v_{k i}, 0\right) q_{i}+\sum_{m=1}^{M} \frac{A_{m}}{V} \sum_{i=1}^{I^{s}} \min \left(v_{k i}^{s}, 0\right) q_{i}^{s}}
$$

Thus, the normalized contributions to production and destruction sum to one, as follows:

and

$$
\sum_{i=1}^{I} \bar{C}_{k i}^{p}+\sum_{i=1}^{I^{s}} \bar{C}_{k i^{s}}^{p}=1,
$$

$$
\sum_{i=1}^{I} \bar{C}_{k i}^{d}+\sum_{i=1}^{I^{s}} \bar{C}_{k i^{s}}^{d}=1 .
$$




\section{PROGRAM STRUCTURE}

The AURORA code has a modular, top-down memory-management structure. The program calls subroutines from several software packages that have been in existence for some time and are in use for a variety of applications. These software packages include CHEMKIN, for gas-phase species and reaction information; SURFACE CHEMKIN, for surface species and reaction information; TWOPNT, the modifiedNewton-iteration solver described in Section III; and LINPACK, a set of linear algebra routines used in the Jacobian factorization and sensitivity coefficient computations. The AURORA code itself is written as a subroutine that may be called from a user-supplied driver routine. We provide an example of such a driver routine both within this manual and as part of the AURORA software as distributed. The driver routine performs the function of allocating total memory usage through definition of array sizes, as well as opening input and output files. AURORA checks internally to make sure that the allocated work arrays are sufficiently large to address the problem described by the CHEMKIN and SURFACE CHEMKIN input files. The driver routine is therefore the code most likely to be altered by the user as he or she moves from one chemistry application to another.

The use of CHEMKIN and SURFACE CHEMKIN subroutines requires that the user first execute the CHEMKIN and SURFACE CHEMKIN Interpreters, providing the necessary input for these routines. Figure 2 shows the relationship between AURORA, CHEMKIN, and SURFACE CHEMKIN routines. The first step in setting up a problem in AURORA is to execute the CHEMKIN interpreter. The CHEMKIN Interpreter looks for an input file called 'chem.inp,' which contains the user-supplied information on the species, chemical reactions, and thermodynamic data for the gas-phase chemistry mechanism. If the thermodynamic data is not supplied in the 'chem.inp' file, the Interpreter will look for the thermodynamic database file 'therm.dat.' The CHEMKIN interpreter creates a binary file called 'chem.bin' containing all of the gas-phase chemistry information, as well as an ASCII output file 'chem.out' describing the user input. The 'chem.bin' file is used both by the SURFACE CHEMKIN Interpreter, and by the AURORA program to allow access of the gas-phase chemistry information by the routines contained in the CHEMKIN Gas-phase Subroutines Library. Once the CHEMKIN Interpreter has been executed, the user must execute the SURFACE CHEMKIN Interpreter. This is necessary even in the case where no surface chemistry is included in the problem of interest. The SURFACE CHEMKIN Interpreter looks for a file called 'surf.inp,' which contains surface and bulk species information and surface reaction descriptions, as well as thermodynamic data for the surface and bulk species. Again, if the thermodynamic data is not supplied in the 'surf.inp' file, the Interpreter looks for the file 'therm.dat.' In the case when no surface reactions are included, the 'surf.inp' file would only contain an 'END' statement. Similarly to the CHEMKIN Interpreter, the SURFACE CHEMKIN Interpreter creates a binary 'surf.bin' file and an ASCII 'surf.out' file. The 'surf.bin' file is read into AURORA so that the information is available to the routines contained in the SURFACE CHEMKIN Subroutine Library. 
Detailed examples of CHEMKIN and SURFACE CHEMKIN input files are included in the example problems described in Sections VI and VII.

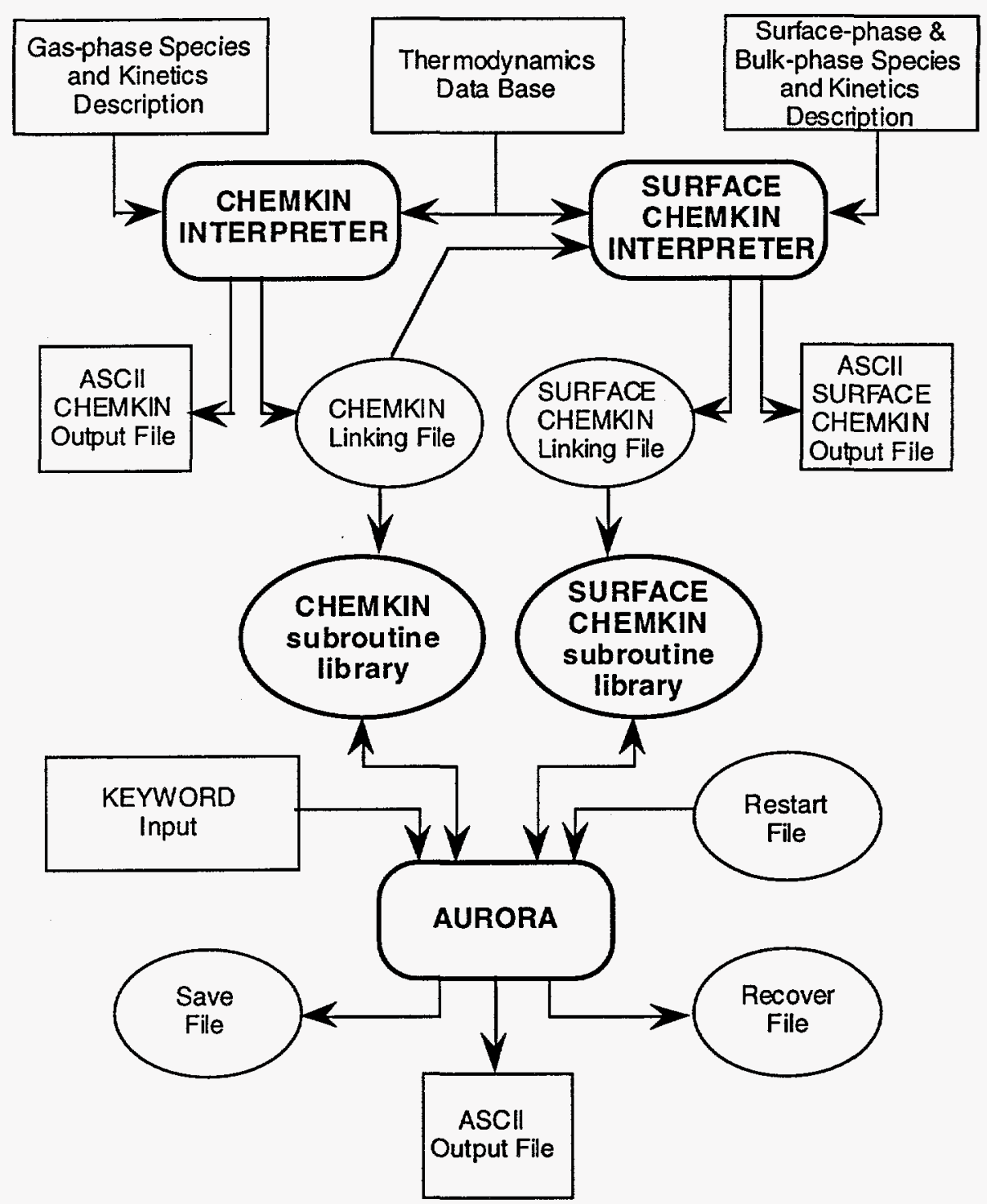

Figure 2. Relationship between AURORA, CHEMKIN and SURFACE CHEMKIN Interpreters, and Associated Input and Output files 
After executing the CHEMKIN and SURFACE CHEMKIN Interpreters, the user need only define the well mixed reactor parameters, which are contained in the keyword input file. The driver routine for AURORA typically reads this keyword input from Standard Input (FORTRAN Unit 5) and creates an ASCII output file on Standard Output (FORTRAN Unit 6), allowing command-line assignments of input and output files on UNIX systems. In addition to the ASCII output file, AURORA creates a binary output Save file, 'save.dat,' and intermediate solution Recovery files 'recov.dat.' The binary files are much more compact than the ASCII files and contain one or more complete solutions. More than one solution may be created in the 'save.dat' file using the continuation keyword (CNTN). These solutions are included sequentially, using identical format for each solution, in the Save file. AURORA may be started from 'scratch' where the user supplies the initial solution estimate in the input keyword file, or it may be started using a Restart file, 'restart.dat.' The Restart, Recovery, and Save files all have exactly the same format. In this way the user may copy 'save.dat' to 'restart.dat' to obtain the Restart file for a new set of conditions. AURORA will use the first solution found on the Restart file when a restart is desired.

The Save file is useful in post-processing the AURORA solution data. For example, when several continuation runs have been made, each using a different reactor residence time, the user may want to plot solution variables as a function of the residence time. The Save file may be probed by a post-processor routine to obtain this information. While such graphics routines are not provided with AURORA, we do describe the Save file format in this section in detail to allow the user to easily read this file into their own graphics program.

\section{Structure of the Save, Recover, and Restart Files}

The save file is a binary file that is written with the lines of FORTRAN shown in Figure 3. There are two lines of header and version information, followed by four sets of records: CHEMKIN and SURFACE CHEMKIN pointers and storage arrays; solution variables; sensitivity coefficients; and rateof-production reaction contributions. Each set of records begins with a CHARACTER 18 variable that identifies the records that follow as either 'CHEMISTRY,' 'SOLUTION,' 'SENSITIVITY,' or 'RATE OF PRODUCTION,' data. The header and chemistry records are only written once to Save and Recover files. Only the latest solution will be written to the Recover file, while more than one solution may be sequentially written to the Save file (with the CNTN keyword or with the NPSR keyword set $>1$ ).

The Chemistry data includes one record that contains all of the CHEMKIN pointers used internally in the CHEMKIN Gas-phase Subroutine Library. It is not necessary for the user to know what is contained in this list. Instead, the user can read this information in from the binary file using the CHEMKIN subroutine, 'CKPNT.' An example of how this is done is shown in Figure 4. Similarly for SURFACE CHEMKIN, a list of pointers is written for each surface material contained in the surface 
The CHEMKIN storage array are written after the list of pointers. The sizes of the integer, real, and character arrays are LENI, LENR, and LENC, respectively. These values are included in the pointer list and are returned in the CKPNT subroutine call (See Fig. 4). For the SURFACE CHEMKIN storage arrays, there may be more than one material for which a reaction mechanism was defined in the SURFACE CHEMKIN input file. The total number of materials included in the surface mechanism is NMAT. Within AURORA, the storage arrays for each material are stored sequentially in single integer, real, and work arrays. When reading this information back, it is therefore necessary to calculate the starting place for each material. In Fig. 3, the starting places for each material in the integer, real, and character arrays are IMI(IM), IMR(IM), and IMC(IM), where IM is the index of the material. These pointers can be easily calculated from the information returned by SKPNT, as shown in Fig. 4.

The writing of solution variables begins with index or problem size information. NATJ is the total number of solution variables in the problem (see Eq. (41)), IPSR is the index for the current PSR (may be greater than one when calculating multiple PSRs in series), NPSR is the total number of PSRs in series, NMAT is the number of materials in the surface mechanism, and NSPHCH is the total number of surface phases whose surface site densities must be considered as solution variables for each material. NSPHCH is greater than zero if the surface kinetics mechanism contains reactions that do not conserve surface site densities. The index information is followed by reactor parameters. EQUIV is the equivalence ratio (for combustion problems), $P$ is the pressure [dynes $/ \mathrm{cm}^{2}$ ], TAU is the residence time [seconds], FLRT is the mass flow rate $[\mathrm{g} / \mathrm{sec}], \mathrm{V}$ is the reactor volume $\left[\mathrm{cm}^{3}\right]$, AREA is the total surface area in the reactor $\left[\mathrm{cm}^{2}\right], Q$ is the heat loss [cal/sec], TSURF is the temperature of the surfaces of the reactor (which may be different from the gas temperature), HTRN and TAMBIENT are the heattransfer coefficient and ambient temperature governing heat losses to the reactor surroundings, LHTRN is a LOGICAL flag indicating whether a heat-transfer coefficient or heat loss was specified, and AFRAC is the fraction of the total area corresponding to each surface material. TIN and XIN(K) are the inlet temperature $[\mathrm{K}]$ and inlet gas mole fractions, and KKGAS is the number of gas-phase species. The index (IPSR) on reactor parameters indicate that these parameters may differ from one PSR to another in series PSRs. KK is the total number of species. Note that $(\mathrm{NATJ}=2+\mathrm{KK}+\mathrm{NSPHCH})$.

In the next record, KEL is the index of the electron in the array of gas-phase species. The value of KEL can be obtained by calling the CHEMKIN III subroutine, PKINDX, as shown in Fig. 4. If KEL is zero, there are no electrons in the chemistry mechanism and the next record is not written to the Save or Recover files. If KEL is not zero, then we assume that the reactor is a plasma reactor and additional parameters describing the plasma conditions are written to the Save and Recover files. TEIN is the electron temperature at the inlet (usually set to be equal to TIN), POWR is the total source power deposited into the plasma [erg/s], TIONP is the ion temperature [K], BHMXI is the transportlimitation factor for the Bohm criterion, EIONSH and ESHTH both represent the energy that ions gain traversing the sheath at each material, using two different calculation techniques. These options are 
described in more detail in Section V. QLEX is an inelastic electron-collision loss rate that allows inclusion of losses supplemental to those specified directly in the CHEMKIN mechanism.

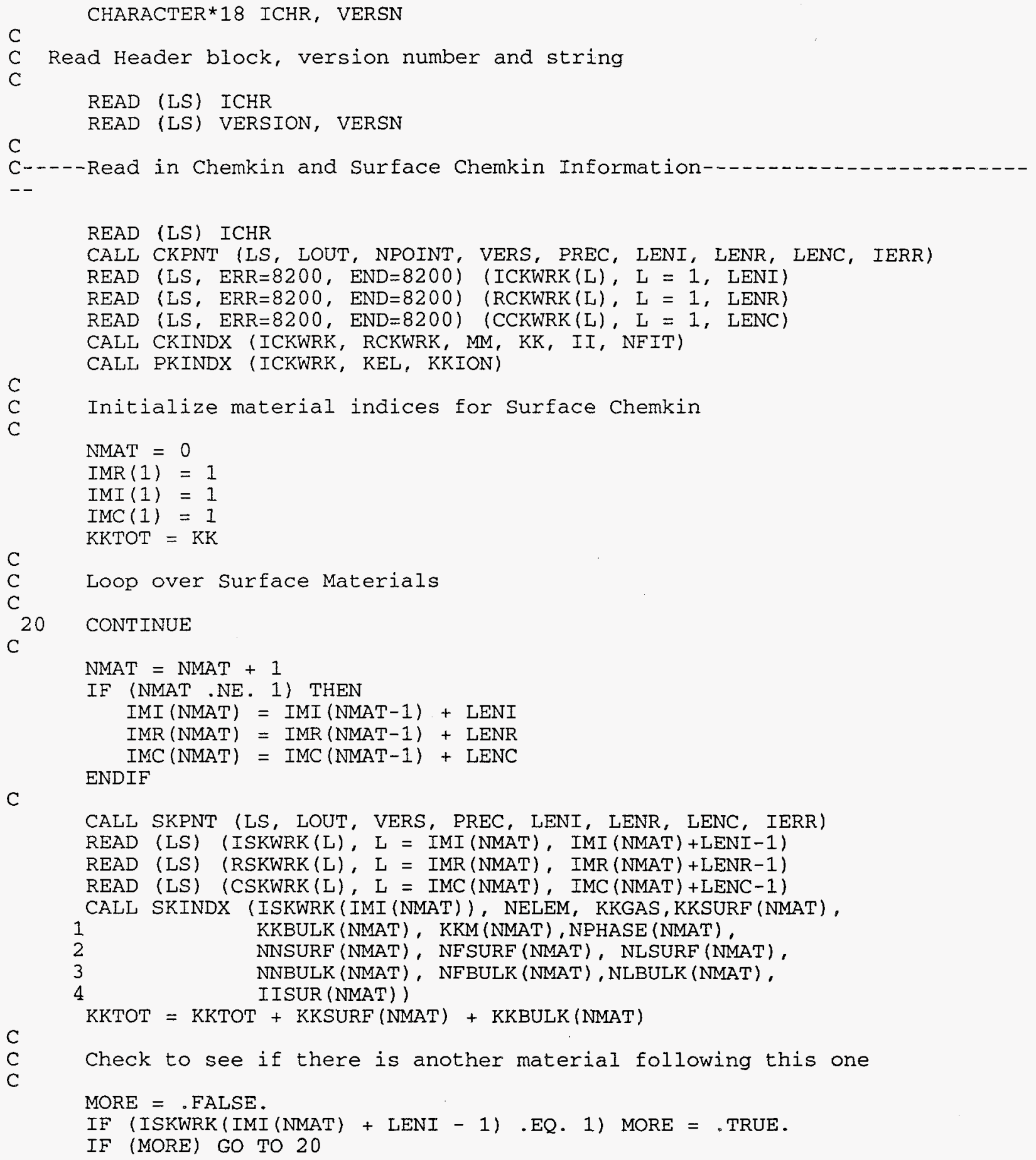

Figure 4(a). Lines of FORTRAN used to read the Save, Recover and Restart Files. (a) Reading in Chemistry Data 


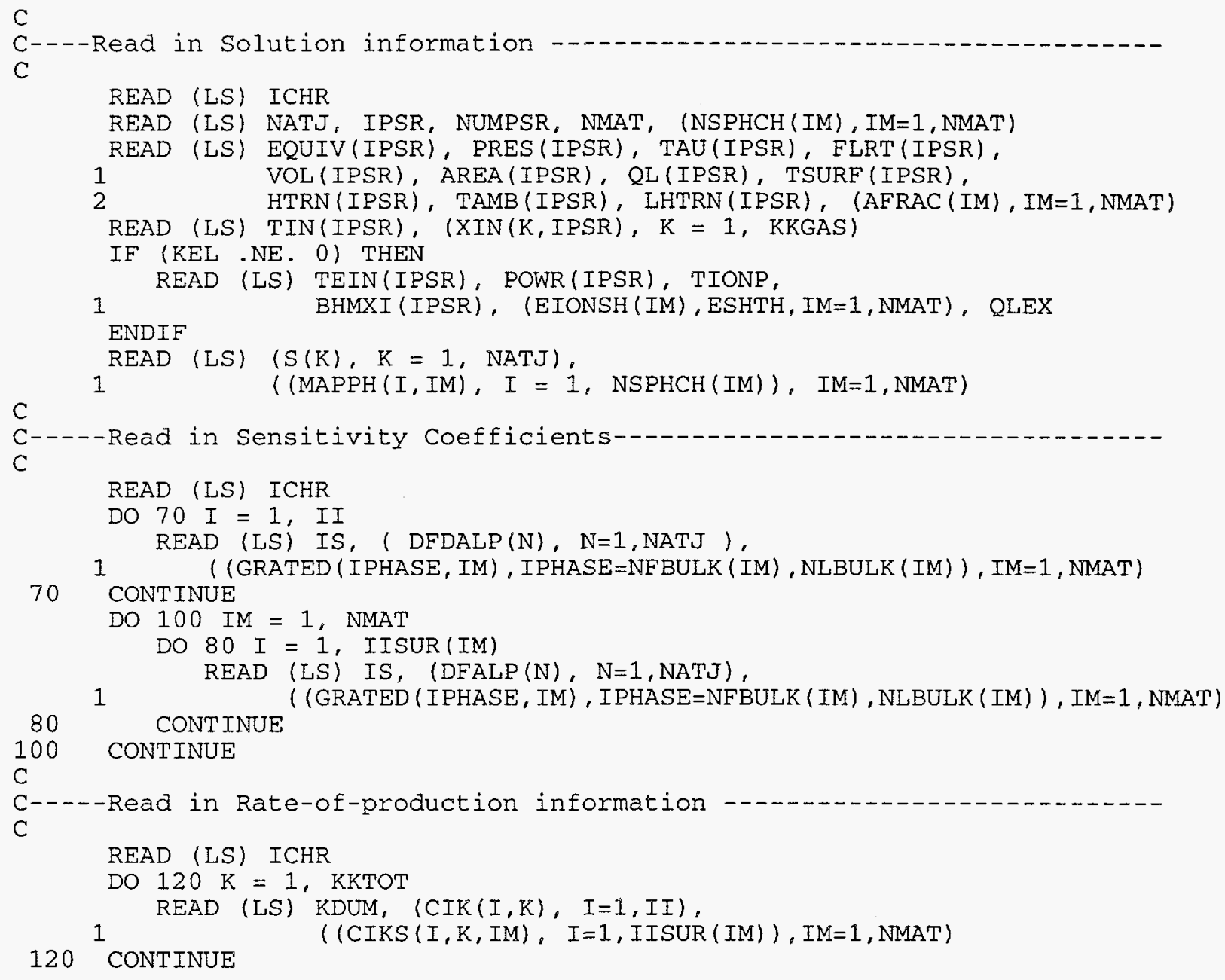

Following the plasma parameters in Fig. 3 , the solution variables are written. The solution vector $\mathrm{S}$ contains the variables $T, T_{e}$, and $Y_{k}$ for all gas-phase species. For each material, the solution vector contains $Z_{k}$ for all surface-phase species, and $X_{k}$ for all bulk-phase species. The species are ordered as they were read in from the CHEMKIN and SURFACE CHEMKIN input files. In the solution record, MAPPH is $\rho_{n}$ for all surface site densities that are allowed to change.

The sensitivity coefficients follow the solution record, when they are calculated. The calculation of sensitivity coefficients is a user option (keywords SENT, SENS, and SENG). DFDALP represents the first-order sensitivity coefficients $\partial \phi / \partial \alpha$ for each solution variable. Note that in Fig. 3 we have not shown the calculation of these coefficients for each reaction number, so that it seems like the same vector is being written repeatedly. In fact these calculations are within the I and IISUR 
loops, such that each vector represents a column in the $\partial \phi / \partial \alpha$ matrix. Likewise, in Fig. 4, we have not shown the mapping of these vectors into a two-dimensional matrix, although the user would probably want to do this in any post-processing routine. Following the sensitivity coefficients is the record containing the rates of production of each species by each reaction. Again, we only indicate in Figs. 3 and 4 the order and number of these entries, but have not shown the calculation from or mapping to twodimensional arrays.

\section{The Driver Program for AURORA}

The AURORA is designed to be called as a subroutine. Here we provide a sample driver routine that allocates work space, opens input and output files, and calls the AURORA code. This routine can be easily modified to change the size of the problem that can be handled by the AURORA program. In addition the user may wish to replaced this routine by one that serves the same purpose, but may have a much more sophisticated user interface, for example, or may include machine-dependent postprocessing routines.

Figure 5 shows an example of the user routine that we typically use to run AURORA. The routine begins by setting the problem to be double precision or single precision. Similar 'Change Blocks' are included within the AURORA code, and TWOPNT code, as well as the CHEMKIN and SURFACE CHEMKIN interpreters and libraries. It is essential that all these FORTRAN routines are set consistently to be either double precision or single precision. In our distribution of CHEMKIN and SURFACE CHEMKIN, we provide a routine called CHANGE that will interactively convert the FORTRAN source code from double precision to single precision and vise versa, when such Change Blocks are present in the code. For more information on this utility, the user may consult the CHANGE manual. ${ }^{39}$ Following the Change Blocks in the AURORA driver routine, we introduce integer variables and set the program size through a parameter statement. LNIWRK, LNRWRK, LNCWRK, and LNLWRK are the lengths of arrays for integer, real (floating-point), character, and logical work space, respectively. LIN and LOUT are the input and output file units, which we set to standard input and output. LINKCK, LINKSK, LREAD, LSAVE, and LRECOVER are the FORTRAN unit numbers used for opening the CHEMKIN linking file, 'chem.bin,' the SURFACE CHEMKIN linking file, 'surf.bin,' the AURORA Restart File, the AURORA Save File, and the AURORA Recover File. Open statements follow that are compatible with VAX VMS, UNIX, and PCs. Since we have used standard input and output units, the method for assigning input and output files may vary from one machine to another. Finally, the routine calls the subroutine PSR which is the internal driver for the AURORA code. 


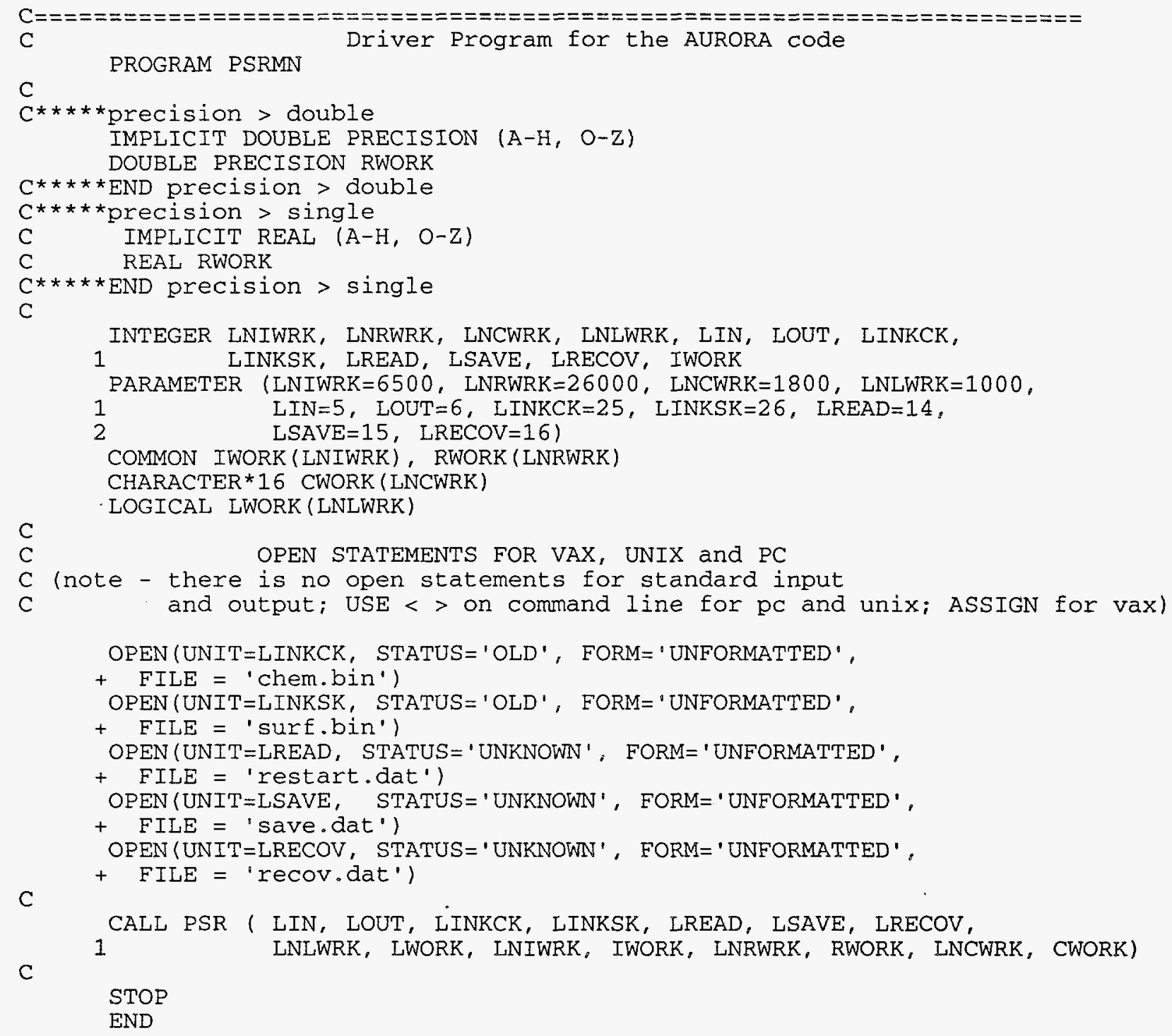

Figure 5. Example Driver Routine for AURORA 


\section{PROGRAM INPUT AND OUTPUT}

The user inputs information to AURORA using a keyword format. Each input line starts with an identifying keyword. For some keywords, only the keyword itself is required, while others require one or more pieces of additional information. Many keywords have default values associated with them. If these default values are appropriate for the user's problem, then these keywords do not need to be included in the input file. In the case of restarts or continuation problems, some of the parameters can be changed from what was used in the previous solution. If these keywords are not included or not changed in the input keyword list for continuations or restarts, then the parameters will retain their former values. In the list of keywords that follow, we indicate whether or not each keyword may be changed on a restart from a previous solution. The order of the keyword input is not important. The general rules governing the syntax of the keyword lines are listed below:

1. The first four characters of the line are reserved for the keyword, and it must begin at the first column.

2. Any further input associated with the keyword can appear anywhere in columns 5 through 80 . The specific starting column is unimportant.

3. When more than one piece of information is required, the order in which the information appears is important.

4. When numbers are required as input, they may be stated in either integer, floating point, or E format. AURORA converts the numbers to the proper type internally. The double precision specification $D$ is not recognized; however, the double precision conversion will be done internally, as necessary.

5. When species names are required as input, they must appear exactly as they are specified in the CHEMKIN and SURFACE CHEMKIN Interpreter input files. They must also appear in capital letters.

6. When more than one piece of information is required, the pieces are delimited by one or more blank spaces.

7. If contradictory or duplicate keywords are input, AURORA uses the information that is last read. Under some circumstances, this will result in a warning printed to the output file.

8. A "comment" line can be inserted by placing either an exclamation point (!), a period, (.), or a slash $(/)$ in the first column. Such a line is ignored by the code, but it is echoed back in the printed output. In addition, on any keyword line, any input that follows the required input and is enclosed in parentheses is taken to be a comment.

9. The keyword END must be the last input card. 


\title{
Keyword Descriptions
}

\author{
Problem Type
}

TGIV-Inclusion of this keyword means that AURORA will not solve the gas energy equation, but will instead use a fixed user-supplied temperature (see TEMP). An optional number on the keyword line specifies the PSR number. If no number is given, the keyword is assumed to apply to all PSRs in series.

Default-none; either TGIV or ENRG must be specified for each PSR

Restart-can be changed.

ENRG-Inclusion of this keyword means that AURORA will solve the gas-energy equation. An optional number on the keyword line specifies the PSR number. If no number is given, the keyword is assumed to apply to all PSRs in series. The user must still specify a temperature (see TEMP), which provides the initial guess for the gas temperature.

Default-none; either TGIV or ENRG must be specified for each PSR.

Restart-can be changed.

TEGV - This keyword will cause the electron temperature to be fixed at a user-specified value (see ETMP), rather than solving the electron energy equation. An optional number on the keyword line specifies the PSR number. If no number is given, the keyword is assumed to apply to all PSRs in series.

Default--none; either TEGV or ENGE must be specified when electrons are present.

Restart--can be changed.

ENGE-This keyword will cause the electron energy equation to be solved. An optional number on the keyword line specifies the PSR number. If no number is given, the keyword is assumed to apply to all PSRs in series. The user must still specify a temperature (see ETMP), which provides the initial guess for the electron temperature.

Default--none; either TEGV or ENGE must be specified when electrons are present.

Restart--can be changed.

NOFT-When this keyword is specified and an energy equation is being solved (ENRG or ENGE), the intermediate solution at a fixed temperature will be skipped. In this case, solution to the energy and species equations will be attempted simultaneously from the user-specified initial guess.

Default--AURORA will find the intermediate, fixed-temperature solution

Restart--can be changed. 
NPSR-Number of PSRs in series. Currently the maximum number of PSRs in series that the program can consider is 10 . This number can be changed, however, but modifying a parameter statement in AURORA.

Default-1

Restart-can be changed.

NSDN-Inclusion of this keyword indicates that under no circumstances should surface site densities be allowed to change, even if surface reactions that do not conserve surface sites are included in the SURFACE CHEMKIN mechanism. This option may be useful for aiding convergence in problems where the surface site densities may change, i.e., it may be helpful to first fix the surface-site densities to provide a better initial guess to the full problem.

Default-include surface site densities as solution variables when appropriate.

Restart-can be changed.

ETCH-Inclusion of this keyword indicates that a given bulk phase is expected to be etched instead of grown. The name of the bulk phase is required, except when there is only one bulk phase. Note that the default name of unnamed SURFACE CHEMKIN bulk phases is: BULK1, BULK2, etc. This option changes the form of the equations to be solved for the bulk phase composition, as described in Section II. When the keyword 'ETCH' is supplied for a bulk phase, it is required that 'BULK' keywords are also included for each bulk-phase species in that phase that is etched.

Default-assume all bulk phases are growing.

Restart-can be changed.

\section{Solution Method Options}

ATOL-Absolute tolerance for the termination of the Newton iteration (refer to SSABS in the TWOPNT manual ${ }^{22}$ ). The Newton iteration is considered to be converged when the maximum norm of the solution correction vector $\Delta \phi$ is reduced to less than the following criteria: $|\Delta \phi| \leq \max ($ ATOL, RTOL $\times|\phi|)$. Typically ATOL should be smaller than the maximum mass fraction of any species of interest.

Default-1.E-9

Restart-can be changed. 
RTOL-Relative tolerance for the termination of the Newton iteration (refer to SSREL in the TWOPNT manual ${ }^{22}$ ). The Newton iteration is considered to be converged when the maximum norm of the solution correction vector $\Delta \phi$ is reduced to less than the following criteria: $|\Delta \phi| \leq \max ($ ATOL, RTOL $\times|\phi|)$. Typically RTOL should be in the range of $10^{-3}$ to $10^{-6}$, which would provide roughly 3 to 6 digits of accuracy.

Default-1.E-4

Restart-can be changed.

ATIM-Absolute tolerance for the termination of the Newton iteration as it is used in the time stepping procedure (refer to TDABS in the TWOPNT manual ${ }^{22}$ ). For a precise definition, see the above description of ATOL. Since we are not seeking accuracy in a transient solution, this convergence criteria typically does not need to be as stringent as for the Newton iteration on the steadystate solution.

Default-1.E-9

Restart-can be changed.

RTIM-Relative tolerance for the termination of the Newton iteration as it is used in the time stepping procedure (refer to TDREL in the TWOPNT manual ${ }^{22}$ ). For a precise definition, see the above description of RTOL. Since we are not seeking accuracy in a transient solution, this convergence criteria typically does not need to be as stringent as for the Newton iteration on the steadystate solution.

Default-1.E-4

Restart-can be changed.

TIME-If the Newton method fails to converge, then the program takes some time steps in order to bring the current iterate within the domain of convergence of Newton's method (refer to STEPS1 in the TWOPNT manual ${ }^{22}$ ). This input specifies how many time steps to take and the initial size of the time step. For example, TIME 100 1.E6, specifies that the program will take 100 time steps, starting with a size of one microsecond.

Units-none; seconds

Default-100, 1.E6

Restart-can be changed

TIM2-This keyword is the same as TIME except that TIME applies to the fixed-temperature problem, while TIM2 applies to solutions where the energy equation is being solved. This input is only used when ENRG or ENGE is included.

Units-none; seconds

Default-100, 1.E6

Restart-can be changed 
DTMN-Minimum time step tolerated in TWOPNT's time stepping algorithm before flagging an error condition (refer to TMIN in the TWOPNT manual ${ }^{22}$ ).

Units-seconds

Default-1.E-10

Restart-can be changed.

DTMX-Maximum time step allowed in TWOPNT's time stepping algorithm (refer to TMAX in the TWOPNT manual ${ }^{22}$ ). When this value is reached, the time step size will no longer be increased and time stepping will continue with a fixed time step.

$$
\begin{aligned}
& \text { Units-seconds } \\
& \text { Default-1.E-4 } \\
& \text { Restart-can be changed. }
\end{aligned}
$$

IRET-Number of time steps to be taken in TWOPNT's time stepping algorithm before increasing the time step (refer to STEPS2 in the TWOPNT manual ${ }^{22}$ ).

$$
\text { Default-25 }
$$

Restart-can be changed.

UFAC-Factor by which to multiply the time step in TWOPNT's time stepping procedure when the number of time steps at the current step size reaches the number specified by 'IRET' (refer to TINC in the TWOPNT manual ${ }^{22}$ ).

Default -2.0

Restart-can be changed.

DFAC-Factor by which to divide the time step in TWOPNT's time stepping procedure when necessary, i.e., when the current time step does not converge (refer to TDEC in the TWOPNT manual ${ }^{22}$ ).

Default-2.0

Restart-can be changed.

NJAC-Number of iterations allowed in TWOPNT's Newton method before a new Jacobian is calculated (refer to SSAGE in the TWOPNT manual ${ }^{22}$ ).

Default-20

Restart-can be changed.

TJAC-Number of iterations allowed in TWOPNT's time-stepping procedure before a new Jacobian is calculated (refer to TDAGE in the TWOPNT manual ${ }^{22}$ ).

Default-20

Restart-can be changed. 
ISTP-Number of initial time steps to take before attempting Newton iterations for the steady-state problem (refer to STEPSO in the TWOPNT manual ${ }^{22}$ ).

Default-0

Restart-can be changed.

ABSL - This keyword is used to override the default value for the absolute perturbation in the solution variable used in the determination of the numerically derived Jacobian.

Units-none

Default-If the ATOL keyword is given, then the ABSL value is set equal to the ATOL value. If $A T O L$ is not specified, then the ABSL value is set equal to the square root of the unit roundoff error of the machine.

Restart-can be changed.

RELT - This keyword is used to override the default value for the relative perturbation in the solution variable used in the determination of the numerically derived Jacobian.

Units-none

Default-the square root of the unit roundoff error.

Restart-can be changed.

SFLR-This keyword is used to override the default value for the minimum bounds on the solution variables corresponding to gas-phase mole fractions, surface-phase site fractions, and bulkphase mole fractions. TWOPNT will not let the solution variables fall below their minimum bounds during iteration (refer to BELOW in the TWOPNT manual ${ }^{22}$ ).

Units-none

Default -0.0

Restart-can be changed.

\section{Reactor Conditions}

TEMP - The reactor gas temperature. Depending on the problem this is either the user supplied temperature (TGIV) or an initial estimate of the temperature (ENRG). An optional second number on the keyword line specifies the PSR number. If no number is given, the first value is assumed to apply to all PSRs in series.

Units-K

Default-required input for all PSRs.

Restart-can be changed. 
PRES-The reactor pressure in atmospheres. An optional second number on the keyword line specifies the PSR number. If no number is given, the first value is assumed to apply to all PSRs in series.

Units-atm

Default-required input for all PSRs.

Restart-can be changed.

PRMT - The reactor pressure in millitorr. An optional second number on the keyword line specifies the PSR number. If no number is given, the first value is assumed to apply to all PSRs in series.

Units-mtorr

Default-required input for all PSRs.

Restart-can be changed.

TAU-The nominal residence time of the gas in the reactor.

Units-sec

Default-none; either TAU, FLRT, or SCCM must be specified for the first PSR. For all subsequent PSRs in series, the flow rate of the preceding PSR is assumed to be the flow rate into the next PSR.

Restart-can be changed.

FLRT-The mass flow rate into the reactor.

Units- $\mathrm{g} / \mathrm{sec}$

Default-none; either TAU, FLRT, or SCCM must be specified for the first PSR. For all subsequent PSRs in series, the flow rate of the preceding PSR is assumed to be the flow rate into the next PSR.

Restart-can be changed.

SCCM-The volumetric flow rate into the reactor in standard cubic centimeters per second assuming that the inlet temperature is $298.15 \mathrm{~K}$ and the inlet pressure is $1 \mathrm{~atm}$.

Units- $\mathrm{cm}^{3} / \mathrm{sec}$

Default-none; either TAU, FLRT, or SCCM must be specified for the first PSR. For all subsequent PSRs in series, the flow rate of the preceding PSR is assumed to be the flow rate into the next PSR.

Restart-can be changed.

VOL-The volume of the reactor. An optional second number on the keyword line specifies the PSR number. If no number is given, the first value is assumed to apply to all PSRs in series.

Units- $\mathrm{cm}^{3}$

Default-none; required input for all PSRs.

Restart-can be changed. 
QLOS-The heat loss of the reactor. This keyword is only significant when the ENRG keyword is used. An optional second number on the keyword line specifies the PSR number. If no number is given, the first value is assumed to apply to all PSRs in series.

Units—cal/sec

Default-0.0; The QLOS and HTRN keywords are mutually exclusive for each PSR. If neither keyword is supplied for a PSR, then QLOS is assumed to have a value of 0.0 .

Restart-can be changed.

HTRN-The heat transfer coefficient and ambient temperature for specification of the heat loss from the reactor. This keyword is only significant when the ENRG keyword is used. An optional second number on the keyword line specifies the PSR number. If no number is given, the first value is assumed to apply to all PSRs in series.

Units- $h_{t}$ has units of cal $/\left(\mathrm{cm}^{2}-\mathrm{K}-\mathrm{sec}\right) ; T_{o}$ is in units of $\mathrm{K}$.

Default -0.0 ; The QLOS and HTRN keywords are mutually exclusive for each PSR. If neither keyword is supplied for a PSR, then QLOS is assumed to have a value of 0.0 .

Restart—can be changed.

AREA-The total surface area of in the reactor. An optional second number on the keyword line specifies the PSR number. If no number is given, the first value is assumed to apply to all PSRs in series.

Units- $\mathrm{cm}^{2}$

Default- 0.0

Restart-can be changed.

AFRA - Fraction of the total surface area that corresponds to a surface material (see the multiple surface material capability under SURFACE CHEMKIN). For example, 'AFRA WAFER 0.001' indicates that the material 'WAFER' comprises $0.1 \%$ of the total reactor surface area. The material name must correspond to a material name declared in the SURFACE CHEMKIN input file or an error will occur.

Units-none

Default-1.0 for all materials

Restart-can be changed.

TSRF-The temperature of the surfaces in the reactor. Use only if you want the surface temperature, which controls the surface chemistry rates, to be different than the gas temperature. An optional second number on the keyword line specifies the PSR number. If no number is given, the first value is assumed to apply to all PSRs in series. 
PWRW - Total power deposition to the plasma in units of Watts.

$$
\begin{aligned}
& \text { Units-Watts } \\
& \text { Default-0.0 } \\
& \text { Restart-can be changed. }
\end{aligned}
$$

PWRE - Total power deposition to the plasma in cgs units.

$$
\begin{aligned}
& \text { Units-ergs/sec } \\
& \text { Default-0.0 } \\
& \text { Restart-can be changed. }
\end{aligned}
$$

PWRC - Total power deposition to the plasma in calories $/ \mathrm{sec}$.

$$
\begin{aligned}
& \text { Units-calories/sec } \\
& \text { Default-0.0 } \\
& \text { Restart-can be changed. }
\end{aligned}
$$

ETMP - The electron temperature in the reactor. This value is either used as the user-specified fixed electron temperature (when TEGV is included) or as the initial estimate of the electron temperature for solution iteration (when ENGE is included).

\section{Units-K}

Default-the electron temperature is set equal to the gas temperature

Restart-can be changed.

TION - Specified temperature of ions. In this version of the software, there is no separate energy balance that accounts for ion energy gain above the gas temperature. The ions may, however, be much hotter than the neutral species, and this is accounted here as an additional energy loss from the deposited power required to heat the ions to the assumed temperature.

\section{Units $-\mathrm{K}$}

Default-the ions have the same temperature as the neutral gas

Restart-can be changed.

There are three ways in AURORA to specify the ion energy at surfaces in the reactor. This energy represents the energy gained by the ion through a plasma sheath at the plasma/surface interface. The value of the energy can have two roles in the solution. First, it represents a fraction of the total power deposited into the plasma; therefore the energy flux of the ions must be subtracted off the total power to get the power deposited to the electrons. Second, it is possible that some surface reaction rates depend on the ion energy. Some capabilities for describing such reactions have been implemented recently in SURFACE CHEMKIN. The first option for assigning the ion energy is a direct specification in units of electron Volts using the IONE keyword. A second option is to use the ELSH option to define the ion energy in terms of the electron temperature. This is often a good approximation of the sheath voltage, and therefore the ion energy. A third option is more time consuming but probably 
more accurate for surfaces where an electrical rf bias has been applied. This option is the solution of an analytical sheath model that determines the time-averaged ion energy of each ion crossing an $\mathrm{rf}$ sheath with specified current or voltage amplitude and frequency. This option is selected using the keyword RFSH, and includes several additional keywords to describe the rf bias. For current-control the user must specify RFFQ and RFIA, while voltage control requires specification of RFFQ, RFVA and RFVD. When looking at the sensitivity of the solution to these various options, it may be useful to separate out the use of the ion energy in the kinetics and the use in determining the fraction of the deposited power that goes to the ions in the sheath. For this reason, AURORA will allow specification of both ELSH and IONE or RFSH. In the case that both are specified, ELSH will be used for the power deposition calculations, and a warning will be printed in the ASCII output.

IONE - Specified energy loss to ions in the sheath for each ion lost at a specified material. The energy that the ions gain in the sheath is specified in electron Volts. For example, 'IONE MATERIAL1 $30^{\prime}$ would result in an ion energy gain of $30 \mathrm{eV}$ as it crossed the sheath near the material MATERIAL1. This energy gain for the ions results in a reduced effective power deposition to the electrons (unless ELSH is also specified), as described in Section II.

$$
\begin{aligned}
& \text { Units- }-\mathrm{V} \\
& \text { Default- } 0.0 \\
& \text { Restart—can be changed. }
\end{aligned}
$$

ELSH - Specified energy loss to ions in the sheath for each ion lost at a specified material. The energy that the ions gain in the sheath is typically assumed to be the sheath voltage, which can be described as a multiplier of $k T_{e}$. The value given hear is the value of the multiplier. For example, 'ELSH MATERIAL1 $5.0^{\prime}$ ' would result in an ion energy gain of $5 k T_{e}$ as it crossed the sheath near the material MATERIAL1. This energy gain for the ions results in a reduced effective power deposition to the electrons, as described in Section II.

$$
\begin{aligned}
& \text { Units—none } \\
& \text { Default-0.0 } \\
& \text { Restart—can be changed. }
\end{aligned}
$$

RFSH-Inclusion of this keyword flags AURORA to solve an $\mathrm{rf}$ analytic sheath model to get the timeaveraged ion energy crossing the sheath at a given material. An optional integer flag after the material name can be included to indicate that the $\mathrm{rf}$ bias will be current controlled (1) or voltage controlled (0). For example, 'RFSH MATERIAL1 1' would indicate a current-controlled rf sheath at MATERIAL1. The ion energy gain calculated from the sheath model results in a reduced effective power deposition to the electrons (unless ELSH is also specified), as described in Section II.

Default-No rf sheath simulation; If no integer is included, the rf sheath is assumed to be current-controlled.

Restart-can be changed. 
RFFQ-The rf Frequency for an rf sheath. This keyword is only relevant when RFSH is specified.

$$
\begin{aligned}
& \text { Units-Hertz } \\
& \text { Default-13.56E6 } \\
& \text { Restart-can be changed. }
\end{aligned}
$$

RFIA-The rf current amplitude for current-controlled rf bias. This keyword is only relevant when RFSH is specified with current control.

$$
\begin{aligned}
& \text { Units-Amps } \\
& \text { Default- } 0.0 \\
& \text { Restart-can be changed. }
\end{aligned}
$$

RFJA-Same definition as RFIA. RFIA and RFIA result in the same action in AURORA.

$$
\begin{aligned}
& \text { Units-Amps } \\
& \text { Default- } 0.0 \\
& \text { Restart-can be changed. }
\end{aligned}
$$

RFVA-The rf voltage amplitude for voltage-controlled $\mathrm{rf}$ bias. This keyword is only relevant when RFSH is specified with voltage control.

$$
\begin{aligned}
& \text { Units-Volts } \\
& \text { Default- } 0.0 \\
& \text { Restart-can be changed. }
\end{aligned}
$$

RFVD-The DC component of the voltage for a voltage-controlled $\mathrm{rf}$ bias. This keyword is only relevant when RFSH is specified with voltage control.

$$
\begin{aligned}
& \text { Units - Volts } \\
& \text { Default-0.0 } \\
& \text { Restart-can be changed. }
\end{aligned}
$$

QLSE - This is an additional energy loss term for the electrons that is introduced to account for inelastic collisional losses that are not already included explicitly in the CHEMKIN reaction mechanism. Energy loss values are specified here as a function of electron temperature and are given per ionization event to be consistent with the work of Lee, et al. ${ }^{17}$ For example, 'QLSE 34800. 100.' would represent an electron energy loss of $100 \mathrm{eV} /$ ionization event for an electron temperature of $34800 \mathrm{~K}(3.0 \mathrm{eV})$.

Units-energy loss: $\mathrm{eV} /$ ionization event; electron temperature: $\mathrm{K}$

Default-0.0

Restart-can be changed. 
XSEK - Momentum-transfer collision cross section between electrons and a specified species. For example, 'XSEK AR 1.0e-16' would indicate a momentum-transfer cross-section of $10^{-16} \mathrm{~cm}^{2}$ between electrons and argon atoms. For any species not specified by the XSEK keyword, a userspecified default value will be used.

Units- $\mathrm{cm}^{2}$

Default-the value specified by XSDF below.

Restart-can be changed.

XSDF - Default value for a momentum-transfer cross-section between electrons and each species. This value is used for all species not specified with the XSEK keyword.

Units $-\mathrm{cm}^{2}$

Default-none; the user must either specify XSDF or include XSEK for all species except electrons.

Restart-can be changed.

BOHM-Inclusion of this optional keyword results in the constraint of ion fluxes to surfaces based on a modified Bohm criterion. The value input with the BOHM keyword represents the ratio of the ion density at the sheath edge over the ion density in the plasma bulk. Since AURORA does not calculate this spatial dependence of the ion density, this correction to the Bohm flux is necessary to obtain the correct ion loss to the reactor walls and other surfaces. A typical value for BOHM is 0.4-0.6. When this keyword is included, AURORA will correct the ion surface loss rate determined by SURFACE CHEMKIN routines, such that the flux of each ion is equal to its Bohm velocity multiplied by this Bohm correction factor. Note: AURORA also corrects the Bohm velocity to include the effect of negative ions in the plasma.

Units-none.

Default-none. If this keyword is not included, the ion flux will be determined solely from the SURFACE CHEMKIN rate information.

Restart-can be changed.

CNFN - Inclusion of this keyword results in the confinement of negative ions; i.e. the outflow term in the species balance for negative ions is neglected. This option is included to allow consistency with the work of Lee, et al. ${ }^{17}$

Default-negative ions are allowed to flow out of the reactor.

Restart-can be changed.

GFAC - Multiplier for all gas-phase reaction rates. This keyword can be useful for determining effects of and errors in the gas-phase chemistry, without rerunning the CHEMKIN interpreter.

Units-none

Default-1.0

Restart-can be changed. 
SFAC - Multiplier for all surface reaction rates. This keyword can be useful for determining effects of and errors in the surface chemistry, without rerunning the SURFACE CHEMKIN interpreter.

$$
\begin{aligned}
& \text { Units-none } \\
& \text { Default-1.0 } \\
& \text { Restart-can be changed. }
\end{aligned}
$$

\section{Inlet Conditions}

TINL-The inlet temperature.

\section{Units-K}

Default-none; required input for all problems where ENRG is specified.

Restart-can be changed.

TEIN - Electron temperature in the inlet stream. For most cases, there are no free electrons in the inlet stream, in which case the electron inlet temperature is not used.

\section{Units-K}

Default-the electrons have the same temperature as the inlet gas.

Restart-can be changed.

The inlet composition can be supplied in one of two ways. The most common way is to define the inlet mole fractions for each reactant species (REAC). An alternative is available for combustion problems involving mixtures of fuels and oxidizers. For this case, the user may define the equivalence ration (EQUI), the fuel composition (FUEL), the oxidizer composition (OXID) and the product species (PROD). AURORA then determines the inlet composition from these parameters.

REAC-Mole fraction of the reagent gases entering the reactor. One of these REAC inputs must appear for each species in the inlet gas stream. For example, REAC C2H2 0.5 , would indicate that acetylene has a mole fraction of 0.5 in the inlet gas. The sum of all the reactant mole fractions should equal to one. However, if they do not, AURORA will proceed to normalize the mole fractions so that they do sum to one, and print a warning message in the output file.

Units-none

Default-none; at least one REAC keyword is required, unless the EQUI /FUEL /OXID /PROD option is used.

Restart-can be changed. However, the REAC keywords must be changed as a set, not individually

EQUI-The air/fuel equivalence ratio

Default-none; either REAC or EQUI/FUEL/OXID/PROD is required.

Restart-can be changed. 
FUEL-This keyword defines the fuel mole fraction composition. It must be followed by a species name and then the mole fraction. One of these FUEL inputs must appear for each fuel species. Any given species can participate simultaneously as a fuel, oxidizer, or product. The sum of all the fuel mole fractions should equal one. If it does not, a warning message will be printed and the mole fractions will be normalized so the sum does equal one. (Note: the mole fractions are of the fuel itself, not for the entire composition)

Units-none.

Default-none; either REAC or EQUI/FUEL/OXID/PROD is required.

Restart-can be changed.

OXID-This keyword defines the oxidizer mole fraction composition. It must be followed by a species name and then the mole fraction. One of these OXID inputs must appear for each oxidizer species. Any given species can participate simultaneously as a fuel, oxidizer, or product. The sum of all the oxidizer mole fractions should equal one. If it does not, a warning message will be printed and the mole fractions will be normalized so the sum does equal one. (Note: the mole fractions are of the oxidizer itself, not for the entire composition)

Units-none.

Default-none; either REAC or EQUI/FUEL/OXID/PROD is required.

Restart-can be changed.

PROD-One of these PROD inputs must appear for each product species when the EQUI /FUEL /OXID /PROD option is used. Any given species can participate simultaneously as a fuel, oxidizer, or product.

Units-none.

Default-none; either REAC or EQUI/FUEL/OXID/PROD is required.

Restart-can be changed.

ADD-Mole fractions of species added to the inlet composition may be included optionally with this keyword when the EQUI /FUEL /OXID / PROD option is used. These species do not enter into the equivalence ratio computations. One species is entered per line.

Units-none.

Default-none; either REAC or EQUI/FUEL/OXID/PROD is required.

Restart-can be changed. However, the ADD keywords must be changed as a set, not individually. 
Solution Estimate

XEST-Estimated gas-phase mole fractions to begin the iteration. For example, XEST H2O 0.5 assigns an initial mole fraction of 0.5 to water vapor in the reactor. The sum of all the estimated mole fractions should equal one. If they do not sum to one, AURORA will proceed to normalize them to one, and will print a warning message in the printed output.

Default -0.0

SURF-Estimated surface-site fraction of a surface species to begin the calculation. For example, SURF $\mathrm{Ga}$ (s) 0.001 assigns the initial surface-site fraction of 0.001 to the $\mathrm{Ga}(\mathrm{s})$ surface species. The sum of all estimated surface-site fractions for each surface phase $n$ should equal one. If they do not sum to one, AURORA will proceed to normalize them to one, and will print a warning message in the printed output.

Default-0.0

BULK-Estimated bulk-species mole fraction to begin the calculation. This is required input for bulk species in bulk phases that are being etched (see ETCH). For example, BULK Ga(d) 1.0 assigns the estimated mole fraction of 1.0 to the $\mathrm{Ga}(\mathrm{d})$ bulk-phase species. The sum of all estimated bulk-phase mole fractions for each bulk phase $n$ should equal one. If they do not sum to one, AURORA will proceed to normalize them to one, and will print a warning message in the printed output.

Default -0.0

SDEN-Estimated surface site density of a surface phase. For example, SDEN GAAS 1.0E-8 assigns the estimated surface site density of $1.0 \mathrm{E}-8 \mathrm{moles} / \mathrm{cm}^{2}$ to the surface phase named GAAS. If this surface site density is treated as an unknown in the problem, then this keyword acts to provide an initial estimate for the variable. If the surface site density is not a solution variable, then this keyword serves to override the default (standard state) surface site density that was input in the SURFACE CHEMKIN input file.

Units-mole $/ \mathrm{cm}^{2}$

Default-the surface site density is equal to that provided in the SURFACE CHEMKIN input file.

$\underline{\text { Sensitivity Analysis Options }}$

ASEN-Inclusion of this keyword causes the calculation of the first-order sensitivity coefficients with respect to the gas-phase and surface chemistry rate constants for all species and the gas temperature.

Default-no sensitivity coefficients are computed. 
SEN- Inclusion of this keyword causes the calculation of the first-order sensitivity coefficients with respect to the gas-phase and surface chemistry rate constants for the specified species. The keyword should be followed by a species name. Note that all the sensitivity coefficients will be calculated and written to the Save File, but only those requested will be included in the printed output (Unless the ASEN, SENT, or SENG keyword is used).

Default-no sensitivity coefficients are computed.

SENT-Inclusion of this keyword causes the calculation of the first-order sensitivity coefficients with respect to the gas-phase and surface chemistry rate constants for the gas temperature. Note that all the sensitivity coefficients will be calculated and written to the Save File, but only the gas temperature sensitivities will be included in the printed output (Unless the ASEN, SEN, or SENG keyword is used).

Default-no sensitivity coefficients are computed.

SENG-Inclusion of this keyword causes the calculation of the first-order sensitivity coefficients with respect to the gas-phase and surface chemistry rate constants for the growth rate of all bulk phases. Note that all the sensitivity coefficients will be calculated and written to the Save File, but only the growth-rate sensitivities will be included in the printed output (Unless the ASEN, SEN, or SENT keyword is used)。

Default-no sensitivity coefficients are computed.

EPSS-Threshold value for the first-order sensitivity coefficients for the species with respect to the rate constants. Coefficients below this value are not printed. However all sensitivity coefficients that are calculated will be written to the Save File.

Default-0.001

EPST-Threshold value for the first-order sensitivity coefficients for the gas temperature with respect to the rate constants. Coefficients below this value are not printed. However all sensitivity coefficients that are calculated will be written to the Save File.

Default-0.001

EPSG-Threshold value for the first-order sensitivity coefficients for the growth rates of all bulk phases with respect to the rate constants. Coefficients below this value are not printed. However all sensitivity coefficients that are calculated will be written to the Save File.

Default-0.001 
Rate-of-Production Analysis Options

AROP-Inclusion of this keyword causes the rate-of-production coefficients to be determined for all species.

Default-no rate-of-production coefficients are computed.

ROP-Inclusion of this keyword causes the rate-of-production coefficients to be printed for specified species. The keyword must be followed by one or more names of species. If any ROP keywords are used, all the rate-of-production coefficients will be computed and written to the Save File. However, only those specified by the ROP keyword will be printed in the ASCII output. More than one species may be entered per line. If the line is blank after the ROP keyword, then all rate-of-production contributions are computed and written to the Save File, but none are printed.

Default-no rate-of-production coefficients are computed.

EPSR-Threshold value for the rate-of-production coefficients. Coefficients below this value are not printed to the ASCII output. However, all the rate-of-production values that are calculated are written to the Save File.

Default-0.01

\section{Miscellaneous Controls}

PRNT-Printing control. "PRNT 0" provides printed output for only the solution (plus sensitivity coefficients and rates-of-production coefficients, when requested). "PRNT 1" provides an additional summary of the iteration path from the solver. "PRNT 2" includes printing at every stage of the Newton iteration and time stepping procedure. More printing is sometimes helpful when diagnosing problems and trying to adjust the input parameters to optimize convergence. However, since the increased printing requires more function evaluations to show how the solution is progressing, the computer time increases with increased printing.

Default-1

Restart-can be changed.

RSTR-Inclusion of this keyword causes AURORA to read a solution off the Restart File and begin iteration on the current reactor conditions from this solution.

Default-solution started from the input estimates and no Restart File is used. 
CNTN-Inclusion of this keyword causes AURORA to expect keywords for another problem to follow the END keyword. The following problem uses the solution of the previous problem as its initial guess. This capability is very similar to that provided by RSTR. However, in the case of CNTN, several related problems can be solved by one job submission, without having to manipulate the restart files. The solutions resulting from CNTN keywords are written sequentially to one Save File. Any keyword that can be changed for a RSTR problem can be changed on a continuation.

Default-no continuation is expected.

END-This keyword signifies the end of the input data for a given reactor description. It must appear after each set of data when continuation jobs are indicated using the CNTN keyword. 


\section{SAMPLE PROBLEM A: THERMAL CVD OF $\mathrm{Si}_{3} \mathrm{~N}_{4}$}

The example we consider is $\mathrm{Si}_{3} \mathrm{~N}_{4}$ deposition from a SiF $4 \mathrm{NH}_{3}$ mixture in a low pressure reactor. This mechanism should only be considered as illustrative and not as a source of kinetic data on the $\mathrm{Si}_{3} \mathrm{~N}_{4}$ system. The pressure (PRES), $2.368 \times 10^{-3} \mathrm{~atm}$, is low enough that the assumptions behind the

perfectly stirred tank reactor model are not too bad. The reactor has a volume (VOL) of $2,000 \mathrm{~cm}^{3}$, a surface area (AREA) of $950 \mathrm{~cm}^{2}$, and an inlet flow rate of 11,300 standard cubic centimeters per second (SCCM). The inlet composition is defined by the mole fraction keywords (REACT). The mole fractions of $\mathrm{SiF}_{4}$ is assumed to be 0.14286 . The remainder of the inlet gas is $\mathrm{NH}_{3}$. The temperature of the reactor is not calculated from an energy equation (TGIV). It is fixed 1,440 K (TEMP). The surface temperature is assumed to be equal to the gas phase temperature (i.e., no TSRF keyword is supplied). We do not supply a solution estimate for the species concentrations (XEST). Since this is not a restart problem (RSTR), the equilibrium composition is calculated at $1,440 \mathrm{~K}$ and used as a starting point for the iterations. However, we do supply a solution estimate for the surface site concentrations (SURF) and bulk phase mole fractions (BULK).

This example was run on a SGI Indigo $(150 \mathrm{MHz})$ work station using the UNIX operating system and the FORTRAN compiler. Thus, the CPU times reported in the output are for this computer.

The first file required by the code is the input file for the CHEMKIN Interpreter, which defines the $\mathrm{SiF}_{4}-\mathrm{NH}_{3}$ gas-phase reaction mechanism. The next file required by the code is the input file for the SURFACE CHEMKIN Interpreter, which defines the surface species in the $\mathrm{Si}_{3} \mathrm{~N}_{4}$ surface phase and the $\mathrm{Si}_{3} \mathrm{~N}_{4}$ solid, which is defined in terms of two bulk phases, $\mathrm{SI}(\mathrm{D})$ and $\mathrm{N}(\mathrm{D})$. The surface reactions are also defined, here. Though it is not immediately obvious, the nature of the surface mechanism guarantees a bulk-phase stoichiometry of 3:4. The next file shown is the output from the AURORA code. After giving statistics on the working space requirements, the code echoes the keyword inputs. Following the keywords, the AURORA prints information about the kinetics mechanism, such as the total number of species and reactions, and whether there are any surface reactions that do not conserve surface sites. Next comes a section about the inlet conditions: the total mass flow rate, volumetric flow rate, and individual species flow rates in various units. Information about parameters supplied to TWOPNT are supplied next. These include the lower and upper bound on solution variables (SFLR), the number of time steps to try if Newton's method fails and the initial time-step increment (TIME and TIM2), the absolute and relative bounds on the maximum norm of the solution updates for both the steady-state (RTOL and ATOL) and time-dependent problem (RTIM and ATIM), and the absolute and relative deltas to be used in the numerical differencing algorithm that forms the Jacobian (ABSL and RELT). 
The next block of output is written by the subroutine PSPRNT and contains the initial guess for the solution sent to TWOPNT. The number of times it is called in the program depends on the value of the keyword PRNT. It prints out the reactor conditions, inlet gas-phase mole fractions, current values for the solution variables and growth rates of bulk phases. It also prints out the current values of the steady-state residuals.

We have set the PRNT keyword to 2 so that detailed diagnostics for the progress of iteration are provided. The next block of output comes from NEWTON (which attempts to solve the problem by Newton's method). It indicates that after computing four Jacobians and three new solution estimates, the Newton iteration failed because one of the surface-site fractions became less than the lower bound. The column headings in the report from Newton are as follows. The "function" value is the logarithm of the maximum norm of the residuals, i.e., $F(\phi)$ of Eq. (42). The "step size" is the logarithm of the maximum norm of the undamped Newton step $J^{-1} F$ from Eq. (44) The "trial value" is the logarithm of the function $n$ evaluated at $\phi^{(n+1)}$. The "trial step" is the logarithm of the maximum norm of the undamped step for the "next" iteration (see Eq. (47)). The code damps the Newton step for $\phi^{(n+1)}$ until the trial step is less than the current "step size". The next column is the logarithm of the damping coefficient. The last column reports the logarithm of the LINPACK ${ }^{38}$ estimate of the Jacobian's condition number each time a new Jacobian is computed.

The next block of output is the report from TIMSTP. The first column is a counter for the time step number. The next column is the logarithm of the maximum norm of the steady state residual, i.e., $F(\phi)$. The following column is the logarithm of the maximum norm of the change in the solution $\phi$ that occurred by taking the time steps. The last three columns report statistics on the Newton iteration that is used to take each time step. These include the number of Newton steps and the number of Jacobians required for a given time step. Each time a new Jacobian is evaluated the condition number is reported. When there is no entry in the Jacobian columns then a Jacobian from a previous time step is being used.

In this example, the time stepping makes steady (but not monotonic) progress in reducing the residual norms. Many steps are computed with little work. Choosing a larger time step may have been advisable on this problem. The residual norms ultimately have to decrease. With the PRNT option at 2 , the current solution is printed every time NEWTON or TIMSTP completes its task successfully. So in this case, the first NEWTON fails and the TIMSTP is called. After TIMSTP completes the required 50 steps, the current iteration is printed. This is followed by a report from NEWTON, indicating that now it succeeds in 43 steps with four Jacobians. The final solution from PSPRNT is printed, together with timing statistics for the computation.

At the end of the run, details concerning the outlet composition and flow rate are printed. A detailed species balance is also printed. In that section inlet flow rates, outlet flow rates, gas-phase production rates, gas-phase destruction rates, net surface-phase production rates are quoted for each gas-phase and surface-phase species. The last column, entitled "TOTAL_NET," quotes the net rate of 
production for each gas-phase, surface-phase, and bulk-phase species in the problem. If the AURORA equations were solved exactly, this column should have zero entries for all gas-phase and surfacephase species. Bulk-phase species do not necessarily have a zero net production rate, due to the fact that bulk phases may be growing or etching. A detailed element balance is supplied after the detailed species balance, where the element fluxes for the inlet, outlet, and bulk phases are computed.

Lastly, results from requested normalized sensitivity coefficient calculations and rates-ofproduction analyses are printed. These are the normalized sensitivity coefficients as defined by Eqs. (54) and (62). In the current problem, sensitivity coefficients for gas-phase species, $\mathrm{HF}$, $\mathrm{SiF}_{4}$, and $\mathrm{NH}_{3}$ and for the overall growth rates of all bulk phases were requested. The results show that both the composition of all three of these gas-phase species and the total growth rate is determined by surface reaction number 2 , the initial dissociative adsorption reaction involving $\mathrm{SiF}_{4}$.

The code could now proceed to a continuation problem as specified by the CNTN keyword or to a second PSR in series with the first, specified by an NPSR keyword. However, in the interest of keeping this manual to a reasonable length, we have omitted an example of these features.

In this example we used a lot of printing in order to explain each of the various outputs. However, when problems are running without difficulty, we recommend using PRNT 0 . This provides an uncluttered printed output that contains only the solutions. 


\section{CHEMKIN Input for the $\mathrm{Si}_{3} \mathrm{~N}_{4}$ Mechanism}

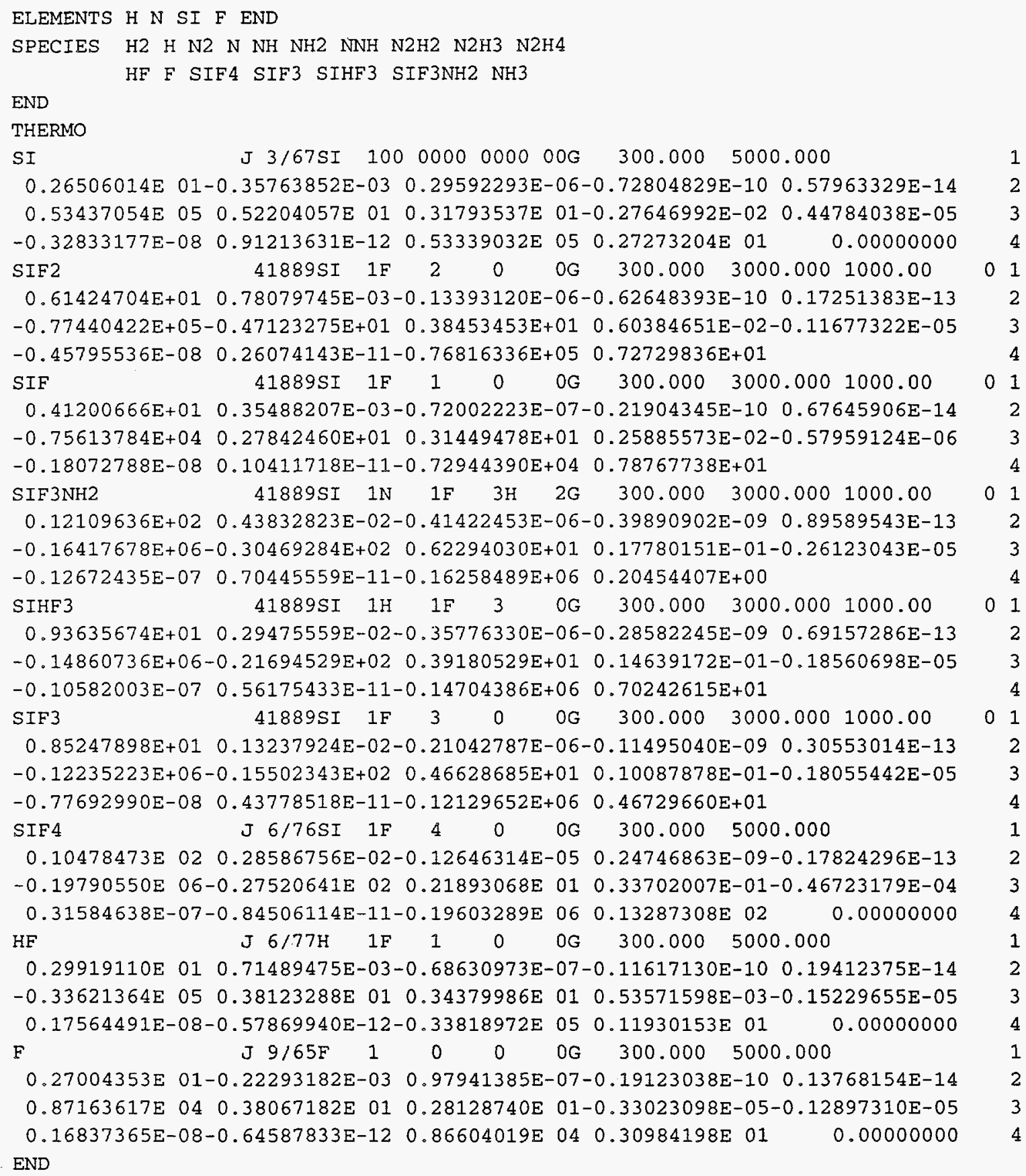


REACTIONS

$\mathrm{H}+\mathrm{H}+\mathrm{M}=\mathrm{H} 2+\mathrm{M}$

$\mathrm{H} 2 / 0.0 /$

$\mathrm{H}+\mathrm{H}+\mathrm{H} 2=\mathrm{H} 2+\mathrm{H} 2$

$\mathrm{NH}+\mathrm{N}=\mathrm{N} 2+\mathrm{H}$

$\mathrm{NH}+\mathrm{H}=\mathrm{N}+\mathrm{H} 2$

$\mathrm{NH} 2+\mathrm{H}=\mathrm{NH}+\mathrm{H} 2$

$\mathrm{NH} 3+\mathrm{H}=\mathrm{NH} 2+\mathrm{H} 2$

$\mathrm{NNH}=\mathrm{N} 2+\mathrm{H}$

$\mathrm{NNH}+\mathrm{H}=\mathrm{N} 2+\mathrm{H} 2$

$\mathrm{NNH}+\mathrm{NH} 2=\mathrm{N} 2+\mathrm{NH} 3$

$\mathrm{NNH}+\mathrm{NH}=\mathrm{N} 2+\mathrm{NH} 2$

$\mathrm{NH} 2+\mathrm{NH}=\mathrm{N} 2 \mathrm{H} 2+\mathrm{H}$

$\mathrm{NH}+\mathrm{NH}=\mathrm{N} 2+\mathrm{H}+\mathrm{H}$

$\mathrm{NH} 2+\mathrm{N}=\mathrm{N} 2+\mathrm{H}+\mathrm{H}$

$\mathrm{N} 2 \mathrm{H} 2+\mathrm{M}=\mathrm{NNH}+\mathrm{H}+\mathrm{M}$

$\mathrm{N} 2 / 2 / \mathrm{H} 2 / 2 /$

$\mathrm{N} 2 \mathrm{H} 2+\mathrm{H}=\mathrm{NNH}+\mathrm{H} 2$

$\mathrm{N} 2 \mathrm{H} 2+\mathrm{NH}=\mathrm{NNH}+\mathrm{NH} 2$

$\mathrm{N} 2 \mathrm{H} 2+\mathrm{NH} 2=\mathrm{NH} 3+\mathrm{NNH}$

$\mathrm{NH} 2+\mathrm{NH} 2=\mathrm{N} 2 \mathrm{H} 2+\mathrm{H} 2$

$\mathrm{NH} 3+\mathrm{M}=\mathrm{NH} 2+\mathrm{H}+\mathrm{M}$

$\mathrm{N} 2 \mathrm{H} 3+\mathrm{H}=\mathrm{NH} 2+\mathrm{NH} 2$

$\mathrm{N} 2 \mathrm{H} 3+\mathrm{M}=\mathrm{N} 2 \mathrm{H} 2+\mathrm{H}+\mathrm{M}$

$\mathrm{N} 2 \mathrm{H} 3+\mathrm{NH}=\mathrm{NH} 2+\mathrm{N} 2 \mathrm{H} 2$

$\mathrm{NH} 2+\mathrm{NH} 2+\mathrm{M}=\mathrm{N} 2 \mathrm{H} 4+\mathrm{M}$

$\mathrm{H}+\mathrm{N} 2 \mathrm{H} 4=\mathrm{H} 2+\mathrm{N} 2 \mathrm{H} 3$

$\mathrm{NH} 2+\mathrm{N} 2 \mathrm{H} 4=\mathrm{NH} 3+\mathrm{N} 2 \mathrm{H} 3$

$\mathrm{NH}+\mathrm{H}+\mathrm{M}=\mathrm{NH} 2+\mathrm{M}$

$\mathrm{NH} 2+\mathrm{NH} 2=\mathrm{NH} 3+\mathrm{NH}$

$\mathrm{F}+\mathrm{NH} 3=\mathrm{NH} 2+\mathrm{HF}$

SIF $4=S I F 3+F$

$\mathrm{H}+\mathrm{SIF} 4=\mathrm{HF}+\mathrm{SIF} 3$

$\mathrm{NH} 2+\mathrm{SIF} 4=\mathrm{SIF} 3 \mathrm{NH} 2+\mathrm{F}$

$\mathrm{NH} 3+\mathrm{SIF} 3=\mathrm{SIF} 3 \mathrm{NH} 2+\mathrm{H}$

$\mathrm{NH} 3+\mathrm{SIF} 3=\mathrm{SIHF} 3+\mathrm{NH} 2$

END

\begin{tabular}{|c|c|c|c|c|}
\hline $0.100 E+19$ & -1.000 & 0.000 & ! & $D-L$ \\
\hline $0.920 E+17$ & -0.600 & 0.000 & & \\
\hline $0.300 \mathrm{E}+14$ & 0.000 & 0.000 & $!$ & JAM \\
\hline $0.100 E+15$ & 0.000 & 0.000 & $!$ & NH3 \\
\hline $0.692 E+14$ & 0.000 & 3650.000 & & \\
\hline $0.636 \mathrm{E}+06$ & 2.390 & 10171.000 & $!$ & MICHAEL \\
\hline $0.100 E+05$ & 0.000 & 0.000 & $!$ & JAM \\
\hline $0.100 E+15$ & 0.000 & 0.000 & $!$ & JAM \\
\hline $0.500 \mathrm{E}+14$ & 0.000 & 0.000 & $!$ & JAM \\
\hline $0.500 E+14$ & 0.000 & 0.000 & $!$ & JAM \\
\hline $0.500 E+14$ & 0.000 & 0.000 & $!$ & NH3CST \\
\hline $0.254 \mathrm{E}+14$ & 0.000 & 0.000 & $!$ & NH3 CST \\
\hline $0.720 E+14$ & 0.000 & 0.000 & $!$ & PG \\
\hline $0.500 E+17$ & 0.000 & 50000.000 & $!$ & NH3 CST \\
\hline $0.500 E+14$ & 0.000 & 1000.000 & $!$ & NH3 CST \\
\hline $0.100 \mathrm{E}+14$ & 0.000 & 1000.000 & $!$ & NH3 CST \\
\hline $0.100 \mathrm{E}+14$ & 0.000 & 1000.000 & $!$ & NH3 CST \\
\hline $0.500 \mathrm{E}+12$ & 0.000 & 0.000 & $!$ & NH3 CST \\
\hline $0.140 \mathrm{E}+17$ & 0.000 & 90600.000 & $!$ & MSGK \\
\hline 1. $60 \mathrm{E}+12$ & 0.0 & 0.0 & $!$ & MSGK \\
\hline $3.50 \mathrm{E}+16$ & 0.0 & 46000.0 & $!$ & MSGK \\
\hline $2.00 E+13$ & 0.0 & 0.0 & $!$ & MSGK \\
\hline $3.00 \mathrm{E}+20$ & -1.0 & 0.0 & $!$ & MSGK \\
\hline $1.30 E+13$ & 0.0 & 2500.0 & $!$ & MSGK \\
\hline $3.90 \mathrm{E}+12$ & 0.0 & 1500.0 & $!$ & MSGK \\
\hline $2.00 \mathrm{E}+16$ & -0.5 & 0.0 & $!$ & MSGK \\
\hline $5.00 E+12$ & 0.0 & 10000.0 & $!$ & MSGK \\
\hline $4.27 E+11$ & 0.5 & 800.0 & $!$ & KONDRATIEV \\
\hline $3.00 \mathrm{E}+12$ & 0.0 & 147170.0 & $!$ & PHO\&MEC \\
\hline $1.00 \mathrm{E}+13$ & 0.0 & 50000.0 & $!$ & PHO\&MEC \\
\hline $1.00 \mathrm{E}+11$ & 0.0 & 40950.0 & $!$ & GUESS \\
\hline $1.00 \mathrm{E}+11$ & 0.0 & 5000.0 & $!$ & GUESS \\
\hline $1.00 \mathrm{E}+11$ & 0.0 & 10000.0 & $!$ & PHO\&MEC \\
\hline
\end{tabular}




\section{SURFACE CHEMKIN Input for the $\mathrm{Si}_{3} \mathrm{~N}_{4}$ Mechanism}

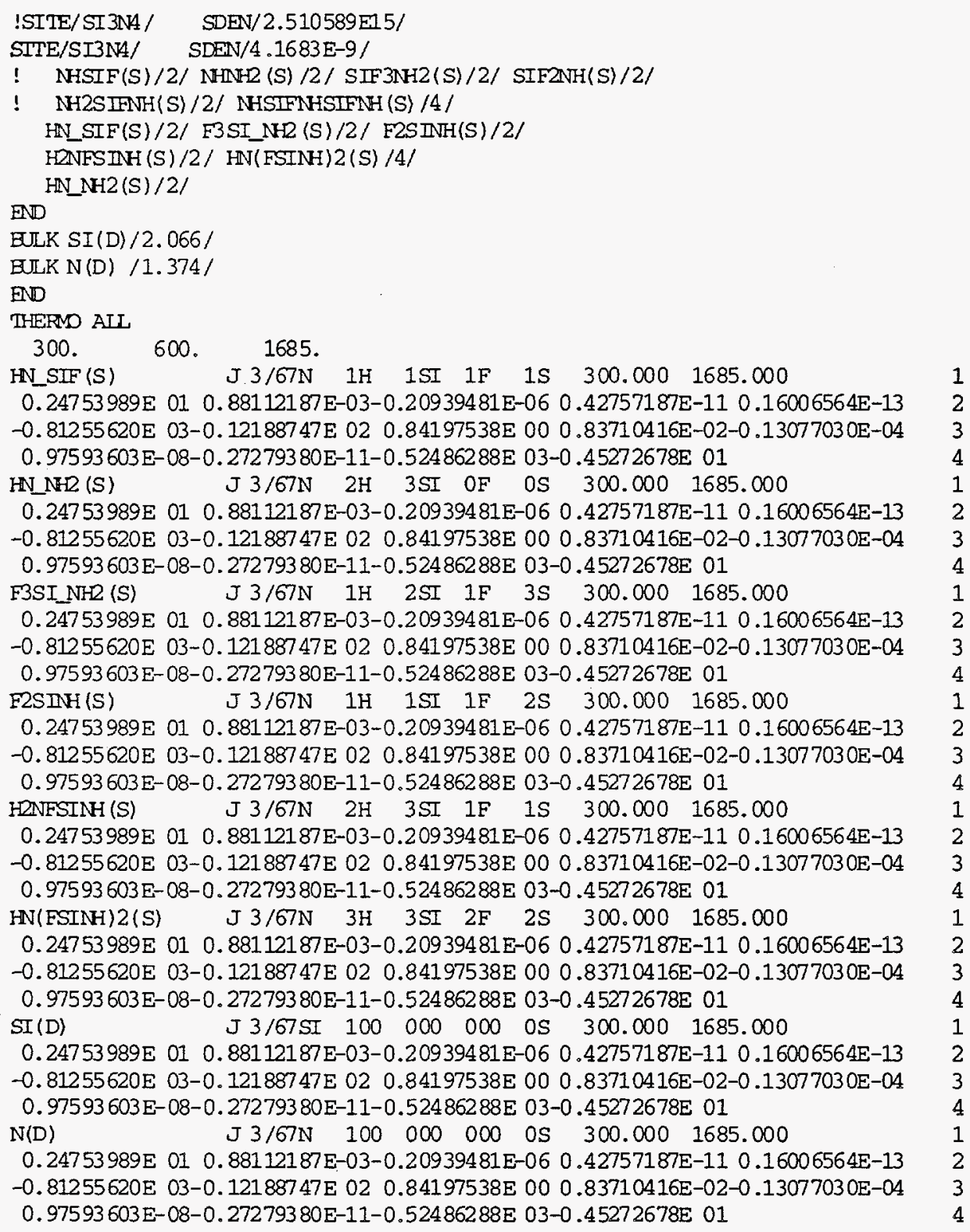

END

\begin{tabular}{|c|c|c|c|c|c|}
\hline \multicolumn{6}{|l|}{ REACIIONS } \\
\hline $\mathrm{NH} 3$ & + HN_SIF (S) & $\Rightarrow$ HN_NE2 $(S)$ & $+S I(D)$ & $+M F$ & $7.562 \mathrm{F0} 8$ \\
\hline SIF4 & $+\mathrm{HN} N \mathrm{NH} 2(\mathrm{~S})$ & $\Rightarrow$ F3SI_NH2(S) & $+N(D)$ & $+H F$ & $3.0967 \mathrm{E} 8$ \\
\hline F3SI_NH2 (S) & & $\Rightarrow$ F2SINH(S) & & $+\mathrm{HF}$ & $\begin{array}{lll}1.0 \mathrm{E} 05 & 0.0 & 0.0\end{array}$ \\
\hline $\mathrm{NH} 3$ & $+F 2 \operatorname{SINH}(S)$ & $\Rightarrow H 2 N E \operatorname{SINH}(S)$ & & $+\mathrm{HF}$ & $7.562 \mathrm{E0} 8$ \\
\hline HZNESINH(S) & $+F 2 S \operatorname{INH}(S)$ & $\Rightarrow H N(F S \mathbb{N} H) 2(S$ & & $+\mathrm{HF}$ & $\begin{array}{lll}1.0 \mathrm{E} 15 & 0.0 & 0.0\end{array}$ \\
\hline $\mathrm{HN}$ (FSINH) 2 (S) & $+F 2 \operatorname{SINH}(S)$ & $\Rightarrow$ 3HN_SIF $(\mathrm{S})$ & $+N(D)$ & $+\mathrm{HF}$ & 0.00 .0 \\
\hline$E \mathbb{D}$ & & & & & \\
\hline
\end{tabular}




\title{
AURORA Output for the $\mathrm{Si}_{3} \mathrm{~N}_{4}$ Example
}

\author{
PSR: PERFECTLY STIRRED REACTOR CODE \\ VERSION $4.5,95 / 12 / 14$ \\ DOBLE PRECISION
}

CKIIB: Chemical Kinetics Library

CHEMIIN-III Version 4.7, August 1995

DOUBIE PRECISION

SKIIB: Surface kinetics library

Copyright 1990, Sandia Corporation.

The U.S. Goverment retains a limited license in this software. CHEMMIN-III Version 5.5, August 1995

DOUBLE PRECISION

$\begin{array}{lrc} & \text { WORKING SPACE REQTIREMENTS } \\ & \text { PROVIIED } & \text { REQUIRED } \\ \text { IOGICAL } & 1000 & 126 \\ \text { INTEGER } & 6500 & 2602 \\ \text { REAL } & 26000 & 7010 \\ \text { CHARACTER } & 1800 & 90\end{array}$

KEYWORD INEUT

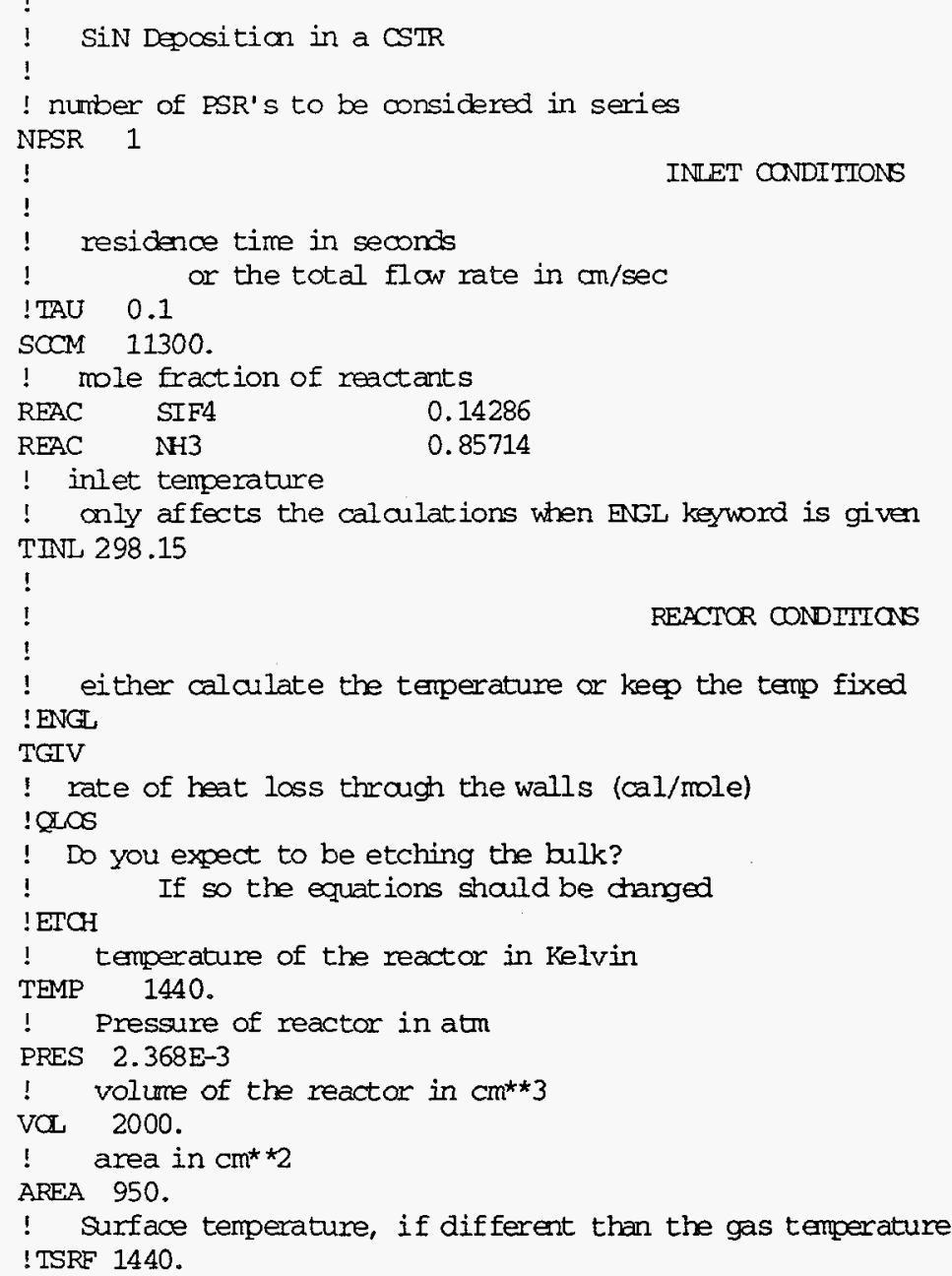




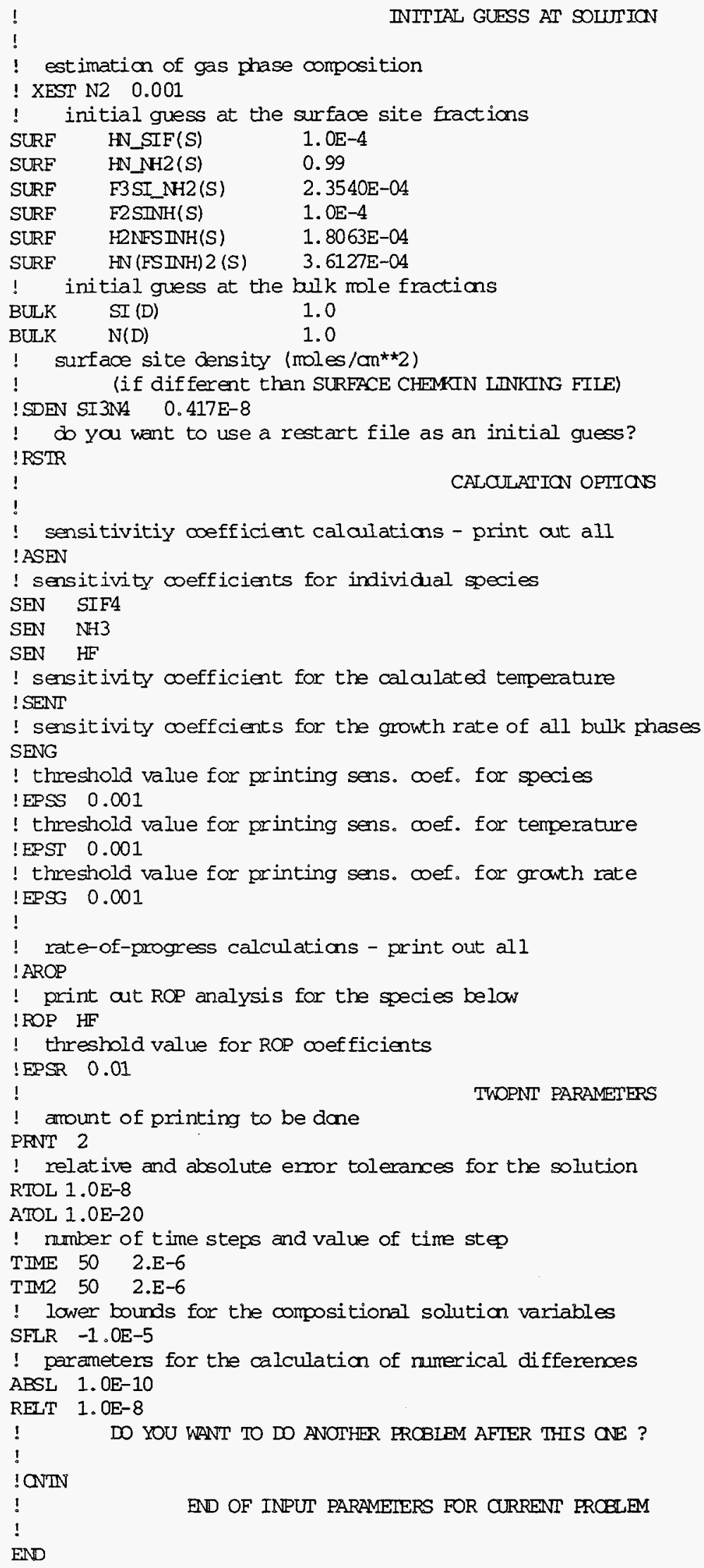


WARNING... "TINL" SPECIFIED FOR "TGIV" PROBLEM

CAUTION. .XEST MOTE FRACTIONS SUM TO $0.9909772999999999 \quad$ FCR PHASE $2=S I 3 N 4$

EQUIL: Chemkin interface for Stanjan-III CHEMKIN-II Version 3.0, Decerber 1992 DOUBLE PRECISTON

\begin{tabular}{lrr}
\multicolumn{4}{c}{ WORKIIN SPACE REQUIREMENTS } \\
EROVIDED & REQUIRED \\
TNTEGER & 456 & 456 \\
REAL & 1120 & 1120 \\
STIANJAN: & Version 3.8C, MaY 1988 &
\end{tabular}

w. C. Reynolds, Stanford univ.

\section{FIRST SOLUTION ESTIMATE IS EQUIIIBRIM}

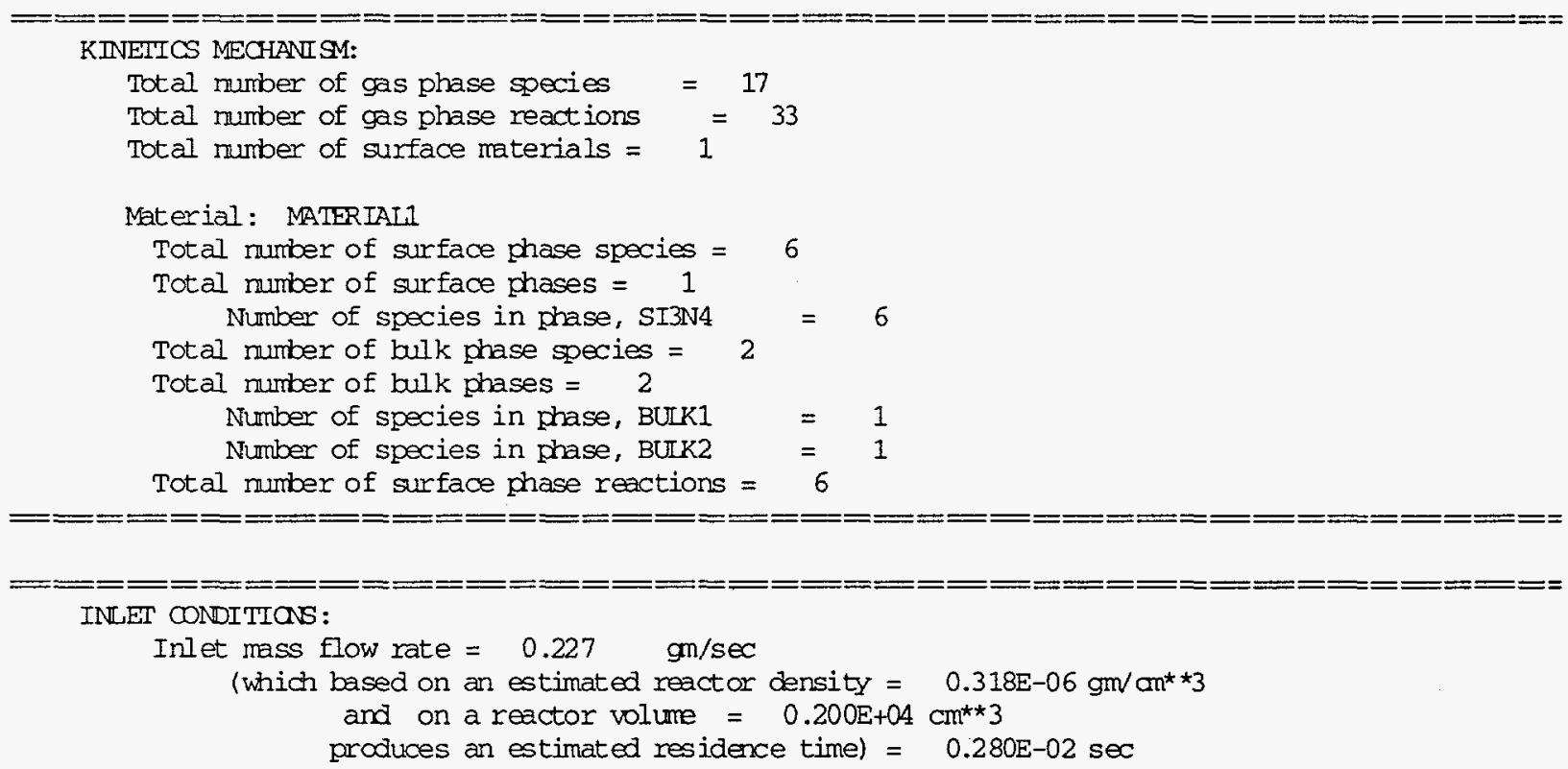

Inlet temperature $=298.15$ Kelvin

Inlet pressure (assumed equal to reactor pressure) $=0.237 \mathrm{E}-02 \mathrm{~atm}$

Inlet density $=0.28520 \mathrm{E}-05 \mathrm{gm} / \mathrm{am}^{\star \star} 3$

Inlet mean molecular weight $=29.466 \mathrm{gm} / \mathrm{mole}$

Inlet molar flow rate $\quad=0.76979 \mathrm{E}-02 \mathrm{moles} / \mathrm{sec}$

Inlet volumetric flow rate $=79533$. $\mathrm{cm}^{\star *} 3 / \mathrm{sec}$

(based on reactor pressure and inlet temperature)

$$
\begin{array}{ll}
=11300 . & \text { SCCM } \\
=11.300 & \text { SLPM }
\end{array}
$$

INLET CONDITIONS FOR GAS PHASE MOLECUIAR SPECIES:

\begin{tabular}{lcccccc} 
Species & moleffrac & moles/sec & $\mathrm{gm} / \mathrm{sec}$ & $\mathrm{cm} * * 3 / \mathrm{sec}$ & $\mathrm{s} 0 \mathrm{CM}$ & SLAM \\
\hline H2 & $0.00000 \mathrm{E}+00$ & $0.00000 \mathrm{E}+00$ & $0.00000 \mathrm{E}+00$ & $0.00000 \mathrm{E}+00$ & $0.00000 \mathrm{E}+00$ & $0.00000 \mathrm{E}+00$ \\
H & $0.00000 \mathrm{E}+00$ & $0.00000 \mathrm{E}+00$ & $0.00000 \mathrm{E}+00$ & $0.00000 \mathrm{E}+00$ & $0.00000 \mathrm{E}+00$ & $0.00000 \mathrm{E}+00$ \\
NR & $0.00000 \mathrm{E}+00$ & $0.00000 \mathrm{E}+00$ & $0.00000 \mathrm{E}+00$ & $0.00000 \mathrm{E}+00$ & $0.00000 \mathrm{E}+00$ & $0.00000 \mathrm{E}+00$ \\
N & $0.00000 \mathrm{E}+00$ & $0.00000 \mathrm{E}+00$ & $0.00000 \mathrm{E}+00$ & $0.00000 \mathrm{E}+00$ & $0.00000 \mathrm{E}+00$ & $0.00000 \mathrm{E}+00$ \\
NH & $0.00000 \mathrm{E}+00$ & $0.00000 \mathrm{E}+00$ & $0.00000 \mathrm{E}+00$ & $0.00000 \mathrm{E}+00$ & $0.00000 \mathrm{E}+00$ & $0.00000 \mathrm{E}+00$ \\
NH2 & $0.00000 \mathrm{E}+00$ & $0.00000 \mathrm{E}+00$ & $0.00000 \mathrm{E}+00$ & $0.00000 \mathrm{E}+00$ & $0.00000 \mathrm{E}+00$ & $0.00000 \mathrm{E}+00$ \\
NNH & $0.00000 \mathrm{E}+00$ & $0.00000 \mathrm{E}+00$ & $0.00000 \mathrm{E}+00$ & $0.00000 \mathrm{E}+00$ & $0.00000 \mathrm{E}+00$ & $0.00000 \mathrm{E}+00$ \\
NRH2 & $0.00000 \mathrm{E}+00$ & $0.00000 \mathrm{E}+00$ & $0.00000 \mathrm{E}+00$ & $0.00000 \mathrm{E}+00$ & $0.00000 \mathrm{E}+00$ & $0.00000 \mathrm{E}+00$
\end{tabular}




$\begin{array}{lllllll}\mathrm{N} H 3 & 0.00000 \mathrm{E}+00 & 0.00000 \mathrm{E}+00 & 0.00000 \mathrm{E}+00 & 0.00000 \mathrm{E}+00 & 0.00000 \mathrm{E}+00 & 0.00000 \mathrm{E}+00 \\ \mathrm{NRH4} & 0.00000 \mathrm{E}+00 & 0.00000 \mathrm{E}+00 & 0.00000 \mathrm{E}+00 & 0.00000 \mathrm{E}+00 & 0.00000 \mathrm{E}+00 & 0.00000 \mathrm{E}+00 \\ \mathrm{HF} & 0.00000 \mathrm{E}+00 & 0.00000 \mathrm{E}+00 & 0.00000 \mathrm{E}+00 & 0.00000 \mathrm{E}+00 & 0.00000 \mathrm{E}+00 & 0.00000 \mathrm{E}+00 \\ \mathrm{~F} & 0.00000 \mathrm{E}+00 & 0.00000 \mathrm{E}+00 & 0.00000 \mathrm{E}+00 & 0.00000 \mathrm{E}+00 & 0.00000 \mathrm{E}+00 & 0.00000 \mathrm{E}+00 \\ \text { SIF4 } & 0.14286 & 0.10997 \mathrm{E}-02 & 0.11446 & 11362 \mathrm{E} & 1614.3 & 1.6143 \\ \text { SIF3 } & 0.00000 \mathrm{E}+00 & 0.00000 \mathrm{E}+00 & 0.00000 \mathrm{E}+00 & 0.00000 \mathrm{E}+00 & 0.00000 \mathrm{E}+00 & 0.0000 \mathrm{E}+00 \\ \text { SIHF3 } & 0.00000 \mathrm{E}+00 & 0.00000 \mathrm{E}+00 & 0.00000 \mathrm{E}+\infty & 0.00000 \mathrm{E}+00 & 0.00000 \mathrm{E}+00 & 0.00000 \mathrm{E}+00 \\ \text { SIF3NH2 } & 0.00000 \mathrm{E}+00 & 0.00000 \mathrm{E}+00 & 0.00000 \mathrm{E}+00 & 0.00000 \mathrm{E}+00 & 0.00000 \mathrm{E}+00 & 0.00000 \mathrm{E}+00 \\ \text { NH3 } & 0.85714 & 0.65982 \mathrm{E}-02 & 0.11237 & 68171 . & 9685.7 & 9.6857\end{array}$

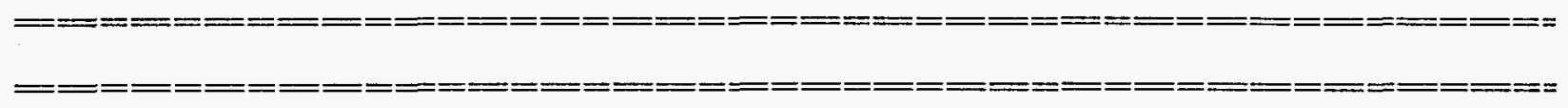

TWOPNT PARAMETERS:

PRINIING: Level(1) = 2 Level $(2)=2$

TWOPN subroutines 1 below entrance will all write to output

SOUTION BOUNDS FCR VARIABLES:

\begin{tabular}{|c|c|c|}
\hline Variable & Upper_bound & Lower bound \\
\hline TEMPERATURE & $1.000 \mathrm{E}+04$ & 200 \\
\hline $\mathrm{H} 2$ & 1.01 & $-1.000 \mathrm{E}-05$ \\
\hline $\mathrm{H}$ & 1.01 & $-1.000 \mathrm{E}-05$ \\
\hline $\mathrm{N} 2$ & 1.01 & $-1.000 \mathrm{E}-05$ \\
\hline $\mathrm{N}$ & 1.01 & $-1.000 \mathrm{E}-05$ \\
\hline $\mathrm{NH}$ & 1.01 & $-1.000 \mathrm{E}-05$ \\
\hline $\mathrm{NH} 2$ & 1.01 & $-1.000 \mathrm{E}-05$ \\
\hline $\mathrm{NNH}$ & 1.01 & $-1.000 E-05$ \\
\hline NDH2 & 1.01 & $-1.000 E-05$ \\
\hline $\mathbb{N} 213$ & 1.01 & $-1.000 E-05$ \\
\hline $\mathrm{N} 2 \mathrm{H} 4$ & 1.01 & $-1.000 \mathrm{E}-05$ \\
\hline $\mathrm{HF}$ & 1.01 & $-1.000 E-05$ \\
\hline$F$ & 1.01 & $-1.000 E-05$ \\
\hline SIF4 & 1.01 & $-1.000 E-05$ \\
\hline SIF3 & 1.01 & $-1.000 \mathrm{E}-05$ \\
\hline SIHE3 & 1.01 & $-1.000 E-05$ \\
\hline SIF3NH2 & 1.01 & $-1.000 E-05$ \\
\hline $\mathrm{NH3}$ & 1.01 & $-1.000 E-05$ \\
\hline $\mathrm{HN}$ SIF $(S)$ & 1.01 & $-1.000 E-05$ \\
\hline F3SI_NH2 (S) & 1.01 & $-1.000 E-05$ \\
\hline F2SINH(S) & 1.01 & $-1.000 \mathrm{E}-05$ \\
\hline $\mathrm{H} 2 \mathrm{NFSTNH}(\mathrm{S})$ & 1.01 & $-1.000 E-05$ \\
\hline HN (FSINH) 2 ( & 1.01 & $-1.000 E-05$ \\
\hline $\mathrm{HN} N \mathrm{H} 2(\mathrm{~S})$ & 1.01 & $-1.000 \mathrm{E}-05$ \\
\hline$S I \overline{(D)}$ & 1.01 & $-1.000 E-05$ \\
\hline$N(D)$ & 1.01 & $-1.000 E-05$ \\
\hline
\end{tabular}

Maxinum number of time steps to try if newton's method fails $=50$

Absolute bound for the steady-state problem $=1.000 \mathrm{E}-20$

Relative bound for the steady-state problem $=1.000 \mathrm{E}-08$

Absolute bound for the time-dependent prob $=1.000 \mathrm{E}-09$

Relative bound for the time-dependent prob $=1.000 \mathrm{E}-04$

Number of steps to be taken before increasing the time step $=25$

Factor by which to decrease time-step when necessary $=2.20$

Factor by which to increase time-step when necessary $=2.00$

Mininum time step allowed $=1.000 \mathrm{E}-10$

Initial time step $=2.000 \mathrm{E}-06$

Absolute delta for numerical differencing $=1.000 \mathrm{E}-10$

Relative delta for numerical differencing $=1.000 \mathrm{E}-08$ 
TWOANT: DOUBLE PRECISION (TWD POINT BOUNDARY VAUE PROBLEM) SOLVER, VERSION 3.18 OF DECEMRER 1995 BY DR. JOSEPH F. GRCAR.

TWOANT: INITIAL GUESS:

PSERNT: Printing of current solution from TWOANT:

$\begin{array}{lcl}\text { RESIDENCE TIME } & 0.2803 \mathrm{E}-02 & \text { SEC } \\ \text { MASS FLON RATE } & 0.2268 \mathrm{E}+00 & \mathrm{GM} / \mathrm{SEC} \\ \text { PRESSURE } & 0.2368 \mathrm{E}-02 & \text { ATM } \\ \text { MASS DENSITY } & 0.3179 \mathrm{E}-06 & \mathrm{GM} / \mathrm{CMB} \\ \text { VOLIME } & 2000 . & \mathrm{CM} \\ \text { SURFACE AREA } & 950.0 & \mathrm{CM} \\ \text { SURFACE TO VOLIME RATIO } & 0.4750 & \mathrm{CM}-1 \\ \text { TEMPERATURE (FIXED) } & 1440 . & \mathrm{K}\end{array}$

INLET GAS RHASE MOIE FRACTIONS

\begin{tabular}{|c|c|c|c|c|c|c|}
\hline$H 2$ & $=0.0000 \mathrm{E}+00$ & $\mathrm{H}$ & $=0.0000 \mathrm{E}+00$ & $\mathrm{~N} 2$ & $=$ & $0.0000 \mathrm{E}+00$ \\
\hline $\mathrm{N}$ & $0.0000 \mathrm{E}+00$ & $\mathrm{NH}$ & $0.0000 \mathrm{E}+00$ & $\mathrm{NH} 2$ & $=$ & $0.0000 \mathrm{E}+00$ \\
\hline $\mathrm{NNH}$ & $0.0000 \mathrm{E}+00$ & $\mathrm{~N} 2 \mathrm{H} 2$ & $0.0000 E+00$ & $\mathrm{~N} 2 \mathrm{HB}$ & $=$ & $0.0000 \mathrm{E}+00$ \\
\hline $\mathrm{N} \mathrm{NH} 4$ & $=0.0000 \mathrm{E}+00$. & $\mathrm{HF}$ & $0.0000 \mathrm{E}+00$ & F & $=$ & $0.0000 \mathrm{E}+00$ \\
\hline SIF4 & $=0.1429$ & SIF3 & $0.0000 \mathrm{E}+00$ & SIHF3 & $=$ & $0.0000 \mathrm{E}+00$ \\
\hline STF3NH2 & $=0.0000 \mathrm{E}+00$ & NHB & 0.8571 & & & \\
\hline
\end{tabular}

\section{EXIT GAS PHASE MOTE FRACTIONS}

$\begin{array}{llllll}\mathrm{H} 2 & =0.6861 & \mathrm{H} & =0.1407 \mathrm{E}-03 & \mathrm{~N} 2 & =0.2308 \\ \mathrm{~N} & =0.1206 \mathrm{E}-12 & \mathrm{NH} & =0.5163 \mathrm{E}-12 & \mathrm{NH} & =0.2122 \mathrm{E}-10 \\ \mathrm{NNH} & =0.1598 \mathrm{E}-12 & \mathrm{~N} 2 \mathrm{H} 2 & =0.8936 \mathrm{E}-17 & \mathrm{~N} 2 \mathrm{HB} & =0.5304 \mathrm{E}-19 \\ \mathrm{~N} H 4 & =0.3072 \mathrm{E}-21 & \mathrm{HF} & =0.6052 \mathrm{E}-02 & \mathrm{~F} & =0.2253 \mathrm{E}-10 \\ \text { SIF4 } & =0.7087 \mathrm{E}-01 & \text { SIF3 } & =0.1590 \mathrm{E}-04 & \text { SIHF3 } & =0.6035 \mathrm{E}-02 \\ \text { SIF3NH2 } & =0.8323 \mathrm{E}-06 & \mathrm{NHB} & & =0.4947 \mathrm{E}-07 & \end{array}$

SURFACE SITE FRACTIONS IN SURFACE PHASE, SI 3NA

Site density $=0.4168 \mathrm{E}-08 \mathrm{~mole} / \mathrm{cm}^{\star \star 2}$

Standard State Site density $=0.4168 \mathrm{E}-08 \mathrm{~mole} / \mathrm{cm}^{*}{ }^{*}$

Rate of change of site density $=0.0000 \mathrm{E}+00 \mathrm{~mole} /(\mathrm{cm} \star * 2 * \mathrm{sec})$

$\begin{array}{llllll}\text { HN_SIF(S) } & =0.1009 \mathrm{E}-03 & \text { F3SI_NH2(S) } & =0.2375 \mathrm{E}-03 & \text { F2SINH (S) } & =0.1009 \mathrm{E}-03 \\ \text { H2NFSINH(S) } & =0.1823 \mathrm{E}-03 & \text { HN(FSINH) 2(S) } & =0.3646 \mathrm{E}-03 & \text { HN NH2 (S) } & =0.9990\end{array}$

BULK PHASE MOLE FRACTIONS AND ACTIVITIES IN BULK PHASE, BULKI

Total growth rate of bulk phase $=0.8133 \mathrm{E}-16 \mathrm{~cm} / \mathrm{sec}$

Density of the bulk phase $=2.066 \mathrm{gm} / \mathrm{cm}^{\star} \star 3$

$=0.1596 \mathrm{E}-12 \mathrm{gm} / \mathrm{sec}$

Average moleaular weight of bulk phase $=28.09 \mathrm{gm} / \mathrm{mole}$

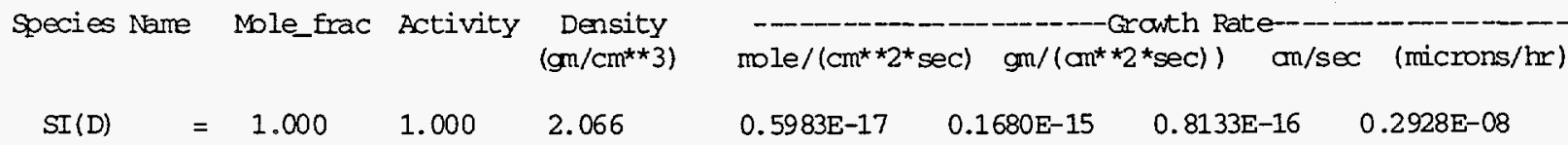


BULK PHASE MOLE FRACTIONS AND ACTIVITIES IN BUIK PHASE, BUL2

Total growth rate of bulk phase $=0.3550 \mathrm{E}-06 \mathrm{~cm} / \mathrm{sec}$

Density of the bulk phase $=1.374 \mathrm{gm} / \mathrm{cm}^{* * 3}$

$=0.4634 \mathrm{E}-03 \mathrm{gm} / \mathrm{sec}$

Average molecular weight of bulk phase $=14.01 \mathrm{gm} / \mathrm{mole}$

Species Name Mole_frac Activity Density $\left(\mathrm{gm} / \mathrm{cm}^{\star *} 3\right)$ $\left.\mathrm{mole} /\left(\mathrm{cm}^{\star \star} 2 * \mathrm{sec}\right) \mathrm{gm} /\left(\mathrm{cm}^{\star *} 2 * \mathrm{sec}\right)\right) \mathrm{cm} / \mathrm{sec}$ (microns/hr)

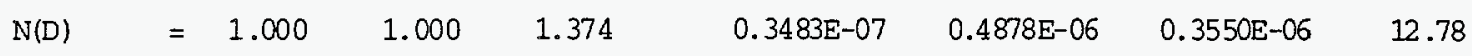

PRINTOUT OF RAN RESIDLALS:

$\begin{array}{llc}\text { Temperature } & : & 0.000 \mathrm{E}+00 \\ \mathrm{H} 2 & : & -30.8 \\ \mathrm{H} & : & -0.316 \mathrm{E}-02 \\ \mathrm{~N} 2 & : & -144 . \\ \mathrm{N} & : & -0.377 \mathrm{E}-10 \\ \mathrm{NH} & : & -0.173 \mathrm{E}-09 \\ \mathrm{NH2} & : & -0.758 \mathrm{E}-08 \\ \mathrm{NNH} & : & -0.102 \mathrm{E}-09 \\ \mathrm{~N} 2 \mathrm{H} 2 & : & -0.598 \mathrm{E}-14 \\ \mathrm{~N} 2 \mathrm{H} 3 & : & -0.367 \mathrm{E}-16 \\ \mathrm{~N} 2 \mathrm{H} 4 & : & -0.220 \mathrm{E}-18 \\ \mathrm{HF} & : & -0.177 \\ \text { F } & : & -0.955 \mathrm{E}-08 \\ \text { SIF4 } & : & 10.1 \\ \text { SIF3 } & : & -0.302 \mathrm{E}-01 \\ \text { SIHF3 } & : & -11.6 \\ \text { SIF3NH2 } & : & -0.188 \mathrm{E}-02 \\ \text { NH3 } & : & 177 .\end{array}$

Surface phase site fraction residuals:

HN_SIF (S) : 0.115

F3SI_NH2 (S) : $\quad-7.08$

F2SINH (S) : $\quad 23.7$

H2NFSINH (S) : $\quad-0.383 \mathrm{E}-01$

$\mathrm{HN}($ FSINH) 2 (S) : $-0.212 \mathrm{E}-05$

HN_NE (S) : -16.7

Bulk phase mole fraction residuals:

SI (D) $\quad: \quad 0.000 \mathrm{E}+00$

$\mathrm{N}(\mathrm{D}) \quad: \quad 0.000 \mathrm{E}+00$

TWORNT: CALLTNG SEAROH TO SOLVE THE STEADY STATE PROBLEM.

SEARCH: SOLVE NONIINEAR, NONDIFFERENTIAL EQUATIONS.

\begin{tabular}{|c|c|c|c|c|c|c|c|}
\hline \multirow{2}{*}{$\begin{array}{r}\text { SLIN } \\
\text { NUMBER }\end{array}$} & \multicolumn{7}{|l|}{ LOG10 } \\
\hline & NORM $F$ & CAND J & NORM S & $\mathrm{ABS}$ & D REL & DELTA & AND D \\
\hline 0 & 2.25 & 8.37 & 3.60 & 3.60 & 7.60 & -6.94 & -0.30 \\
\hline 1 & 2.25 & 7.65 & 2.49 & 2.49 & 6.84 & -6.21 & \\
\hline 2 & 2.25 & 6.70 & 2.44 & 2.44 & 6.42 & -6.10 & \\
\hline 3 & 2.25 & 6.55 & 0.68 & 0.68 & 6.14 & ZERO & \\
\hline
\end{tabular}

SEARCH: FAILURE. THE SEARCH FOR THE FOLLOWING UNRNOWN GOES OUI OF BOUNDS.
BOTD VALUE UNKNONN
LONER $-1.00 \mathrm{E}-05 \quad$ (C $20 \mathrm{P} 1)$ 
TWOANT: SEARCH DID NOT FIND THE STEADY STATE.

TWOANT: CALLMNG ENOLVE TO PERFCRM TIME EVUUTION.

EVOLVE: BEGIN TIME EVOLUTION.

\begin{tabular}{|c|c|c|c|c|c|c|c|}
\hline TIME & IOG10 & & & NEWION & SEAR & & \\
\hline NLMBER & NORM F & CHANGE & STRIDE & STEPS & J'S & COND J & REMAPK \\
\hline 0 & 2.25 & & & & & & \\
\hline 1 & 2.25 & -3.45 & -5.70 & 1 & 1 & 0.04 & \\
\hline 2 & 2.25 & -3.45 & -5.70 & 1 & & & \\
\hline 3 & 2.25 & -3.45 & -5.70 & 1 & & & \\
\hline 4 & 2.25 & -3.45 & -5.70 & 1 & & & \\
\hline 5 & 2.25 & -3.45 & -5.70 & 1 & & & \\
\hline 6 & 2.24 & -3.45 & -5.70 & 1 & & & \\
\hline 7 & 2.24 & -3.45 & -5.70 & 1 & & & \\
\hline 8 & 2.24 & -3.46 & -5.70 & 1 & & & \\
\hline 9 & 2.24 & -3.46 & -5.70 & 1 & & & \\
\hline 10 & 2.24 & -3.46 & -5.70 & 1 & & & \\
\hline 11 & 2.24 & -3.46 & -5.70 & 1 & & & \\
\hline 12 & 2.24 & -3.46 & -5.70 & 1 & & & \\
\hline 13 & 2.24 & -3.46 & -5.70 & 1 & & & \\
\hline 14 & 2.24 & -3.46 & -5.70 & 1 & & & \\
\hline 15 & 2.24 & -3.46 & -5.70 & 1 & & & \\
\hline 16 & 2.24 & -3.46 & -5.70 & 1 & & & \\
\hline 17 & 2.24 & -3.46 & -5.70 & 1 & & & \\
\hline 18 & 2.24 & -3.46 & -5.70 & 1 & & & \\
\hline 19 & 2.24 & -3.46 & -5.70 & 1 & & & \\
\hline 20 & 2.24 & -3.46 & -5.70 & 1 & & & \\
\hline 21 & 2.24 & -3.46 & -5.70 & 1 & 1 & 0.04 & \\
\hline 22 & 2.24 & -3.46 & -5.70 & 1 & & & \\
\hline 23 & 2.24 & -3.46 & -5.70 & 1 & & & \\
\hline 24 & 2.24 & -3.46 & -5.70 & 1 & & & \\
\hline 25 & 2.24 & -3.46 & -5.70 & 1 & & & \\
\hline 26 & 2.24 & -3.16 & -5.40 & 1 & 1 & 0.05 & \\
\hline 27 & 2.23 & -3.16 & -5.40 & 1 & & & \\
\hline 28 & 2.23 & -3.16 & -5.40 & 1 & & & \\
\hline 29 & 2.23 & -3.17 & -5.40 & 1 & & & \\
\hline 30 & 2.23 & -3.17 & -5.40 & 1 & & & \\
\hline 31 & 2.23 & -3.17 & -5.40 & 1 & & & \\
\hline 32 & 2.23 & -3.17 & -5.40 & 1 & & & \\
\hline 33 & 2.23 & -3.17 & -5.40 & 1 & & & \\
\hline 34 & 2.23 & -3.17 & -5.40 & 2 & & & \\
\hline 35 & 2.23 & -3.17 & -5.40 & 2 & & & \\
\hline 36 & 2.23 & -3.17 & -5.40 & 2 & & & \\
\hline 37 & 2.23 & -3.17 & -5.40 & 2 & & & \\
\hline 38 & 2.22 & -3.17 & -5.40 & 2 & & & \\
\hline 39 & 2.22 & -3.17 & -5.40 & 2 & & & \\
\hline 40 & 2.22 & -3.17 & -5.40 & 1 & 1 & 0.04 & \\
\hline 41 & 2.22 & -3.18 & -5.40 & 1 & & & \\
\hline 42 & 2.22 & -3.18 & -5.40 & 1 & & & \\
\hline 43 & 2.22 & -3.18 & -5.40 & 1 & & & \\
\hline 44 & 2.22 & -3.18 & -5.40 & 1 & & & \\
\hline 45 & 2.22 & -3.18 & -5.40 & 2 & & & \\
\hline 46 & 2.22 & -3.18 & -5.40 & 2 & & & \\
\hline 47 & 2.22 & -3.18 & -5.40 & 2 & & & \\
\hline 48 & 2.22 & -3.18 & -5.40 & 2 & & & \\
\hline 49 & 2.22 & -3.18 & -5.40 & 2 & & & \\
\hline 50 & 2.21 & -3.18 & -5.40 & 2 & & & \\
\hline
\end{tabular}

EVOUVE: SUCCESS. TIME EVOLUTION COMPLETED. 
Species Name Mole_frac Activity Density $\left(\mathrm{gm} / \mathrm{cm}^{\star \star} 3\right)$

$$
\text { mole }
$$

$\mathrm{N}(\mathrm{D})=1.000^{\circ} \quad 1.000 \quad 1.37$

$\begin{array}{lcl}\text { RES IDENCE TIME } & 0.2870 \mathrm{E}-02 & \text { SEC } \\ \text { MASS FIOW RAIE } & 0.2268 \mathrm{E}+00 & \text { GM/SEC } \\ \text { PRESSURE } & 0.2368 \mathrm{E}-02 & \text { ATM } \\ \text { MASS DENSITY } & 0.3255 \mathrm{E}-06 & \mathrm{GM} / \mathrm{CMB} \\ \text { VOLUME } & 2000 . & \mathrm{CMB} \\ \text { SURFACE AREA } & 950.0 & \mathrm{CM} \\ \text { SURFACE TO VULUME RATIO } & 0.4750 & \mathrm{CM}-1 \\ \text { TEMPERATURE (FIXED) } & 1440 . & \mathrm{K}\end{array}$


PRINTOUT OF RAW RESIDIALS:

$\begin{array}{llc}\text { Temperature } & : & 0.000 \mathrm{E}+00 \\ \mathrm{H} 2 & : & -28.6 \\ \mathrm{H} & : & -0.480 \mathrm{E}-02 \\ \mathrm{~N} 2 & : & -133 . \\ \mathrm{N} & : & 0.151 \mathrm{E}-05 \\ \mathrm{NH} & : & 0.109 \mathrm{E}-03 \\ \mathrm{NH} 2 & : & 0.297 \mathrm{E}-01 \\ \mathrm{NNH} & : & 0.415 \mathrm{E}-09 \\ \mathrm{~N} 2 \mathrm{H} 2 & : & 0.162 \mathrm{E}-06 \\ \mathrm{~N} 2 \mathrm{H} 3 & : & 0.705 \mathrm{E}-08 \\ \mathrm{~N} 2 \mathrm{H} 4 & : & 0.114 \mathrm{E}-08 \\ \mathrm{HF} & : & -0.436 \\ \text { F } & : & -0.733 \mathrm{E}-07 \\ \text { SIF4 } & : & 9.42 \\ \text { SIF3 } & : & -0.287 \mathrm{E}-01 \\ \text { SIHF3 } & : & -10.7 \\ \text { SIF3NH2 } & : & -0.944 \mathrm{E}-03 \\ \text { NH3 } & : & 164 .\end{array}$

Surface phase site fraction residuals:

$\begin{array}{llc}\text { HN_SIF (S) } & : & 2.70 \\ \text { F3SI_NHL (S) } & : & 0.266 \mathrm{E}-0 \\ \text { F2SINH (S) } & : & 15.5 \\ \text { H2NFSINH (S) } & : & -0.601 \\ \text { HN } \text { FSINH)2(S) } & : & -0.529 \\ \text { HN_NH2 (S) } & : & -17.1\end{array}$

Bulk phase mole fraction residuals:

$S I(D) \quad: \quad 0.000 E+00$

$\mathrm{N}(\mathrm{D}) \quad: \quad 0.000 \mathrm{E}+00$

TWOENT: EVOLVE PERFORMED A TTME EVOUTION.

TWOANT: CALLING SEARCH TO SOLVE THE STEADY STATE PROEIEM.

SEARCH: SOLVE NONIINEAR, NONDIFFERENTIAL EQATIIONS.

\begin{tabular}{|c|c|c|c|c|c|c|c|}
\hline \multirow{2}{*}{$\begin{array}{r}\text { SITN } \\
\text { NLMBER }\end{array}$} & \multicolumn{7}{|l|}{ LOG10 } \\
\hline & NORM F & COND J & NORM S & ABS & AND REL & DEITA & $A N D D$ \\
\hline 0 & 2.21 & 3.04 & -0.09 & -0.09 & 3.96 & -1.83 & \\
\hline 1 & 2.21 & 2.84 & -0.16 & -0.16 & 2.55 & & -0.30 \\
\hline 2 & 1.95 & & -0.19 & -0.19 & 1.11 & -1.10 & \\
\hline 3 & 1.92 & & -0.28 & -0.28 & 2.18 & -0.25 & -0.30 \\
\hline 4 & 1.82 & & -0.88 & -0.88 & 1.77 & -0.70 & \\
\hline 5 & 1.76 & & -0.94 & -0.94 & 2.17 & & -0.60 \\
\hline 6 & 1.68 & & -0.98 & -0.98 & 2.47 & -0.61 & -0.30 \\
\hline 7 & 1.64 & & -1.01 & -1.01 & 1.14 & & -0.90 \\
\hline 8 & 1.61 & & -1.09 & -1.09 & 1.22 & -0.29 & -0.60 \\
\hline 9 & 1.57 & & -1.09 & -1.09 & 0.70 & & -0.90 \\
\hline 10 & 1.54 & & -1.15 & -1.15 & 1.07 & -0.32 & -0.90 \\
\hline 11 & 1.53 & & -1.17 & -1.17 & 0.45 & & \\
\hline 12 & 1.20 & & -1.43 & -1.43 & 0.88 & & -0.90 \\
\hline 13 & 1.17 & & -1.52 & -1.52 & 2.43 & & -0.60 \\
\hline 14 & 1.12 & & -1.58 & -1.58 & 1.40 & & -1.20 \\
\hline 15 & 1.10 & & -1.59 & -1.59 & 1.71 & & -0.90 \\
\hline 16 & 1.08 & & -1.61 & -1.61 & 1.24 & & -1.20 \\
\hline 17 & 1.06 & & -1.63 & -1.63 & 1.93 & & -0.90 \\
\hline 18 & 1.04 & & -1.66 & -1.66 & 1.22 & & -1.20 \\
\hline 19 & 1.03 & & -1.67 & -1.67 & 2.29 & & -0.90 \\
\hline 20 & 1.00 & & -1.68 & -1.68 & 1.21 & & -1.20 \\
\hline
\end{tabular}




$\begin{array}{rrrrrr}21 & 0.99 & 1.99 & -1.36 & -1.36 & 1.28 \\ 22 & -0.22 & & -2.58 & -2.58 & 0.05 \\ 23 & -1.14 & & -3.49 & -3.49 & -0.04 \\ 24 & -1.84 & & -4.39 & -4.39 & 0.01 \\ 25 & -2.87 & & -5.28 & -5.28 & 0.27 \\ 26 & -3.41 & & -6.18 & -6.18 & -0.06 \\ 27 & -4.45 & & -7.03 & -7.03 & -0.07 \\ 28 & -4.89 & & -7.78 & -7.78 & -0.09 \\ 29 & -5.21 & & -8.30 & -8.30 & 0.03 \\ 30 & -5.46 & & -8.56 & -8.56 & 0.88 \\ 31 & -5.73 & & -8.82 & -8.82 & -0.22 \\ 32 & -5.99 & & -9.09 & -9.09 & -0.16 \\ 33 & -6.27 & & -9.36 & -9.36 & -0.01 \\ 34 & -6.55 & & -9.64 & -9.64 & 1.16 \\ 35 & -6.83 & & -9.94 & -9.94 & -0.11 \\ 36 & -7.13 & & -10.25 & -10.25 & 0.15 \\ 37 & -7.45 & & -10.59 & -10.59 & 0.35 \\ 38 & -7.79 & & -10.91 & -10.97 & 0.37 \\ 39 & -8.18 & & -11.27 & -11.41 & 0.55 \\ 40 & -8.65 & & -11.53 & -11.53 & -0.33 \\ 41 & -8.81 & 2.01 & -11.19 & -11.19 & 0.04 \\ 42 & -13.40 & & -15.68 & -17.19 & -0.32 \\ 43 & -14.20 & & -16.88 & -25.00 & -15.64\end{array}$

SEARCH: SUCCESS. THE SOUUTION: PSPRNI: Printing of arrent solution from TWORNT:

$\begin{array}{lcl}\text { RESIDENCE TIME } & 0.5082 \mathrm{E}-02 & \mathrm{SEC} \\ \text { MASS FICN RATE } & 0.2268 \mathrm{E}+00 & \mathrm{GM} / \mathrm{SEC} \\ \text { PRESSURE } & 0.2368 \mathrm{E}-02 & \text { ATM } \\ \text { MASS DENSITY } & 0.5764 \mathrm{E}-06 & \mathrm{GM} / \mathrm{CMB} \\ \text { VOLUME } & 2000 . & \mathrm{CMB} \\ \text { SURFACE AREA } & 950.0 & \mathrm{CMR} \\ \text { SUREACE TO VOLUME RATIO } & 0.4750 & \mathrm{CM}-1 \\ \text { TEMPERATURE (FIXED) } & 1440 . & \mathrm{K}\end{array}$

EXIT GAS FHASE MOIE FRACTIONS

$\begin{array}{lllllll}\mathrm{H} 2 & =0.2109 \mathrm{E}-07 & \mathrm{H} & =0.3802 \mathrm{E}-09 & \mathrm{~N} 2 & =0.1090 \mathrm{E}-13 \\ \mathrm{~N} & =0.1932 \mathrm{E}-18 & \mathrm{NH} & =0.4932 \mathrm{E}-13 & \mathrm{NH} 2 & =0.4256 \mathrm{E}-07 \\ \mathrm{NNH} & =0.2120 \mathrm{E}-15 & \mathrm{~N} 2 \mathrm{H} 2 & =0.8357 \mathrm{E}-13 & \mathrm{~N} 2 \mathrm{H} 3 & =0.3595 \mathrm{E}-14 \\ \text { NRH } & =0.4790 \mathrm{E}-15 & \mathrm{HF} & =0.2941 \mathrm{E}-01 & \mathrm{~F} & =0.1310 \mathrm{E}-13 \\ \text { SIF4 } & =0.1338 & \mathrm{SIF3} & =0.3496 \mathrm{E}-13 & \text { SIHF3 } & =0.9155 \mathrm{E}-14 \\ \text { SIF3NH2 } & =0.8831 \mathrm{E}-13 & \mathrm{NHB} & & =0.8368 & & \end{array}$

SURFACE SITE FRACTIONS IN SURFACE PHASE, ST3NA

Site density $=0.4168 \mathrm{E}-08 \mathrm{~mole} / \mathrm{cm}^{\star *} 2$

Standard state Site density $=0.4168 \mathrm{E}-08 \mathrm{~mole} / \mathrm{cm}^{\star} \star 2$

Rate of change of site density $=0.0000 \mathrm{E}+00 \mathrm{~mole} /\left(\mathrm{cm}^{\star \star} 2^{\star} \mathrm{sec}\right)$

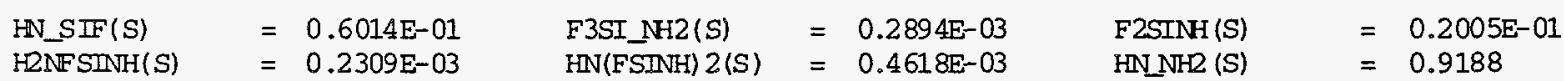

BUR FHASE MOLE FRACTIONS AND ACTIVITIES IN BUIK PHASE, BUKL

Total growth rate of bulk phase $=0.8200 \mathrm{E}-06 \mathrm{~cm} / \mathrm{sec}$ $=0.1609 \mathrm{E}-02 \mathrm{gm} / \mathrm{sec}$

Density of the bulk phase $=2.066 \mathrm{gm} / \mathrm{cm}^{\star * 3}$ Average molecular weight of bulk phase $=28.09 \mathrm{gm} / \mathrm{mole}$ 


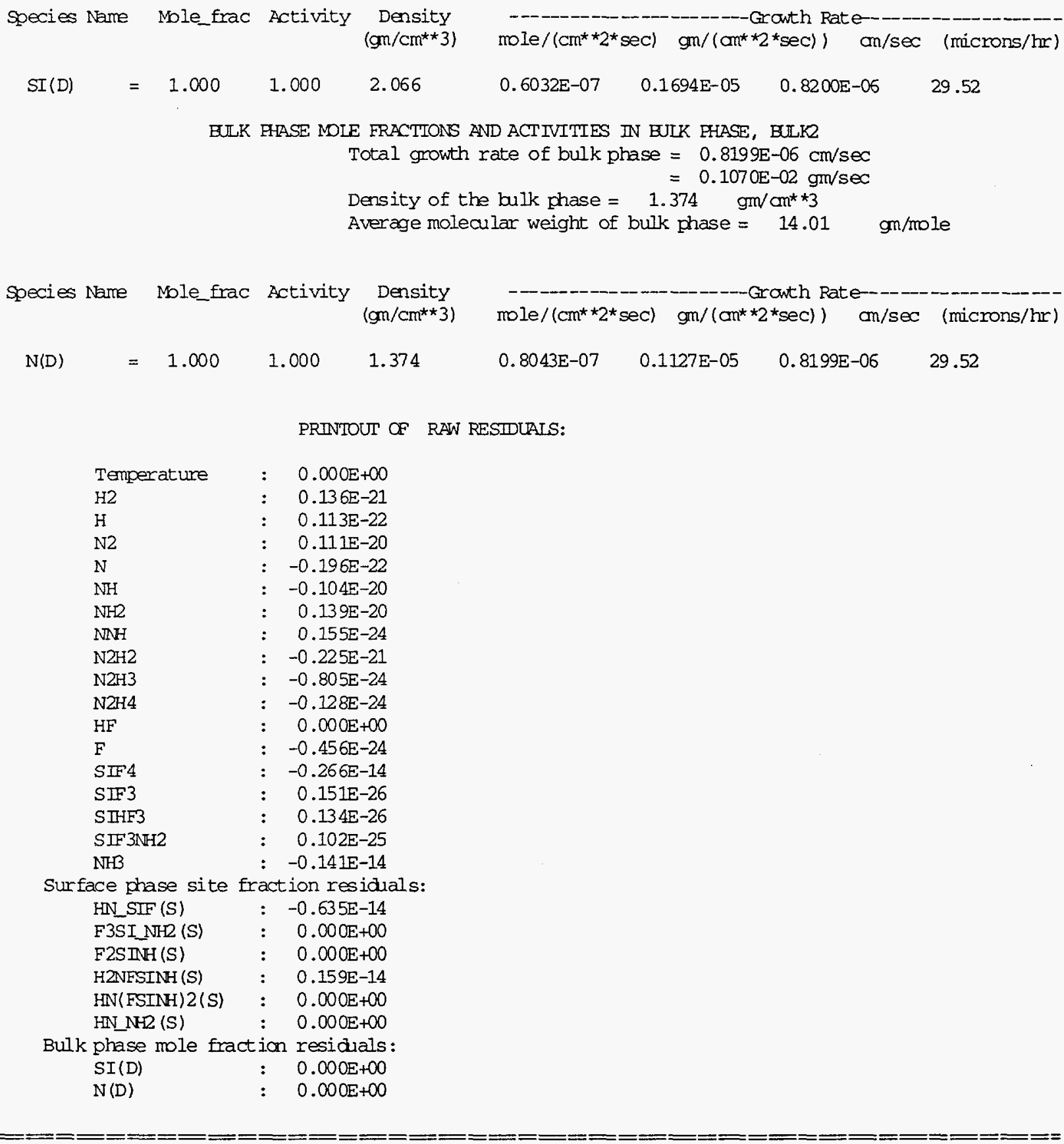

TWOENT: SEARCH FOUND THE STEADY STATE.

TWOANT: 0.76 SECONDS TOTAL COMPUTER TIME (SEE BREAKDONN BELOW).

SUBIASK

EVAT $F$ PREP J SOTVE OTHER

\% OF TOIAL MEAN SECONDS QUANTITY
27.6

0.001

270
43.4

0.027

12
6.6

0.000

219
TASK

EVOTVE SEARCH

22.4

46.1

51.3 
TWOENT: SUCCESS. PROBLEM SOLVED.

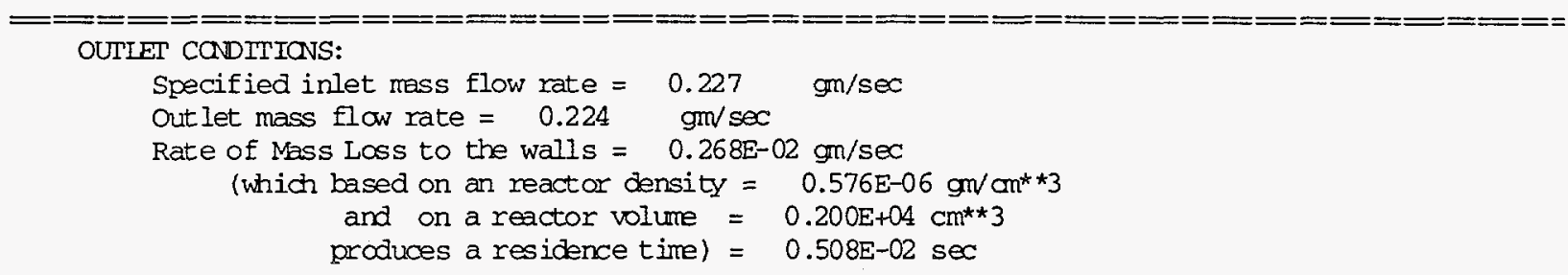

Outlet and reactor temperature $=1440.0 \quad$ Kelvin

Outlet and reactor pressure $=0.237 \mathrm{E}-02 \mathrm{~atm}$

Out let and reactor density $=0.57638 \mathrm{E}-06 \mathrm{gm} / \mathrm{cm}^{\star *} 3$

Outlet and reactor mean molecular weight $=28.761$

Outlet molax flow rate $\quad=0.77934 \mathrm{E}-02 \mathrm{moles} / \mathrm{sec}$

$\mathrm{gn} / \mathrm{mole}$

Out let volumetric flow rate $=0.38889 \mathrm{E}+06 \mathrm{~cm}^{\star *} 3 / \mathrm{sec}$

(based on reactor pressure and temperature)

$$
\begin{array}{ll}
=11440 . & \text { SCCM } \\
=11.440 & \text { SIPM }
\end{array}
$$

OUTLET CONDITIONS FCR GAS PHASE MOIECULAR SPECIES:

\begin{tabular}{lcccccc} 
Species & molefrac & $\# / \mathrm{cn} 3$ & $\mathrm{moles} / \mathrm{sec}$ & $\mathrm{gm} / \mathrm{sec}$ & $\mathrm{cm} \star * 3 / \mathrm{sec}$ & SCCM \\
\hline H2 & $0.21088 \mathrm{E}-07$ & $0.25449 \mathrm{E}+09$ & $0.16435 \mathrm{E}-09$ & $0.33132 \mathrm{E}-09$ & $0.82010 \mathrm{E}-02$ & $0.24125 \mathrm{E}-03$ \\
H & $0.38017 \mathrm{E}-09$ & $0.45879 \mathrm{E}+07$ & $0.29628 \mathrm{E}-11$ & $0.29864 \mathrm{E}-11$ & $0.14784 \mathrm{E}-03$ & $0.43492 \mathrm{E}-05$ \\
NR & $0.10903 \mathrm{E}-13$ & 131.58 & $0.84970 \mathrm{E}-16$ & $0.23803 \mathrm{E}-14$ & $0.42400 \mathrm{E}-08$ & $0.12473 \mathrm{E}-09$ \\
N & $0.19319 \mathrm{E}-18$ & $0.23315 \mathrm{E}-02$ & $0.15056 \mathrm{E}-20$ & $0.21089 \mathrm{E}-19$ & $0.75131 \mathrm{E}-13$ & $0.22102 \mathrm{E}-14$ \\
NH & $0.49324 \mathrm{E}-13$ & 595.24 & $0.38440 \mathrm{E}-15$ & $0.57716 \mathrm{E}-14$ & $0.19182 \mathrm{E}-07$ & $0.56427 \mathrm{E}-09$ \\
NH2 & $0.42556 \mathrm{E}-07$ & $0.51357 \mathrm{E}+09$ & $0.33166 \mathrm{E}-09$ & $0.53140 \mathrm{E}-08$ & $0.16550 \mathrm{E}-01$ & $0.48685 \mathrm{E}-03$ \\
NNH & $0.21200 \mathrm{E}-15$ & 2.5584 & $0.16522 \mathrm{E}-17$ & $0.47949 \mathrm{E}-16$ & $0.82445 \mathrm{E}-10$ & $0.24253 \mathrm{E}-11$ \\
NRH2 & $0.83573 \mathrm{E}-13$ & 1008.6 & $0.65132 \mathrm{E}-15$ & $0.19559 \mathrm{E}-13$ & $0.32501 \mathrm{E}-07$ & $0.95610 \mathrm{E}-09$ \\
NRH3 & $0.35955 \mathrm{E}-14$ & 43.391 & $0.28021 \mathrm{E}-16$ & $0.86970 \mathrm{E}-15$ & $0.13983 \mathrm{E}-08$ & $0.41133 \mathrm{E}-10$ \\
NRH4 & $0.47900 \mathrm{E}-15$ & 5.7806 & $0.37331 \mathrm{E}-17$ & $0.11963 \mathrm{E}-15$ & $0.18628 \mathrm{E}-09$ & $0.54799 \mathrm{E}-11$ \\
HF & $0.29411 \mathrm{E}-01$ & $0.35494 \mathrm{E}+15$ & $0.22921 \mathrm{E}-03$ & $0.45857 \mathrm{E}-02$ & 11438. & 336.47 \\
F & $0.13099 \mathrm{E}-13$ & 158.08 & $0.10208 \mathrm{E}-15$ & $0.19394 \mathrm{E}-14$ & $0.50940 \mathrm{E}-08$ & $0.14985 \mathrm{E}-09$ \\
SIF4 & 0.13376 & $0.16142 \mathrm{E}+16$ & $0.10424 \mathrm{E}-02$ & 0.10849 & 52017. & 1530.2 \\
SIF3 & $0.34960 \mathrm{E}-13$ & 421.91 & $0.27246 \mathrm{E}-15$ & $0.23181 \mathrm{E}-13$ & $0.13596 \mathrm{E}-07$ & $0.39995 \mathrm{E}-09$ \\
SIHF3 & $0.91548 \mathrm{E}-14$ & 110.48 & $0.71347 \mathrm{E}-16$ & $0.61422 \mathrm{E}-14$ & $0.35602 \mathrm{E}-08$ & $0.10473 \mathrm{E}-09$ \\
SIF3N2 & $0.88309 \mathrm{E}-13$ & 1065.7 & $0.68823 \mathrm{E}-15$ & $0.69583 \mathrm{E}-13$ & $0.34343 \mathrm{E}-07$ & $0.10103 \mathrm{E}-08$ \\
NH3 & 0.83683 & $0.10099 \mathrm{E}+17$ & $0.65218 \mathrm{E}-02$ & 0.11107 & $0.32544 \mathrm{E}+06$ & 9573.5
\end{tabular}

DEIAIIED SPECIES BALANCE

(all rates are in moles per sec)

\begin{tabular}{lcccccc}
\multicolumn{1}{c}{ SPECIES } & INLET_FR & OUILET_FR & GAS_PROD_RATE & GAS_DEST_RATE & SURE_NET_PRCD & TOTAL_NET \\
\hline H2 & $0.000 \mathrm{E}+00$ & $1.643 \mathrm{E}-10$ & $1.643 \mathrm{E}-10$ & $2.217 \mathrm{E}-16$ & $0.000 \mathrm{E}+00$ & $5.170 \mathrm{E}-26$ \\
$\mathrm{H}$ & $0.000 \mathrm{E}+00$ & $2.963 \mathrm{E}-12$ & $1.673 \mathrm{E}-10$ & $1.643 \mathrm{E}-10$ & $0.000 \mathrm{E}+00$ & $2.585 \mathrm{E}-26$ \\
$\mathrm{NR}$ & $0.000 \mathrm{E}+00$ & $8.497 \mathrm{E}-17$ & $8.497 \mathrm{E}-17$ & $1.090 \mathrm{E}-28$ & $0.000 \mathrm{E}+00$ & $4.584 \mathrm{E}-26$ \\
$\mathrm{~N}$ & $0.000 \mathrm{E}+00$ & $1.506 \mathrm{E}-21$ & $1.506 \mathrm{E}-21$ & $4.757 \mathrm{E}-25$ & $0.000 \mathrm{E}+00$ & $-1.610 \mathrm{E}-27$ \\
$\mathrm{NH}$ & $0.000 \mathrm{E}+00$ & $3.844 \mathrm{E}-16$ & $4.732 \mathrm{E}-16$ & $8.880 \mathrm{E}-17$ & $0.000 \mathrm{E}+00$ & $-7.954 \mathrm{E}-26$ \\
$\mathrm{NH2}$ & $0.000 \mathrm{E}+00$ & $3.317 \mathrm{E}-10$ & $3.318 \mathrm{E}-10$ & $1.097 \mathrm{E}-13$ & $0.000 \mathrm{E}+00$ & $1.034 \mathrm{E}-25$ \\
$\mathrm{NNH}$ & $0.000 \mathrm{E}+00$ & $1.652 \mathrm{E}-18$ & $8.662 \mathrm{E}-17$ & $8.497 \mathrm{E}-17$ & $0.000 \mathrm{E}+00$ & $6.163 \mathrm{E}-30$ \\
$\mathrm{NRH2}$ & $0.000 \mathrm{E}+00$ & $6.513 \mathrm{E}-16$ & $7.379 \mathrm{E}-16$ & $8.662 \mathrm{E}-17$ & $0.000 \mathrm{E}+00$ & $-8.643 \mathrm{E}-27$ \\
$\mathrm{~N} 2 \mathrm{H} 3$ & $0.000 \mathrm{E}+00$ & $2.802 \mathrm{E}-17$ & $3.859 \mathrm{E}-17$ & $1.057 \mathrm{E}-17$ & $0.000 \mathrm{E}+00$ & $-2.990 \mathrm{E}-29$
\end{tabular}




$\begin{array}{lllllrr}\text { N2H4 } & 0.000 \mathrm{E}+00 & 3.733 \mathrm{E}-18 & 6.087 \mathrm{E}-18 & 2.354 \mathrm{E}-18 & 0.000 \mathrm{E}+00 & -4.602 \mathrm{E}-30 \\ \text { HF } & 0.000 \mathrm{E}+00 & 2.292 \mathrm{E}-04 & 1.079 \mathrm{E}-13 & 1.069 \mathrm{E}-13 & 2.292 \mathrm{E}-04 & -5.421 \mathrm{E}-20 \\ \text { F } & 0.000 \mathrm{E}+00 & 1.021 \mathrm{E}-16 & 1.080 \mathrm{E}-13 & 1.079 \mathrm{E}-13 & 0.000 \mathrm{E}+00 & -2.524 \mathrm{E}-29 \\ \text { SIF4 } & 1.100 \mathrm{E}-03 & 1.042 \mathrm{E}-03 & 2.208 \mathrm{E}-20 & 1.032 \mathrm{E}-15 & -5.730 \mathrm{E}-05 & -2.168 \mathrm{E}-19 \\ \text { SIF3 } & 0.000 \mathrm{E}+00 & 2.725 \mathrm{E}-16 & 7.533 \mathrm{E}-16 & 4.808 \mathrm{E}-16 & 0.000 \mathrm{E}+00 & -9.861 \mathrm{E}-32 \\ \text { SIHF3 } & 0.000 \mathrm{E}+00 & 7.135 \mathrm{E}-17 & 7.135 \mathrm{E}-17 & 5.834 \mathrm{E}-24 & 0.000 \mathrm{E}+00 & 2.465 \mathrm{E}-32 \\ \text { SIF3NH2 } & 0.000 \mathrm{E}+00 & 6.882 \mathrm{E}-16 & 6.882 \mathrm{E}-16 & 3.160 \mathrm{E}-27 & 0.000 \mathrm{E}+00 & 0.000 \mathrm{E}+00 \\ \text { NH3 } & 6.598 \mathrm{E}-03 & 6.522 \mathrm{E}-03 & 1.074 \mathrm{E}-13 & 3.318 \mathrm{E}-10 & -7.640 \mathrm{E}-05 & -8.674 \mathrm{E}-19 \\ \text { HN_SIF(S) } & 0.000 \mathrm{E}+00 & 0.000 \mathrm{E}+00 & 0.000 \mathrm{E}+00 & 0.000 \mathrm{E}+00 & -1.257 \mathrm{E}-20 & -1.257 \mathrm{E}-20 \\ \text { F3SI_NH2(S) } & 0.000 \mathrm{E}+00 & 0.000 \mathrm{E}+00 & 0.000 \mathrm{E}+00 & 0.000 \mathrm{E}+00 & 0.000 \mathrm{E}+00 & 0.000 \mathrm{E}+00 \\ \text { F2SINH(S) } & 0.000 \mathrm{E}+00 & 0.000 \mathrm{E}+00 & 0.000 \mathrm{E}+00 & 0.000 \mathrm{E}+00 & 0.000 \mathrm{E}+00 & 0.000 \mathrm{E}+00 \\ \text { H2NESINH(S) } & 0.000 \mathrm{E}+00 & 0.000 \mathrm{E}+00 & 0.000 \mathrm{E}+00 & 0.000 \mathrm{E}+00 & 3.143 \mathrm{E}-21 & 3.143 \mathrm{E}-21 \\ \text { HN (ESINH) 2(S) } & 0.000 \mathrm{E}+00 & 0.000 \mathrm{E}+00 & 0.000 \mathrm{E}+00 & 0.000 \mathrm{E}+00 & 0.000 \mathrm{E}+00 & 0.000 \mathrm{E}+00 \\ \text { HN_NH2(S) } & 0.000 \mathrm{E}+00 & 0.000 \mathrm{E}+00 & 0.000 \mathrm{E}+00 & 0.000 \mathrm{E}+00 & 0.000 \mathrm{E}+00 & 0.000 \mathrm{E}+00 \\ \text { SI (D) } & 0.000 \mathrm{E}+00 & 0.000 \mathrm{E}+00 & 0.000 \mathrm{E}+00 & 0.000 \mathrm{E}+00 & 5.730 \mathrm{E}-05 & 5.730 \mathrm{E}-05 \\ \text { N(D) } & 0.000 \mathrm{E}+00 & 0.000 \mathrm{E}+00 & 0.000 \mathrm{E}+00 & 0.000 \mathrm{E}+00 & 7.640 \mathrm{E}-05 & 7.640 \mathrm{E}-05\end{array}$

\section{DEIATLED ELEMENT BALANCES}

(all rates are in moles per sec)

\begin{tabular}{|c|c|c|c|c|c|}
\hline ELEMENT & INLET_FR & OTILET_FR & EULK1 & BULK2 & TOTAL_NET \\
\hline $\mathrm{H}$ & $1.979 \mathrm{E}-02$ & $1.979 E-02$ & $0.000 E+00$ & $0.000 E+00$ & $0.000 E+00$ \\
\hline $\mathrm{N}$ & $6.598 \mathrm{E}-03$ & $6.522 \mathrm{E}-03$ & $0.000 E+00$ & $7.640 \mathrm{E}-05$ & $-3.253 E-19$ \\
\hline SI & $1.100 \mathrm{E}-03$ & 1. $042 \mathrm{E}-03$ & $5.730 E-05$ & $0.000 E+00$ & $-2.507 \mathrm{E}-19$ \\
\hline$F$ & $4.399 \mathrm{E}-03$ & $4.399 E-03$ & $0.000 E+00$ & $0.000 E+00$ & $0.000 E+00$ \\
\hline
\end{tabular}

LOGARITHMIC SENSITIVITY COEFFICIENIS FOR GAS PHASE REACTIONS

Threshold normalized value for printing of SEN coefficients $=0.10000 \mathrm{E}-01$

Threshold nomblized value for printing of temperature sensitivity coefficient $=0.10000 \mathrm{E}-03$ Threshold normalized value for printing of growth rate sensitivity coefficient $=0.10000 \mathrm{E}-03$

LOCARITHMTC SENSITIVITY COEFFICIENTS FOR SURFACE FHASE REACIIONS:

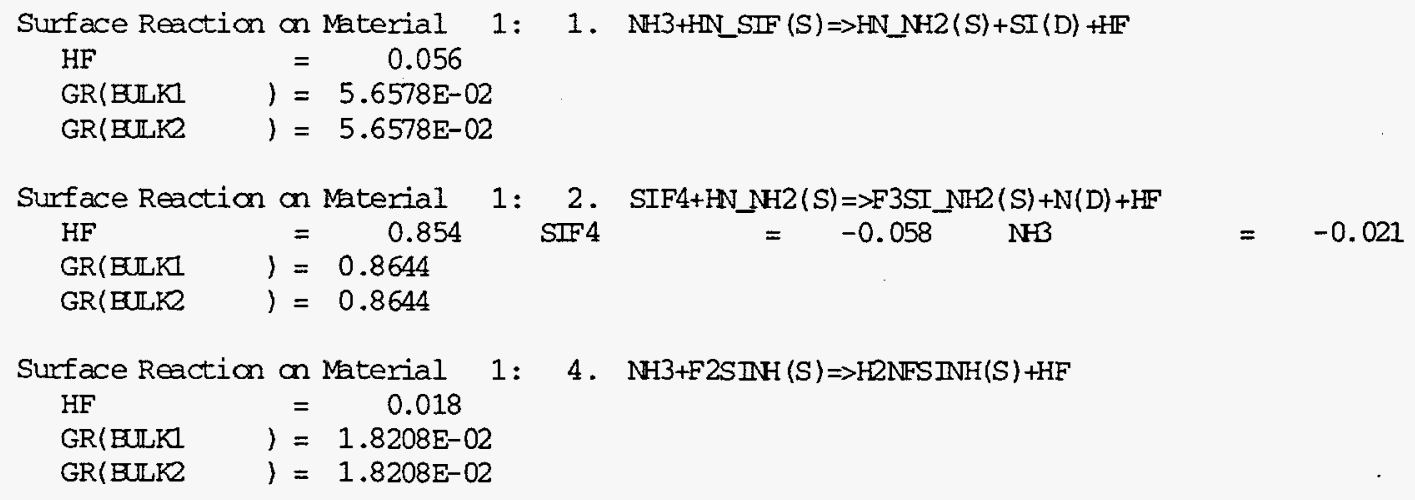

SENSITIVITY CALCULATION COMPIETE 


\section{SAMPLE PROBLEM B: LOW-PRESSURE $\mathrm{Cl}_{2}$ PLASMA}

The next example problem we present is a low-pressure, chlorine plasma reactor. The chemistry mechanisms now include electrons and positive and negative ions, as well as neutral species. Note that the electron must appear in the list of ELEMENTS in the CHEMKIN Input file. Also, any element that is introduced in the SURFACE CHEMKIN mechanism must appear in the CHEMKIN ELEMENTS list. The thermodynamic data includes each species elemental composition. More information about the thermodynamic data and other CHEMKIN input is available in the CHEMKIN-III User's Manual. ${ }^{2}$ The reactions listed in the CHEMKIN Input file include electron-impact ionization, dissociation, dissociative attachment, and excitation reactions. The fact that these reactions depend on the electron temperature (not the gas temperature) is indicated by the auxiliary keyword, "TDEP," with the electron indicated by the "E" between the slashes that follow "TDEP." Reactions where the user wishes to specify explicitly the energy loss per collision, use the keyword "EXCI," followed by the energy loss in units of $\mathrm{eV}$. This option is used to describe excitation energy losses for electrons, without requiring the user to define each excited state as a separate species with corresponding thermodynamic data. The use of the "EXCI" keyword for multiple excitation reactions often requires the additional keyword "DUP." Ordinarily CHEMKIN will flag multiple reaction strings that look identical as errors, unless the "DUP" keyword is included. These keywords are described fully in the CHEMKIN-III manual. ${ }^{2}$

The SURFACE CHEMKIN mechanism shown here for the chlorine plasma system is relatively simple. No etch reactions have been included in this example. However the surface reactions that are listed typically play a very crucial role in determining the plasma composition. ${ }^{21,20}$ First, we want to consider adsorption and abstraction of atomic chlorine on the surfaces in the reactor chamber. For this reason we introduce both open and chlorinated surface sites. The overall surface site density is assigned the value of $2.5 \times 10^{-9}$ moles $/ \mathrm{cm}^{2}$. Thermodynamic data must be provided for the surface species. However, since we specify all the reactions as irreversible, this thermodynamic data is not actually used in determining the reaction rates. For this reason, we have somewhat arbitrarily defined the composition of the surface to be silicon and used "dummy" values for the thermodynamic fits. On the reaction statement, the word, MWOFF, is included. This indicates that we do not wish to use the MotzWise correction factor for large sticking coefficients. ${ }^{40}$ Instead, the sticking coefficient is defined simply as the reaction rate divided by the incoming flux of the species. The first two reactions listed describe the ions recombining with electrons at the surface. These reactions have been flagged as BOHM reactions. This indicates that the ion flux is limited by the Bohm velocity of the ions, rather than by the ion's thermal speed. The implementation of the Bohm condition is described in detail in Section II. The reaction rate coefficients for this reaction are listed as '0.4 $0.0 \quad 0.0^{\prime}$, which indicates that the Bohm flux should be multiplied by a factor ' 0.4 ', similar to the way a sticking coefficient is used. This factor has been introduced here to account for the fact that the ion interaction with the 
surface will be subject to transport limitations, such that a gradient near the walls in the ion density will occur. Since the AURORA model cannot capture this effect, the correction to the Bohm flux is introduced in the SURFACE CHEMKIN mechanism. This factor represents the ratio between the ion density at the sheath edge and the ion density in the bulk of the plasma. An alternative approach to assigning a value of less than unity to the Bohm coefficient in the SURFACE CHEMKIN file, is to use the BOHM keyword in the AURORA input file. This keyword would cause AURORA to adjust the total ion flux for each ion to be the Bohm flux multiplied by the value specified in the AURORA BOHM input. The next two reactions in the surface mechanism represent the interaction between atomic chlorine and the reactor walls, causing a net recombination of atomic chlorine to form molecular chlorine. The last reaction represents the de-excitation of the metastable species $\mathrm{Cl}^{*}$ at reactor surfaces. These reactions have been specified using sticking coefficients.

The output file from AURORA begins with an echoing of the input keywords, including any comment lines (preceded by a "!"). Here we have included comment lines that describe each keyword within the input file. These comments regarding the keyword definitions facilitate editing and changing the input file for new problems. The input keywords listed include ENGE, which indicates we are solving the electron energy equation. This requires the initial guess for the electron temperature to be input using ETMP, as well as an inlet electron temperature "TEIN." The inlet electron temperature actually has no impact on the solution unless there are electrons specified in the REAC keywords. The keyword NOFT indicates that we wish to skip the fixed-temperature solution. This will cause AURORA to attempt to solve simultaneously all equations the first time through. For plasma simulations this is usually more robust than trying to solve the fixed-temperature solution first. TION is the ion temperature in Kelvin. The power deposited into the plasma is indicated by PWRW and is given in Watts. The parameter ELSH describes the ion energy gained crossing the sheath and may be specified differently for each material in the system. For this case, we only have one material, and the default name of unnamed materials in SURFACE CHEMKIN are MATERIAL1, MATERIAL2, etc. Note that we have included both BOHM in the AURORA keyword input, as well as using a less-thanunity BOHM coefficient in the SURFACE CHEMKIN file. Strictly speaking, this is redundant, but the net effect will be the same as using one or the other approach alone. Other information needed for the electron energy equation includes the specification of electron-neutral elastic collision cross sections using XSEK or XSDF. The specification of a reactor heat loss through QLOS or HTRN is necessary to solve the gas energy equation. PRMT is the pressure of the reactor in units of millitorr. Since we only have one material in the surface mechanism we need not specify area fractions using the keyword AFRA. However, two commented lines have been included to demonstrate how these keywords would be used in the case that two materials were specified having the names WALL and WAFER. The remainder of the keywords are similar to the thermal CVD example given in the previous Section.

The output file has less information about the TWOPNT procedure printed than in the thermal CVD example of Section VI, because we have used the default PRNT value of 1 . Some new information is printed for the plasma simulations. In the initial guess, the electron temperature estimate is shown, 
and the residual for the electron temperature is also printed. For the final solution, the electron temperature is printed in both Kelvin and electron Volt units. Also printed is the charge imbalance of the solution, calculated as the difference between the sum of positive ion densities minus the sum of the electron density and the negative ion densities, divided by the total positive ion density. This is a useful diagnostic to determine whether or not TWOPNT has arrived at a physically reasonable solution. This percentage imbalance should be less than 1.0 , as a general rule. 


\section{CHEMKIN Input for the $\mathrm{Cl}_{2}$ Plasma Example}

EIEMENTS E CI SI END

SPECIES $\mathrm{E} C \mathrm{CL}+\mathrm{CL} 2+\mathrm{CL}-\mathrm{CL} * \mathrm{CL} \mathrm{CL} 2$

END

THERMD ALL

30010005000

$\begin{array}{lllllll}71091 \mathrm{E} & 1 & 0 & 0 & 0 \mathrm{G} & 300.000 & 10000.0001000 .00\end{array}$

$0.25002515 \mathrm{E}+01 \quad 0.00000000 \mathrm{E}+00 \quad 0.00000000 \mathrm{E}+00 \quad 0.00000000 \mathrm{E}+00 \quad 0.00000000 \mathrm{E}+00$

$-0.74597845 \mathrm{E}+03-0.11736856 \mathrm{E}+02 \quad 0.25002515 \mathrm{E}+01 \quad 0.00000000 \mathrm{E}+00 \quad 0.00000000 \mathrm{E}+00$

$0.00000000 \mathrm{E}+00 \quad 0.00000000 \mathrm{E}+00-0.74597839 \mathrm{E}+03-0.11736856 \mathrm{E}+02$

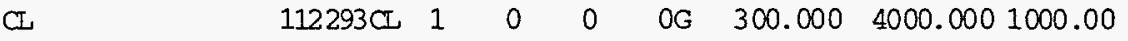

$0.29181864 \mathrm{E}+01-0.35438285 \mathrm{E}-03 \quad 0.12474453 \mathrm{E}-06-0.20015567 \mathrm{E}-100.11864538 \mathrm{E}-14$

$\begin{array}{lllll}0.13713931 E+05 & 0.32732935 \mathrm{E}+01 & 0.23711388 \mathrm{E}+01 & 0.96051756 \mathrm{E}-03 & 0.23850609 \mathrm{E}-06\end{array}$ $\begin{array}{llll}-0.20040531 E-08 & 0.11036082 \mathrm{E}-11 & 0.13841132 \mathrm{E}+05 & 0.60661221 \mathrm{E}+01\end{array}$

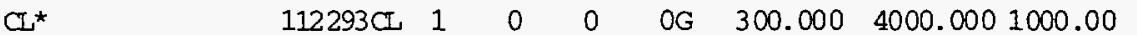

$0.29181864 \mathrm{E}+01-0.35438285 \mathrm{E}-03 \quad 0.12474453 \mathrm{E}-06-0.20015567 \mathrm{E}-10 \quad 0.11864538 \mathrm{E}-14$

$0.13713931 \mathrm{E}+05 \quad 0.32732935 \mathrm{E}+01 \quad 0.23711388 \mathrm{E}+01 \quad 0.96051756 \mathrm{E}-03 \quad 0.23850609 \mathrm{E}-06$

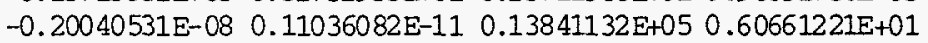

CIt $\quad 112293 \mathrm{CL}$ 1E

$0.30344462 \mathrm{E}+01-0.48197978 \mathrm{E}-03 \quad 0.15346743 \mathrm{E}-06-0.12604539 \mathrm{E}-10-0.32295198 \mathrm{E}-15$

$0.16550063 \mathrm{E}+06 \quad 0.29831080 \mathrm{E}+01 \quad 0.25186882 \mathrm{E}+01 \quad 0.99940156 \mathrm{E}-03 \quad 0.96029623 \mathrm{E}-07$

$\begin{array}{llll}-0.22788400 \mathrm{E}-08 & 0.13577272 \mathrm{E}-11 & 0.16558978 \mathrm{E}+06 & 0.55090742 \mathrm{E}+01\end{array}$

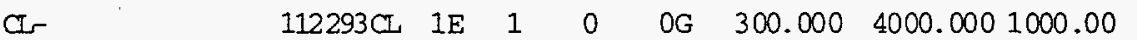

$0.25002515 \mathrm{E}+01 \quad 0.00000000 \mathrm{E}+00 \quad 0.00000000 \mathrm{E}+00 \quad 0.00000000 \mathrm{E}+00 \quad 0.00000000 \mathrm{E}+00$

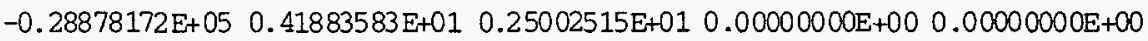

$0.00000000 \mathrm{E}+00 \quad 0.00000000 \mathrm{E}+00-0.28878168 \mathrm{E}+05 \quad 0.41883578 \mathrm{E}+01$

C2 $112293 \mathrm{CL} \quad 2 \quad 0 \quad 00$ OG $300.000 \quad 4000.0001000 .00$

$0.41252842 \mathrm{E}+01 \quad 0.68196526 \mathrm{E}-03-0.40838054 \mathrm{E}-06 \quad 0.11656516 \mathrm{E}-09-0.11435189 \mathrm{E}-13$

$\begin{array}{llll}-0.12794418 \mathrm{E}+04 & 0.30671713 \mathrm{E}+01 & 0.33467176 \mathrm{E}+01 & 0.34548617 \mathrm{E}-02-0.36136810 \mathrm{E}-05\end{array}$

$0.13227441 \mathrm{E}-08-0.66440849 \mathrm{E}-14-0.11213928 \mathrm{E}+040.68718143 \mathrm{E}+01$

CI2+ $112293 \mathrm{C} \quad 2 \mathrm{E}-1 \quad 0 \quad$ OG $300.000 \quad 4000.0001000 .00$

$0.41252842 \mathrm{E}+01 \quad 0.68196526 \mathrm{E}-03-0.40838054 \mathrm{E}-06 \quad 0.11656516 \mathrm{E}-09-0.11435189 \mathrm{E}-13$

$\begin{array}{llll}0.13183581 \mathrm{E}+06 & 0.30671713 \mathrm{E}+01 & 0.33467176 \mathrm{E}+01 & 0.34548617 \mathrm{E}-02-0.36136810 \mathrm{E}-05\end{array}$

$0.13227441 \mathrm{E}-08-0.66440849 \mathrm{E}-14 \quad 0.13199384 \mathrm{E}+06 \quad 0.68718143 \mathrm{E}+01$

END

REACIIONS KELVIN MDIRCULES

! reaction rates from Maxwell ian EEDF

$\mathrm{E}+\mathrm{CL} 2 \Rightarrow \mathrm{E}+\mathrm{C2} \quad 2.5141 \mathrm{E}-02 \quad-1.4443 \mathrm{E}+00 \quad 1.6650 \mathrm{E}+04$ TDEP/E/ !vibrational excitation

EXCI/ $0.07 /$

IUP

$\mathrm{E}+\mathrm{CL} 2 \Rightarrow \mathrm{Cl}-+\mathrm{CL} \quad 5.8901 \mathrm{E}-09 \quad-2.5619 \mathrm{E}-01 \quad 1.5834 \mathrm{E}+04$

TDEP/E/ !dissociative attachent

$\mathrm{E}+\mathrm{CL} 2 \Rightarrow 2 \mathrm{CL}+\mathrm{E} \quad 1.5356 \mathrm{E}-06 \quad-3.4642 \mathrm{E}-01 \quad 7.0850 \mathrm{E}+04$

TDEP/E/ !dissociation

$\mathrm{E}+\mathrm{CL} 2 \Rightarrow \mathrm{E}+\mathrm{CL} 2 \quad 6.3477 \mathrm{E}-06 \quad-5.3987 \mathrm{E}-01 \quad 1.3920 \mathrm{E}+05$

TDEP/E./ !electronic excitation

EXCI / $9.25 /$

DUP

$\mathrm{E}+\mathrm{CL2} \Rightarrow \mathrm{CL} 2++2 \mathrm{E} \quad 1.1227 \mathrm{E}-04 \quad-6.0067 \mathrm{E}-01$ TDEP/E/ !ionization

$\mathrm{E}+\mathrm{CL}-\Rightarrow \mathrm{CL}+2 \mathrm{E}$
$\mathrm{TDEP} / \mathrm{E} / \mathrm{l} /$ detachment

$\begin{array}{ccccc}E+C \underset{\mathrm{TEP} / \mathrm{E} /}{\Rightarrow} & \mathrm{E}+\underset{14 \mathrm{~s} \text { excitation }}{\mathrm{C}^{*}} \quad 1.2363 \mathrm{E}-05 & -6.1356 \mathrm{E}-01 & 1.3297 \mathrm{E}+05\end{array}$

$E+C L \Rightarrow E+C L \quad 1.2363 E-05 \quad-6.1356 \mathrm{E}-01 \quad 1.3297 \mathrm{E}+05$

TDEP/E/ ! Is excitation energy loss

EXCI/ $9.55 /$

DUP 


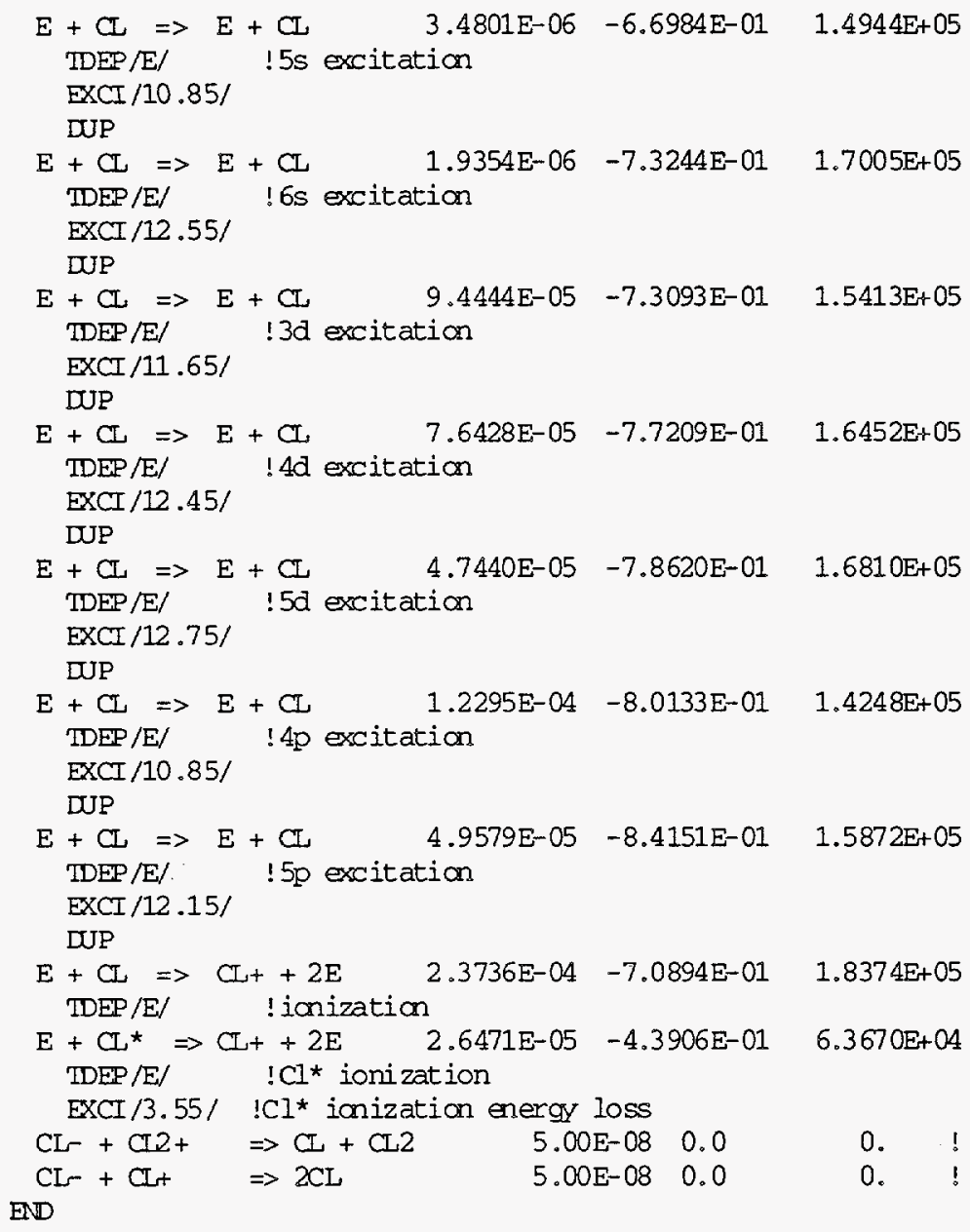




\section{SURFACE CHEMKIN Input for the $\mathrm{Cl}_{2}$ Plasma Example}

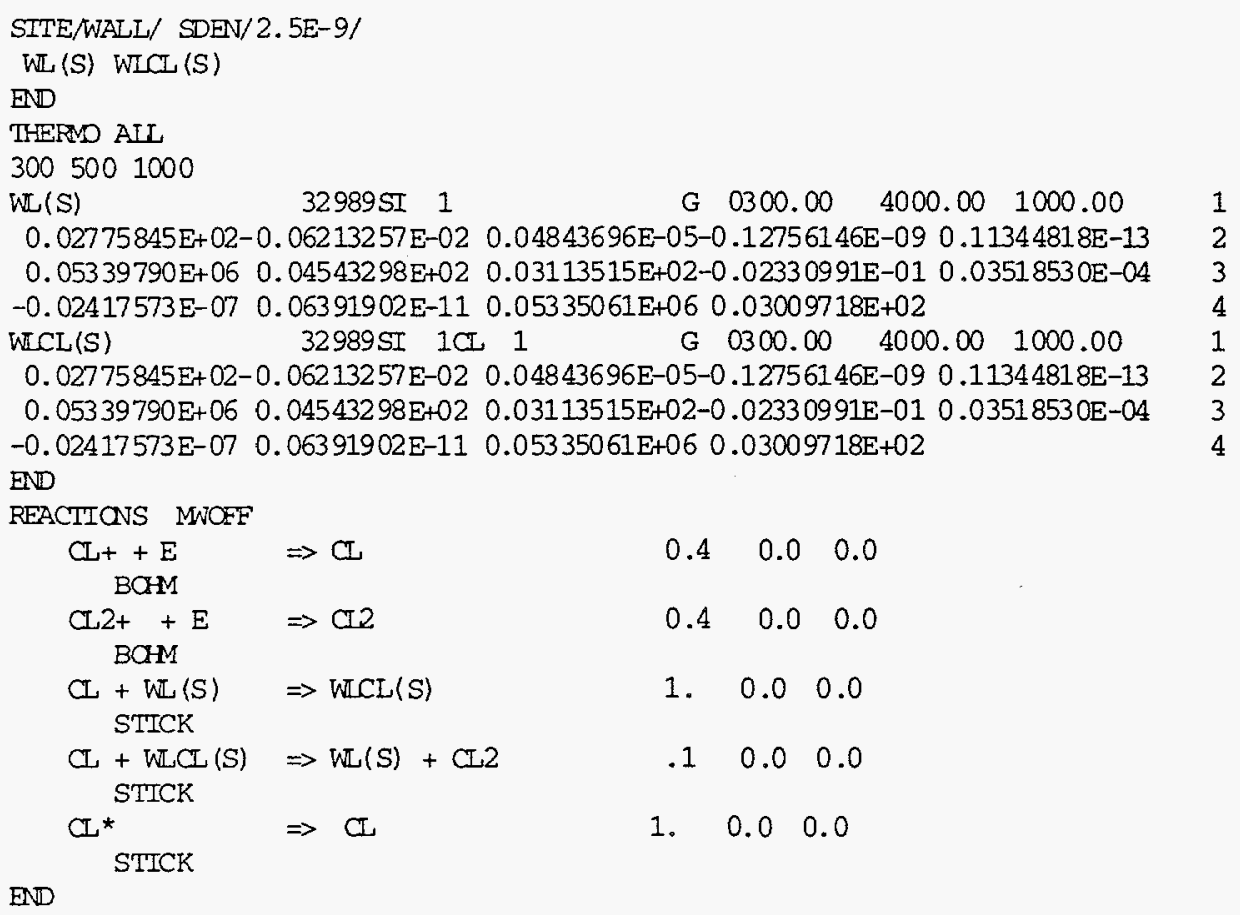




\section{AURORA Output for the $\mathrm{Cl}_{2}$ Plasma Example}

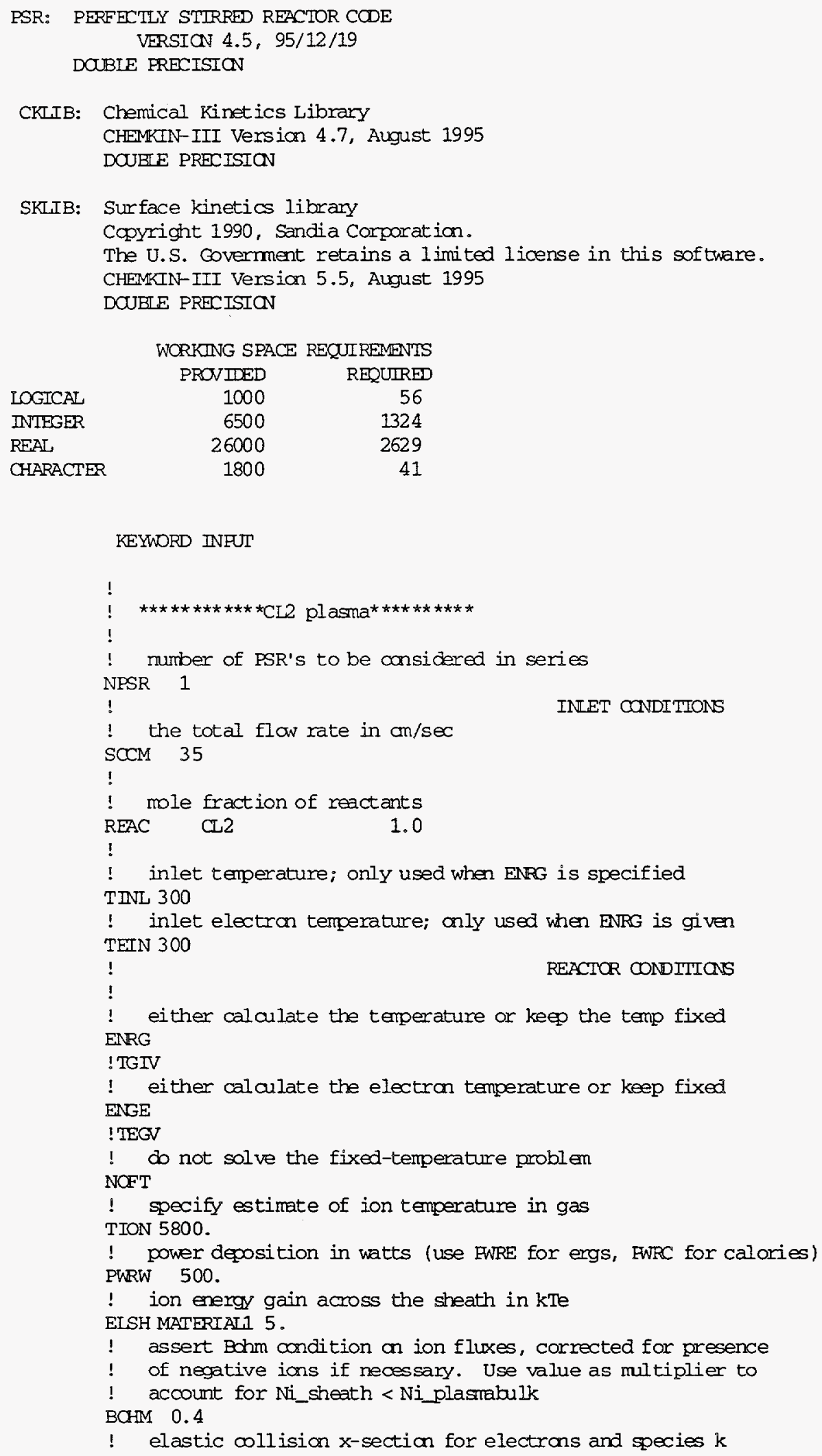




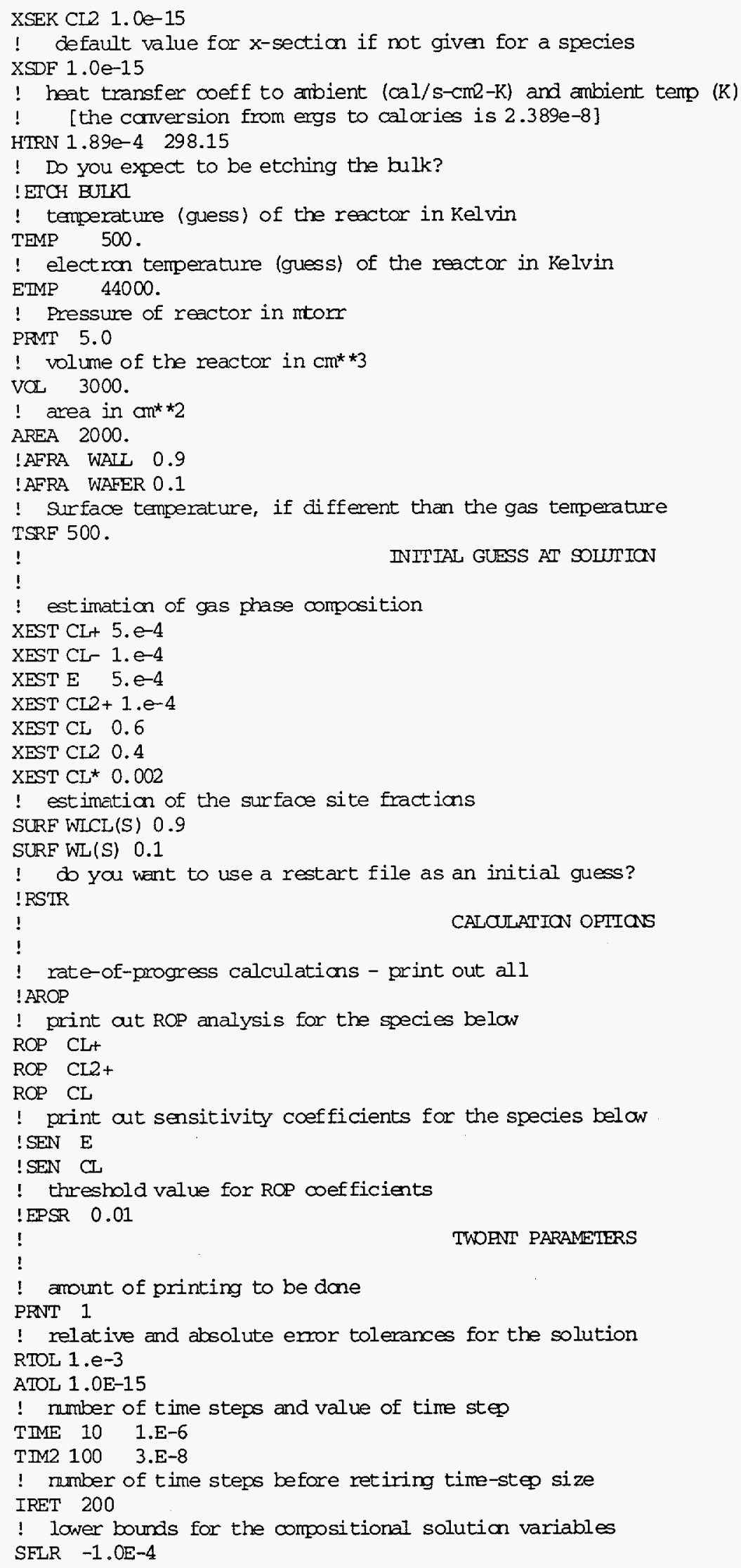


! parameters for the calculation of mumerical differences

ABSL 1.0E-15

RELT . $1 . e-3$

!

! DO YOU WANT TO DO ANOTHER RROBLEM AFIER THIS ONE ?

! $\mathrm{NTN}$

!

END

END OF INPUT PARAMEIERS FOR OURENT PRCELEM

CAUTION. . XEST MOEE FRACTIONS SUM TO 1.003200000000000 FCR BHASE

$1=$ GAS
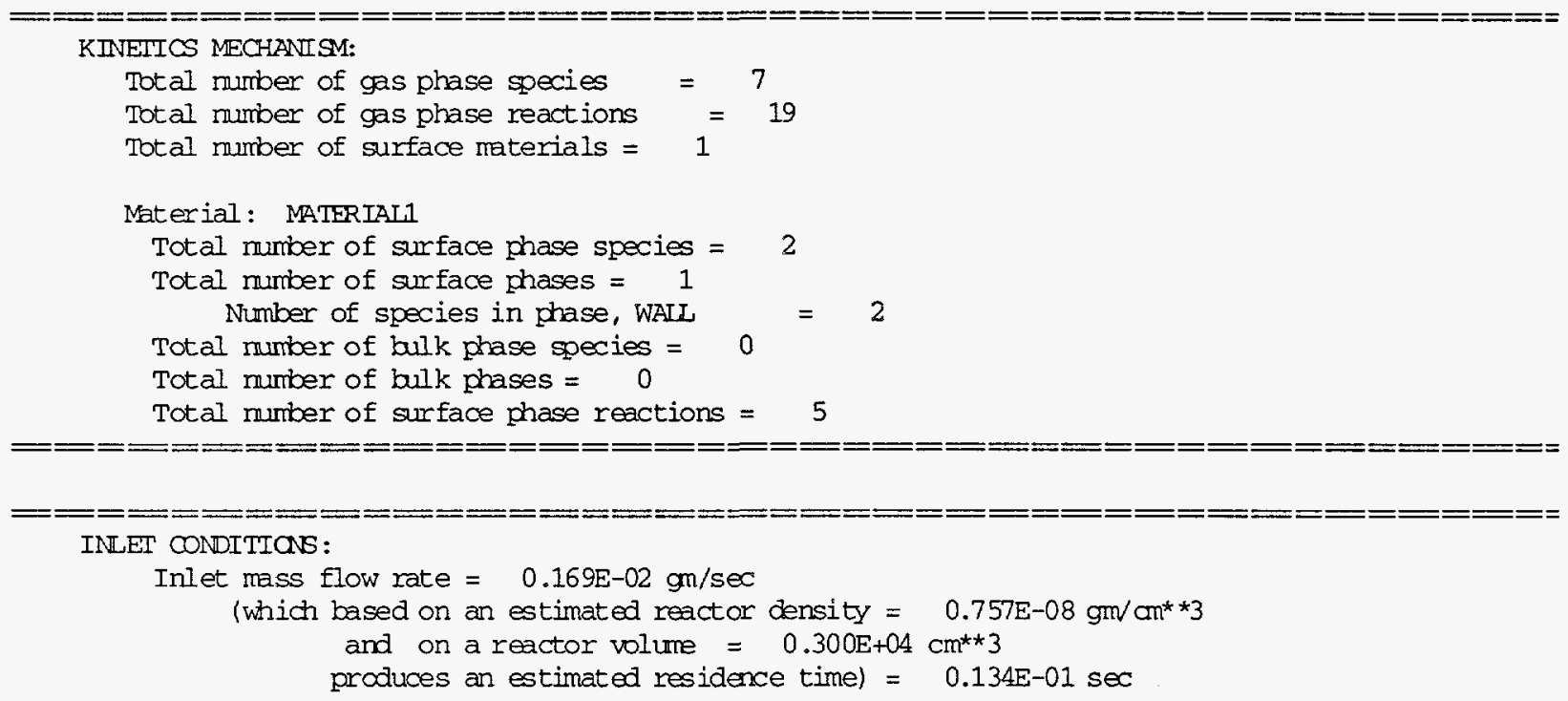

Inlet temperature $=300.00$ Kelvin

Inlet pressure (assumed equal to reactor pressure) $=0.658 \mathrm{E}-05 \mathrm{~atm}$

Inlet density $=0.18950 \mathrm{E}-07 \mathrm{gm} / \mathrm{cm}^{\star *}$

Inlet mean molecular weight $=70.906 \mathrm{gm} / \mathrm{mole}$

Inlet molar flow rate $\quad=0.23843 \mathrm{E}-04 \mathrm{moles} / \mathrm{sec}$

Inlet volumetric flow rate $=89217 . \quad \mathrm{cm}^{*} * 3 / \mathrm{sec}$

(based on reactor pressure and inlet temperature)

$$
\begin{aligned}
& =35.000 \text { SCCM } \\
& =0.35000 \mathrm{E}-01 \text { SLPM }
\end{aligned}
$$

Inlet electron temperature $=300.00 \quad$ Kelvin

$=0.25875 \mathrm{E}-01 \mathrm{eV}$

\begin{tabular}{|c|c|c|c|c|c|c|}
\hline Species & mole_frac & moles/sec & $\mathrm{gm} / \mathrm{sec}$ & $\mathrm{Cm}^{\star \star} \star 3 / \mathrm{sec}$ & $S C C M$ & SLRM \\
\hline $\mathrm{E}$ & $0.00000 \mathrm{E}+00$ & $0.00000 \mathrm{E}+00$ & $0.00000 \mathrm{E}+00$ & $0.00000 \mathrm{E}+00$ & $0.00000 \mathrm{E}+00$ & $0.00000 E+00$ \\
\hline$C++$ & $0.00000 \mathrm{E}+00$ & $0.00000 \mathrm{E}+00$ & $0.00000 \mathrm{E}+00$ & $0.00000 \mathrm{E}+00$ & $0.00000 \mathrm{E}+00$ & $0.00000 \mathrm{E}+00$ \\
\hline $\mathrm{CL} 2+$ & $0.00000 \mathrm{E}+00$ & $0.00000 \mathrm{E}+00$ & $0.00000 \mathrm{E}+00$ & $0.00000 E+00$ & $0.00000 \mathrm{E}+00$ & $0.00000 E+00$ \\
\hline$C-$ & $0.00000 \mathrm{E}+00$ & $0.00000 \mathrm{E}+00$ & $0.00000 \mathrm{E}+00$ & $0.00000 E+00$ & $0.00000 \mathrm{E}+00$ & $0.00000 E+00$ \\
\hline$C^{*}$ & $0.00000 \mathrm{E}+00$ & $0.00000 \mathrm{E}+00$ & $0.00000 \mathrm{E}+00$ & $0.00000 \mathrm{E}+00$ & $0.00000 \mathrm{E}+00$ & $0.00000 \mathrm{E}+00$ \\
\hline$C L$ & $0.00000 \mathrm{E}+00$ & $0.00000 \mathrm{E}+00$ & $0.00000 \mathrm{E}+00$ & $0.00000 \mathrm{E}+00$ & $0.00000 \mathrm{E}+00$ & $0.00000 \mathrm{E}+00$ \\
\hline $\mathrm{CL}_{2}$ & 1.0000 & $0.23843 \mathrm{E}-04$ & $0.16906 \mathrm{E}-02$ & 89217. & 35.000 & $0.35000 \mathrm{E}-01$ \\
\hline
\end{tabular}

INLET CONDITIONS FOR GAS PHASE MOLECULAR SPECTES:

PSRTWO: FINISHED FIXED TEMPERATURE, ADDING ENERGY EQTATION 


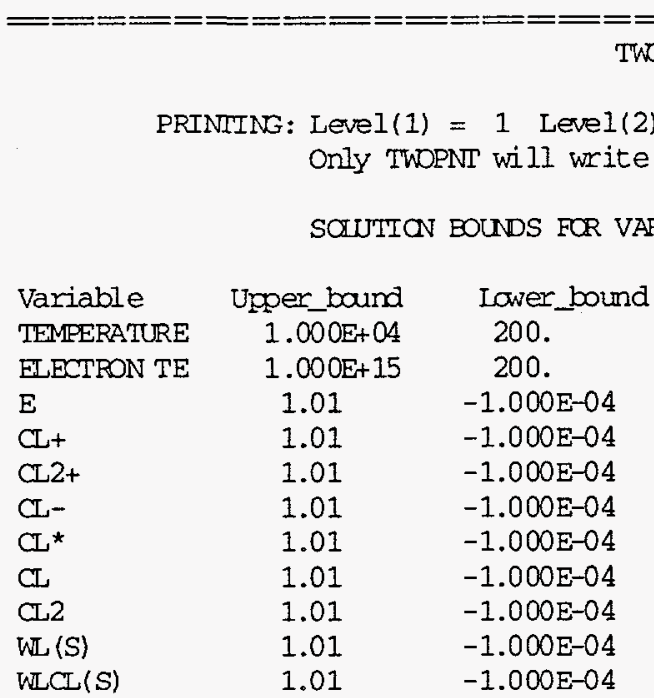

Maxinum number of time steps to try if newton's method fails $=100$

Absolute bound for the steady-state problem $=1.000 \mathrm{E}-15$

Relative bound for the steady-state problem $=1.000 \mathrm{E}-03$

Alosolute bound for the time-dependent prob $=1.000 \mathrm{E}-09$

Relative bound for the time-dependent prob $=1.000 \mathrm{E}-04$

Number of steps to be taken before increasing the time step $=200$

Factor by which to decrease time-step when necessary $=2.20$

Factor by which to increase time-step when necessary $=2.00$

Mininum time step allowed $=1.000 \mathrm{E}-10$

Initial time step $=3.000 \mathrm{E}-08$

Absolute delta for mumerical differencing $=1.000 \mathrm{E}-15$

Relative delta for mumerical differencing $=1.000 \mathrm{E}-03$

TWOANT: DOUBIE PRECISION (TWD POINT BOTNDARY VAIUE PRCBLEM) SOLVER, VERSION 3.18 OF DECEMBER 1995 BY DR. JOSEPH F. GRCAR.

TWOENT: INITIAL GUESS:

PSPRNI: Printing of arrent solution from TWOANT:

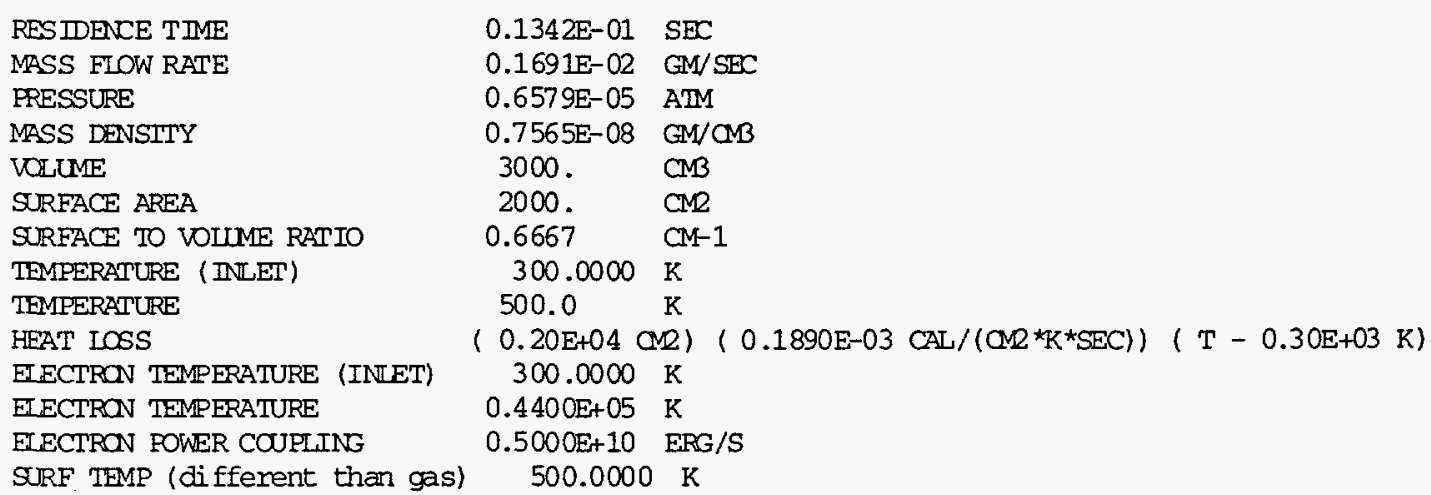


INLET GAS PHASE MOLE FRACTIONS

$\begin{array}{llllll}\mathrm{E} & =0.0000 \mathrm{E}+00 & \mathrm{CL}+ & =0.0000 \mathrm{E}+00 & \mathrm{CL} 2+ & =0.0000 \mathrm{E}+00 \\ \mathrm{CL}- & =0.0000 \mathrm{E}+00 & \mathrm{CL}^{*} & =0.0000 \mathrm{E}+00 & \mathrm{CL} & =0.0000 \mathrm{E}+00 \\ \mathrm{CL} 2 & =1.000 & & & & \end{array}$

EXIT GAS PHASE MOTE FRACTIONS

$\mathrm{E} \quad=0.4984 \mathrm{E}-03$

CL- $\quad=0.9968 \mathrm{E}-04$

व.2 $=0.3987$
CIt
$=0.4984 \mathrm{E}-03$
$\mathrm{CL} 2+$
$=0.9968 \mathrm{E}-04$
$\mathrm{CL}^{\star}$
$=0.1994 \mathrm{E}-02$
$\mathrm{CL}$
$=0.5981$

SURFACE SITE FRACTIONS IN SURFACE BHASE, WALL Site density $=0.2500 \mathrm{E}-08 \mathrm{~mole} / \mathrm{cm}^{\star} \star 2$ Standard state Site density $=0.2500 \mathrm{E}-08 \mathrm{~mole} / \mathrm{am}^{\star *} 2$

Rate of change of site density $=0.0000 \mathrm{E}+00 \mathrm{~mole} /\left(\mathrm{cm}^{\star \star} 2^{\star} \mathrm{Sec}\right)$
WL (S)
$=0.1000$
WLCL (S)
$=0.9000$

CHAREE IMBALANCE FOR THIS SOLUITCN $=0.00000 \mathrm{E}+00 \%$ OF POSITIVE CHARGE DENSITY

PRINTOUT OF RAN RESTDUALS:

Temperature : $-0.142 E+08$

Electron temperature : $0.804 \mathrm{E}-03$

Gas phase mass fraction residuals:

$\mathrm{E} \quad$ : $0.250 \mathrm{E}+10$

CLt : 18.9

$\mathrm{CL} 2+\quad: \quad 72.4$

CLr : 3.18

$C L^{*} \quad:-0.559 \mathrm{E}-01$

CL : -573 .

CI2 : 479 .

Surface phase site fraction residuals:

$\mathrm{WL}(\mathrm{S}) \quad: \quad-4.99$

WLCL(S) : 4.99

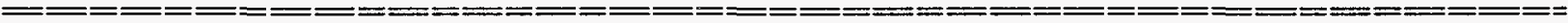

TWOPNT: SOLVE THE PRCBLEM.

$\begin{array}{rrrl} & \text { LOGIO } & \text { LOG10 } & \\ \text { TASK } & \text { NDRM F } & \text { COND J } & \text { REMARK } \\ & & & \\ \text { START } & 9.40 & & \\ \text { SEARCH } & 5.01 & 5.83 & 13 \text { SEARCH STEPS }\end{array}$

TWOENT: FINAL SOLUTION: 


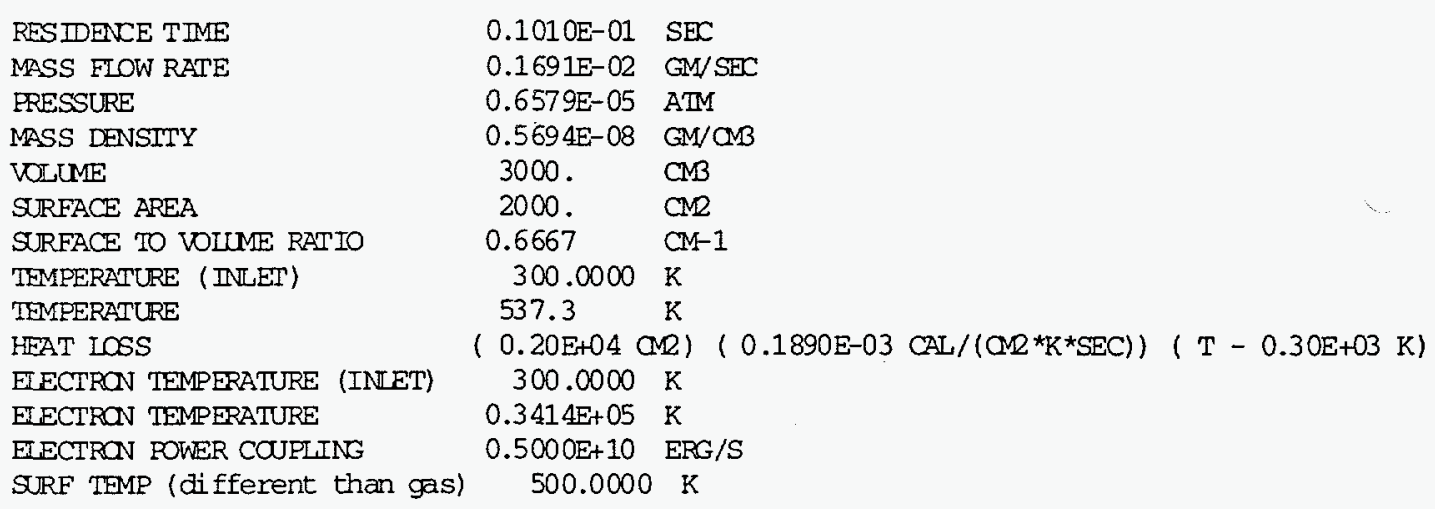

EXIT GAS PHASE MOLE ERACTIONS

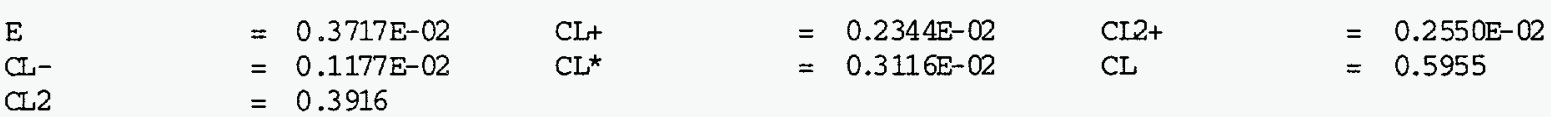

SURFACE SITE FRACTIONS IN SURFACE FHASE, WALL

Site density $=0.2500 \mathrm{E}-08 \mathrm{~mole} / \mathrm{cm}^{\star *} 2$

Standard State Site density $=0.2500 \mathrm{E}-08 \mathrm{~mole} / \mathrm{cm}^{\star *} 2$

Rate of change of site density $=0.0000 \mathrm{E}+00 \mathrm{~mole} /\left(\mathrm{cm}^{\star *} 2^{\star} \mathrm{sec}\right)$

WU (S)

$=0.9091 \mathrm{E}-01 \quad$ WLCL $(\mathrm{S}) \quad=0.9091$

CHARCE IMBALANCE FOR THIS SOLUTICN $=0.13962 E-03:$ OF POSTTIVE CHARCE DENSITY

PRTNTOUT OF RAN RESTDUALS:

Temperature : $-0.164 \mathrm{E}+04$

Electron temperature : $-0.196 \mathrm{E}-06$

Gas phase mass fraction residuals:

$\mathrm{E} \quad: \quad 0.101 \mathrm{E}+06$

CLt $\quad:-0.105 \mathrm{E}-01$

$\mathrm{CL2}+\quad:-0.511 \mathrm{E}-02$

CIr $\quad:-0.427 \mathrm{E}-03$

$\mathrm{CL}^{\star} \quad:-0.188 \mathrm{E}-02$

CL $\quad:-0.154 \mathrm{E}-01$

CL2 : $0.333 \mathrm{E}-01$

Surface phase site fraction resiauals:

WL(S) : $-0.133 \mathrm{E}-03$

WLCL(S) $\quad: \quad 0.163 \mathrm{E}-03$

TWOANT: $\quad 0.21$ SECONDS TOTAL COMPUTER TIME (SEE BREAKDONN BETOW). 
SUBTASK

$\begin{array}{rrrr} & & & \\ 47.6 & 33.3 & 0.0 & 19.0 \\ 0.002 & 0.023 & 0.000 & \\ 52 & 3 & 50 & \end{array}$

52
TASK

EVOLVE SEARCH

$0.0 \quad 90.5$

MEAN SECC

QUANTITY

TWOHNT: SUCCESS. PROBLEM SOLVED.

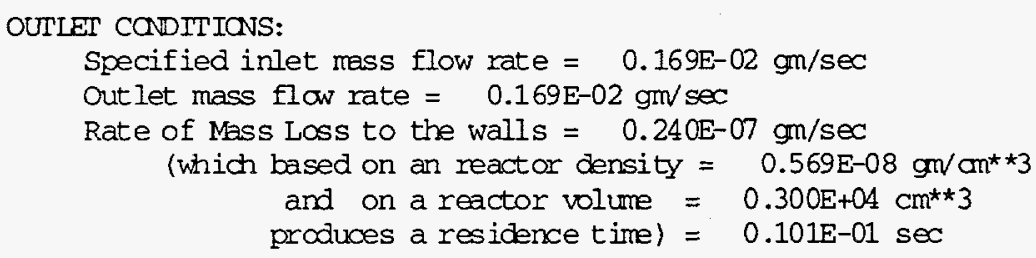

Out let and reactor temperature $=537.31$ Kelvin

Out let and reactor pressure $=0.658 \mathrm{E}-05$ atm out let and reactor density $=0.56935 \mathrm{E}-08 \mathrm{gm} / \mathrm{cm}^{* * 3}$

Out let and reactor mean molecular weight $=49.295$

out let molar flow rate $\quad=0.34296 \mathrm{E}-04 \mathrm{moles} / \mathrm{sec}$

Out let volumetric flow rate $=0.29693 \mathrm{E}+06 \mathrm{~cm} \star * 3 / \mathrm{sec}$

$\mathrm{gm} / \mathrm{mole}$

(based on reactor pressure and temperature)

$$
\begin{array}{ll}
=65.039 & \text { SCCM } \\
=0.65039 \mathrm{E}-01 & \text { SLPM }
\end{array}
$$

Out let and reactor electron temperature $=34139 .^{\circ}$ Kelvin

$$
=2.9445 \mathrm{eV}
$$

\begin{tabular}{|c|c|c|c|c|c|c|}
\hline Species & mole_frac & $\# / \mathrm{cm}^{3}$ & moles/sec & $\mathrm{gm} / \mathrm{sec}$ & $\mathrm{cm}^{\star *} 3 / \mathrm{sec}$ & SOCM \\
\hline $\mathrm{E}$ & $0.37168 \mathrm{E}-02$ & $0.27305 E+12$ & $0.12747 \mathrm{E}-06$ & $0.69928 \mathrm{E}-10$ & 1103.6 & 0.24174 \\
\hline $\mathrm{CL}_{+}$ & $0.23440 \mathrm{E}-02$ & $0.17220 \mathrm{E}+12$ & $0.80389 \mathrm{E}-07$ & $0.28500 \mathrm{E}-05$ & 696.01 & 0.15245 \\
\hline $\mathrm{Cl} 2+$ & $0.25497 \mathrm{E}-02$ & $0.18731 E+12$ & $0.87444 \mathrm{E}-07$ & $0.62003 E-05$ & 757.09 & 0.16583 \\
\hline $\mathcal{C L}_{-}$ & $0.11769 \mathrm{E}-02$ & $0.86460 \mathrm{E}+11$ & $0.40362 \mathrm{E}-07$ & $0.14310 \mathrm{E}-05$ & 349.46 & $0.76544 \mathrm{E}-01$ \\
\hline$C^{*}$ & $0.31161 \mathrm{E}-02$ & $0.22892 \mathrm{E}+12$ & $0.10687 \mathrm{E}-06$ & $0.37889 E-05$ & 925.28 & 0.20267 \\
\hline$C$ & 0.59551 & $0.43749 \mathrm{E}+14$ & $0.20423 \mathrm{E}-04$ & $0.72407 \mathrm{E}-03$ & $0.17683 \mathrm{E}+06$ & 38.731 \\
\hline $\mathrm{CL} 2$ & 0.39159 & $0.28768 \mathrm{E}+14$ & $0.13430 \mathrm{E}-04$ & $0.95225 E-03$ & $0.11627 \mathrm{E}+06$ & 25.468 \\
\hline
\end{tabular}

OUTLET CONDITIONS FOR GAS PHASE MOLECULAR SPECIES:

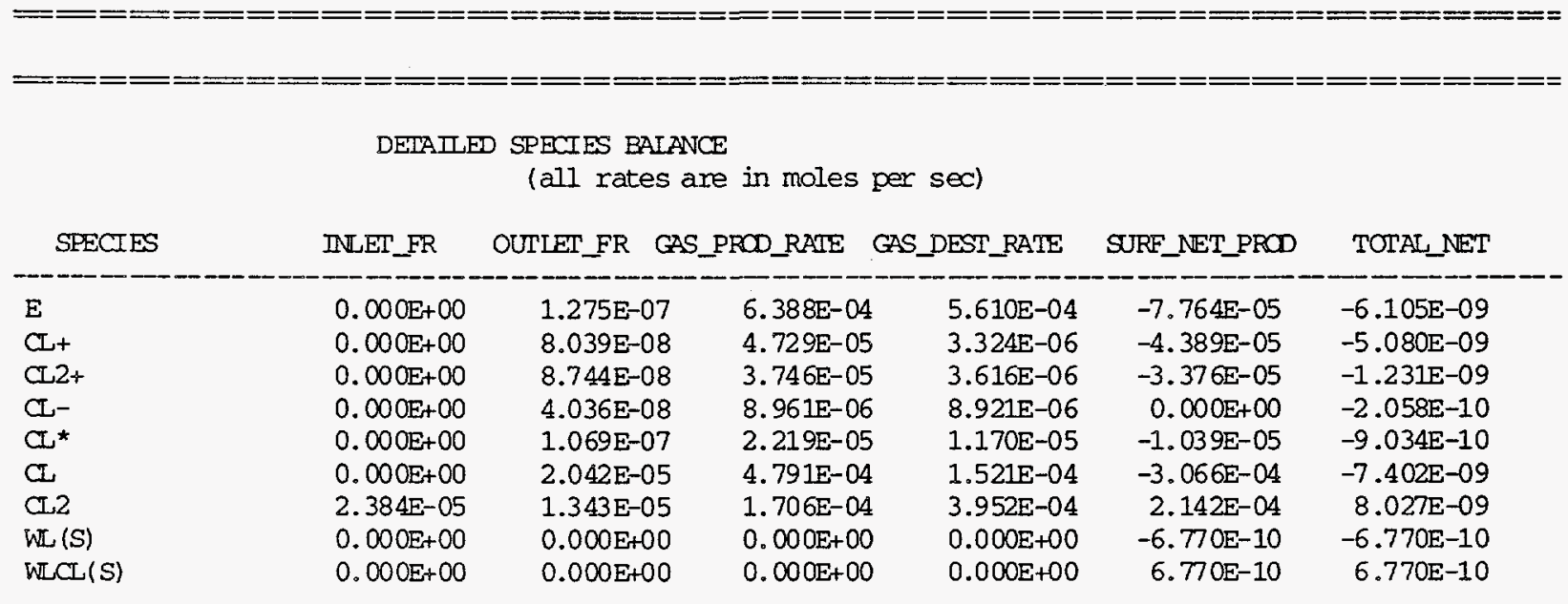


DETAIIED ELLEMENT BAIANCES

(all rates are in moles per sec)

\begin{tabular}{lcrr} 
ELEMENI & TLET_FR & \multicolumn{1}{c}{ OUILET_ER } & TOTAI_NET \\
\hline E & $0.000 \mathrm{E}+00$ & $-2.343 \mathrm{E}-13$ & $2.343 \mathrm{E}-13$ \\
CL & $4.769 \mathrm{E}-05$ & $4.769 \mathrm{E}-05$ & $6.770 \mathrm{E}-10$ \\
SI & $0.000 \mathrm{E}+00$ & $0.000 \mathrm{E}+00$ & $0.000 \mathrm{E}+00$
\end{tabular}

NCRMALIZED AND ABSOLUTE RATE-OF-PROEUCTION COEFFICIENTS

(surface reactions are normalized by the surface area to volume ratio)

Threshold nomalized value for printing of ROP coefficients $=0.100 \mathrm{E}-01$

Gas Phase Species 2. CLt

Gas Phase Reaction 16. E+CL $\Rightarrow C L++2 E$

Gas Phase Reaction 17. E+Cl* $\Rightarrow C L++2 E$

Gas Phase Reaction 19. CL-+Clt $\Rightarrow 2 C \mathrm{CL}$

$\begin{array}{cc}\text { NORMLTZED } & \text { (NOLES/CC-SEC) } \\ 0.753 & (1.1862 \mathrm{E}-08) \\ 0.247 & (3.9006 \mathrm{E}-09) \\ -0.070 & (-1.1081 \mathrm{E}-09) \\ -0.930 & (-1.4629 \mathrm{E}-08)\end{array}$

Total Rate-of-production (moles/ cc-sec) $=1.5762 \mathrm{E}-08$ Total Rate-of-consumption (moles $/ \alpha-\mathrm{sec}$ ) $=1.5737 \mathrm{E}-08$ Net Rate-of-production (moles/oc-sec) $=2.5103 \mathrm{E}-11$

Gas Thase Species 3. CI2+

Gas Phase Reaction 5. E+CL $2 \Rightarrow \mathrm{CL} 2++2 \mathrm{E}$

Gas Phase Reaction 18. $\mathrm{Cl}++\mathrm{Cl} 2+=\mathrm{CCL}+\mathrm{CL} 2$

Surface Phase Reaction 2. $C L 2++E=>C L 2$

Total Rate-of-production (moles/oc-sec) $=1.2486 \mathrm{E}-08$ Total Rate-of-consumption (moles/ oc-sec) $=1.2457 \mathrm{E}-08$ Net Rate-of-production (moles/oc-sec) $=2.8738 \mathrm{E}-11$

Gas thase Species 6. CL

Gas Phase Reaction

Gas Phase Reaction

Gas Phase Reaction

Gas Phase Reaction

Gas Phase Reaction

Surface Phase Reaction

2. $\mathrm{E}+\mathrm{CL} 2 \Rightarrow \mathrm{CL}-+\mathrm{CL}$

3. $\mathrm{E}+\mathrm{CL}, 2 \Rightarrow 2 \mathrm{CL}+\mathrm{E}$

7. $E+C L \Rightarrow E+C L^{\star}$

16. $E+C L=C L L+2 E$

19. $\mathrm{CL}+\mathrm{CL}+\Rightarrow 2 \mathrm{CL}$

1. $\mathrm{CL}+\mathrm{E} \Rightarrow \mathrm{Cl}$

Surface Phase Reaction

3. $C L+W L(S)=>X N L C L(S)$

Surface Phase Reaction

4. $C L+W L C L(S) \Rightarrow W L(S)+C L 2$

Surface Phase Reaction 5. $C^{*}=>C$ C

Total Rate-of-production (moles/cc-sec) $=1.4636 \mathrm{E}-07$

Total Rate-of-consumption (moles/cc-sec) $=1.3955 \mathrm{E}-07$

Net Rate-of-production (moles $/ \propto-\sec$ ) $=6.8054 \mathrm{E}-09$

RATE-OF-ERODUCTION CALCULATION COMPLETE $\begin{array}{cc}\text { NORMALTEDD } & \text { (MDIES/CC-SEC) } \\ 1.000 & (1.2486 \mathrm{E}-08) \\ -0.097 & (-1.2054 \mathrm{E}-09) \\ -0.903 & (-1.1252 \mathrm{E}-08)\end{array}$

NORMAITZED (MOLES/CC-SEC)

$0.020 \quad(2.9870 \mathrm{E}-09)$

0.828 (1.2120E-07)

$-0.053 \quad(-7.3980 \mathrm{E}-09)$

$-0.085 \quad(-1.1862 \mathrm{E}-08)$

$0.015 \quad(2.2162 \mathrm{E}-09)$

$0.100 \quad(1.4629 \mathrm{E}-08)$

$-0.431 \quad(-6.0147 \mathrm{E}-08)$

$0.024 \quad(3.4621 E-09)$
$-0.431 \quad(-6.0147 \mathrm{E}-08)$ 


\section{REFERENCES}

1. Moffat, H. K., Glarborg, P., Kee, R. J., Grcar, J. F. and Miller, J. A., "SURFACE PSR: A Fortran Program for Modeling Well-Stirred Reactors with Gas and Surface Reactions," Sandia National Laboratories Report SAND91-8001, 1991.

2. Kee, R. J., Rupley, F. M., Meeks, E. and Miller, J. A., "Chemkin-III: A Fortran Chemical Kinetics Package for the Analysis of Gas-Phase Chemical and Plasma Kinetics," Sandia National Laboratories Report SAND96-8216, 1996.

3. Coltrin, M. E., Kee, R. J., Rupley, F. M. and Meeks, E., "SURFACE CHEMKIN III: A Fortran Package for Analyzing Heterogeneous Chemical Kinetics at a Solid-Surface - Gas-Phase Interface," Sandia National Laboratories Report SAND96-8217, 1996.

4. Smith, J. M., Chemical Engineering Kinetics (McGraw-Hill Book Company, New York, 1981).

5. Longwell, J. P. and Weiss, M. A., Industrial Engineering and Chemistry 47, 1634 (1955).

6. Bowman, B. R., Pratt, D. T. and Crowe, C. T., "Effect of Turbulent Mixing and Chemical Kinetics on Nitrogen Oxide Production in Jet Stirred Reactors," The Combustion Institute Fourteenth Symposium (International) on Combustion, Pittsburgh, PA, 1973.

7. Pratt, D. T. and Wormeck, J. J., "CREK, A Computer Program for Calculation of Combustion Reaction Equilibrium and Kinetics in Laminar and Turbulent Flow," Washington State University Report WSU-ME-TEL-76-1, 1976.

8. Chen, A. T., Malte, P. C. and Thornton, M. M., "Sulfur-Nitrogen Interaction in Stirred Flames," The Combustion Institute Twentieth Symposium (International) on Combustion, Pittsburgh, PA, 1985.

9. Nenniger, J. E., Kridiotis, A., Chomiak, J., Longwell, J. P. and Sarofim, A. F., The Combustion Institute Twentieth Symposium (International) on Combustion, Pittsburgh, PA, 1985.

10. Glarborg, P., Kee, R. J., Grcar, J. and Miller, J. A., "PSR: A Fortran Program for Modeling WellStirred Reactors," Sandia National Laboratories Report SAND86-8209, 1986.

11. Glarborg, P., Kee, R. J. and Miller, J. A., "Kinetic Modeling and Sensitivity Analysis on Nitrogen Oxide Formation in Well Stirred Reactors," Combustion and Flame 65, 177 (1986). 
12. Coltrin, M. E., Kee, R. J. and Rupley, F. M., "SURFACE CHEMKIN (Version 4.0): A Fortran Package for Analyzing Heterogeneous Chemical Kinetics at a Solid-Surface - Gas-Phase Interface," Sandia National Laboratories Report SAND90-8003C, 1991.

13. Coltrin, M. E., Kee, R. J. and Rupley, F. M., "Surface Chemkin: A General Formalism and Software for Analyzing Heterogeneous Chemical Kinetics at a Gas-Surface Interface," International Journal of Chemical Kinetics 23, 1111 (1991).

14. Ho, P., Breiland, W., Coltrin, M., Moffat, H. and Buss, R., "CVD Process Chemistry," Semiconductor Equipment Technology Center (SETEC) Final Report Vol. II Report SETEC92-035, 1992.

15. Rogoff, G. L., Kramer, J. M. and Piejak, R. B., "A model for the bulk plasma in an RF chlorine discharge," IEEE Trans. on Plasma Sci. PS-14, 103 (1986).

16. Deshmukh, S. C. and Economou, D. J., "Factors affecting the $\mathrm{Cl}$ atom density in a chlorine discharge," J. Appl. Phys. 72, 4597 (1992).

17. Lee, C., Graves, D. B., Lieberman, M. A. and Hess, D. W., "Global Model of Plasma Chemistry in a High Density Oxygen Discharge," Journal of the Electrochemical Society 141, 1546 (1993).

18. Lieberman, M. A. and Gottscho, R. A., in Physics of Thin Films, edited by M. Francombe and J. Vossen (Academic Press, New York, 1993).

19. Meeks, E., Larson, R. S., Shon, J. W. and Vosen, S. R., "Chemistry Modeling in Downstream Etch Systems From the Plasma Source to the Etch Chamber," The Electrochemical Society, Inc. 95-4, Electrochemical Society Symposium on Process Control, Diagnostics, and Modeling in Semiconductor Manufacturing, Reno, Nevada, 1995.

20. Meeks, E. and Shon, J. W., "Effects of Atomic Chlorine Wall Recombination: Comparison of a Plasma Chemistry Model with Experiment," Journal of Vacuum Science and Technology A 13, 2884 (1995).

21. Meeks, E. and Shon, J. W., "Modeling of Plasma-Etch Processes Using Well Stirred Reactor Approximations and Including Complex Gas-Phase and Surface Reactions," IEEE Transactions on Plasma Science 23, 539 (1995).

22. Grcar, J. F., "The Twopnt Program for Boundary Value Problems," Sandia National Laboratories Report SAND91-8230, 1991. 
23. Kee, R. J., Grcar, J. F., Smooke, M. D. and Miller, J. A., "A Fortran Program for Modeling Steady Laminar One-Dimensional Premixed Flames," Sandia National Laboratories Report SAND85$8240,1985$.

24. Coltrin, M. E., Kee, R. J. and Evans, G. H., "A Mathematical Model of the Fluid Mechanics and GasPhase Chemistry in a Rotating Disk Chemical Vapor Deposition Reactor," Journal of the Electrochemical Society 136, 819 (1989).

25. Coltrin, M. E., Kee, R. J., Evans, G. H., Meeks, E., Rupley, F. M. and Grcar, J. F., "SPIN (Version 3.83): A Fortran Program for Modeling One-Dimensional Rotating-Disk/Stagnation-Flow Chemical Vapor Deposition Reactors," Sandia National Laboratories Report SAND91-8003, 1991.

26. Jones, A. and Prothero, A., Combustion and Flame 12, 457 (1968).

27. Engleman, V. S., Bartok, W., Longwell, J. P. and Edelman, R. B., The Combustion Institute Fourteenth Symposium (International) on Combustion, Pittsburgh, PA, 1973.

28. Braithwaite, N. S. J. and Allen, J. E., "Boundaries and Probes in Electronegative Plasmas," Journal of Physics D 21, 1733 (1988).

29. Kee, R. J., Rupley, F. M. and Miller, J. A., "The Chemkin Thermodynamic Data Base," Sandia National Laboratories Report SAND87-8215B, 1990.

30. Riley, M. E., "Unified Model of the rf Plasma Sheath," Sandia National Laboratories Report SAND95-0775, 1995.

31. Reynolds, W. C., "The Element Potential Method for Chemical Equilibrium Analysis: Implementation in the Interactive Program STANJAN," Department of Mechanical Engineering, Stanford University Report 1986.

32. Deuflhard, P., "A Modified Newton Method for the Solution of Ill-Conditioned Systems of Nonlinear Equations with Application to Multiple Shooting," Numerical Mathematics 22, 289 (1974).

33. Hindmarsh, A. C., "LSODE and LSODI, Two New Initial Value Differential Equation Solvers," ACM SIGNUM Newsletter 15, 4 (1980).

34. Stewart, W. E. and Sørensen, J. P., "Sensitivity and Regression of Multicomponent Reactor Models," Dechema 4th International / 6th European Symposium on Chemical Reactor Engineering, Frankfurt, 1976. 
35. Brown, R. A., Scriven, L. E. and Silliman, W. J., in Nonlinear Problems in Dynamics (P. Holmes, Ed.), SIAM, Philadelphia, 1980, Vol. p. 289.

36. Saito, H. and Scriven, L. E., "Study of Coating Flow by the Finite Element Method," Journal of Computational Physics 42, 53 (1981).

37. Miller, J. A., Branch, M. C., McLean, W. J., Chandler, D. W., Smooke, M. D. and Kee, R. J., "The Conversion of $\mathrm{HCN}$ to $\mathrm{NO}$ and $\mathrm{N}_{2}$ in $\mathrm{H}_{2}-\mathrm{O}_{2}-\mathrm{HCN}$-Ar Flames at Low Pressure," The Combustion Institute Twentieth Symposium (International) on Combustion, Pittsburgh, PA, 1985.

38. Dongarra, J. J., Moler, C. B., Bunch, J. R. and Stewart, G. W., LINPACK User's Guide (Society of Industrial and Applied Mathematics, Philadelphia, 1979).

39. Grcar, J. F., "The Change Tool for Change Programs and Scripts," Sandia National Laboratories Report SAND92-8225, 1992.

40. Motz, H. and Wise, H., Journal of Chemical Physics 31, 1893 (1960). 


\section{APPENDIX A. Example Script for Running CHEMKIN, SURFACE CHEMKIN, and AURORA}

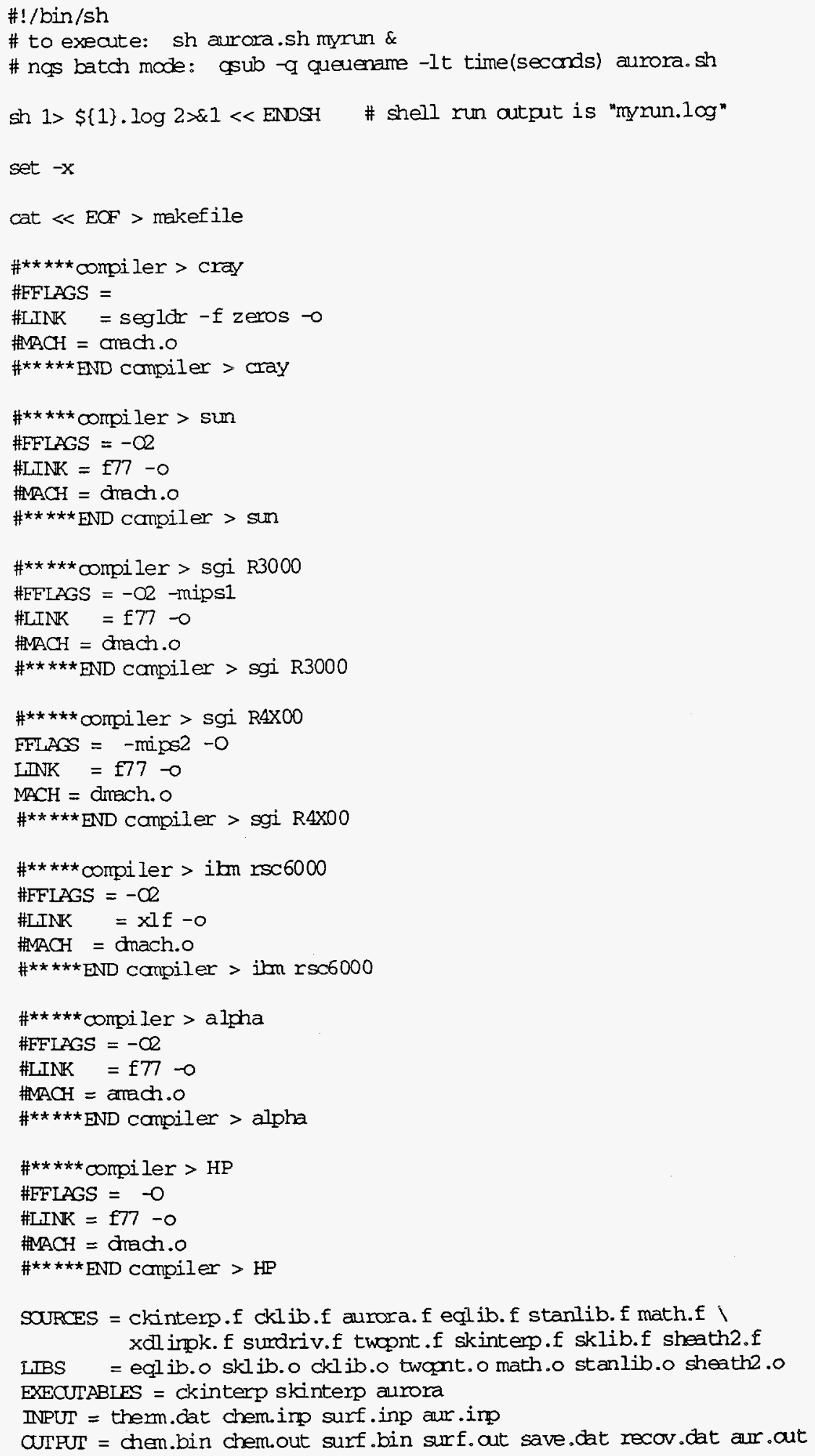


ckinterp : ckinterp.o

\$(LINK) ckinterp ckinterp.o

skinterp : skinterp.o cklib.o

\$ (LINK) skinterp skinterp.o cklib.o

cklib.o : cklib.f ckstrt.h

sklib.o: sklib.f skstrt.h

aurora : surdriv.o aurora. 0 xdlimp. 0 \$ (LIBS)

\$(LIIK) aurora surdriv.o aurora.o xalinpk.o \$(LIBS)

EOF

\#

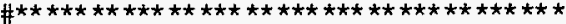

\# Compile and link executables

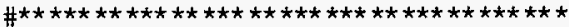

$\#$

touch makefile

make ckinterp

make skinterp

make aurora

make epost

\#

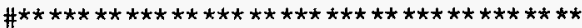

\# Run gas-phase Chemkin interpreter

\# the imput file is 'chem.imp'

\# the output file is 'chem. out'

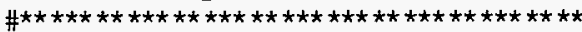

\#

ckinterp

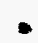

\#

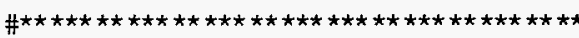

\# Run Surface Chemkin interpreter

\# the input file is 'surf.imp'

\# the output file is 'surf. out'

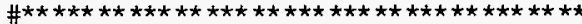

\#

skintemp

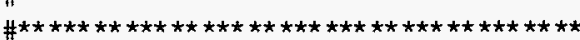

\# Run aurora

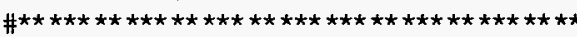

\#

m aur.out save.dat recov. dat;

aurora <aur. inp >aur. out

ENDSH 
INITIAL DISTRIBUTION

UNLIMITED RELEASE

N. Alvi

SEMATECH

2706 Montopolis Drive

Austin, TX 78741

E. Aydil

University of California at Santa Barbara

Department of Chemical and Nuclear Engineering

Santa Barbara, CA 93106

J. N. Bardsley

Mail Stop L296

Lawrence Livermore National Laboratory

P. O. Box 808

Livermore, CAT 94550

D. Beale

Lam Research Corporation

4650 Cushing Parkway

Fremont, CA 94538-6401

L. A. Berry

Oak Ridge National Laboratory

Fusion Energy Division

P. O. Box 2009

Oak Ridge, TN 37831-8071

T. S. Cale

Department of Chemical \& Materials Engineering

Arizona State University

Tempe, AZ 85287

M. A. Cappelli

Mechanical Engineering Department

High Temperature Gas Dynamics Laboratory, Building 520

Stanford University

Stanford, CA 94305-3032

J. L. Cecchi

Department of Chemical \& Nuclear Engineering

University of New Mexico

209 Farris Engineering Center

Albuquerque, NM 87131-1341

C. Cerjan

Mail Stop L296

Lawrence Livermore National Laboratory

P. O. Box 808

Livermore, CA 94550 
E. W. Egan

Motorola

3501 Ed Bluestein Blvd., MD: K-10

Austin, TX 78721

D. Economou

Department of Chemical Engineering

University of Houston

Houston, TX 77204-4792

M. D. Giles

Department of Electrical Engineering

Univeristy of Michigan

1301 Beal Avenue, 1130 EECS Building

Ann Arbor, MI 48109-2122

D. B. Graves

Department of Chemical Engineering

University of California at Berkeley

Berkeley, CA 94720

M. J. Hartig

Motorola

3501 Ed Bluestein Blvd., MD: K-10

- $\quad$ Austin, TX 78721

L. Bromberg

Plasma Fusion Center

Massachusetts Institute of Technology

Cambridge, MA 02139

M. J. Kushner

Department of Electrical and Computer Engineering University of Illinois at Urbana-Champaign

1406 West Green Street

Urbana, IL 61801

A. H. Labun

Digital Equipment Corporation

77 Reed Rd.

M/S HL02-3/J09

Hudson, MA 01749

D. Lessor

K7-15, Building ISB-1

Pacific NW Laboratory

Richmond, WA 99352

D. Mikkelsen

B-249

Princeton Plasma Physics Laboratory

P. O. Box 451

Princeton, NJ 08543-0451 
J. P. McVittie

CIS 133/ Integrated Circuits Laboratory

Stanford University

Stanford, CA 94305-3032

B. Penetrante

Mail Stop L296

Lawrence Livermore National Laboratory

P. O. Box 808

Livermore, CA 94550

Y. Ra

Watkins-Johnson Company

440 Kings Village Road

Scotts Valley, CA 95066-4081

C. Scott

EG3

NASA Johnson Space Center

Houston, TX 77058

S. Shankar

Intel Corporation

Mail Stop RN2-40

2200 Mission College Boulevard

Santa Clara, CA 95052

V. Singh

Lam Research Corporation

4650 Cushing Parkway

Fremont, CA $94538-6470$

Technology Modeling Associates, Inc.

Attn: J. Rey and B. Li

3950 Fabian Way

Palo Also, CA 94303

V. Vahedi

Lam Research Corporation

4650 Cushing Parkway

Fremont, CA 94538-6470

P. A. Vitello

Mail Stop L296

Lawrence Livermore National Laboratory

P. O. Box 808

Livermore, CA 94550

R. Walker

Los Alamos National Laboratory

Group T-12, M. S. B268

Los Alamos, NM 87545 
Y. Ye

Applied Materials

3050 Bowers Avenue

M/S 0225

Santa Clara, CA 95054

0367 R. J. Buss, 1812

0601 P. Esherick, 1126

0601 M. E. Bartram, 1126

0601 W. G. Breiland, 1126

0601 M. E. Coltrin, 1126

0601 J. R. Creighton, 1126

0601 P. Ho, 1126

0601 H. K. Moffat, 1126 (10)

0601 G. H. Hays, 1128

0601 G. A. Hebner, 1128

0601 P. A. Miller, 1128

0601 M. E. Riley, 1128

0826 W. Hermina, 9111

0826 D. K. Gartling, 9111

0827 R. T. McGrath, 9114

0827 T. J. Bartel, 9114

0827 J. E. Brockmann, 9114

0827 R. B. Campbell, 9114

0827 S. J. Choi, 9114

0827 A. S. Geller, 9114

0827 M. L. Hudson, 9114

0827 J. Johannes, 9114

0827 D. J. Rader, 9114

0827 A. J. Russo, 9114

0827 R. Veerasingam, 9114

0827 C. C. Wong, 9114

0841 P. J. Hommert, 9100

Attn: R. D. Skocypec, 9102

J. H. Biffle, 9103

Elaine D. Gorham, 9104

A. C. Ratzel, 9112

S. E. Gianoulakis, 9113

W. H. Rutledge, 9115

C. W. Peterson, 9116

1077 M. G. Blain, 1326

1077 T. L. Meisenheimer, 1326

1077 J. E. Stevens, 1326

1078 C. W. Gwyn, 1302

1078 J. D. McBrayer, 1302

1079 A. D. Romig, 1300

Attn: R. S. Blewer, 1305

G. V. Herrera, 1308

J. Y. Tsao, 1311

L. M. Cecchi, 1326

1111 J. N. Shadid, 1421

1139 M. F. Young, 6513 
1427 P. L. Mattern, 1100

Attn: S. T. Picraux, 1112

J. Nelson, 1113

T. A. Michalske, 1114

G. A. Samara, 1152

E. B. Stechel, 1153

9001 T. Hunter, 8000

Attn: J. B. Wright, 2200

A. West, 8200

R. C. Wayne, 8400

P. N. Smith, 8500

M. T. Dyer, 8700

L. A. Hiles, 8800

9042 C. M. Hartwig, 8345

9042 G. H. Evans, 8345

9042 J. F. Grcar, 8345

9042 S. K. Griffiths, 8345

9042 W. G. Houf, 8345

9042 R. J. Kee, 8303

9042 R. S. Larson, 8345

9042 A. E. Lutz, 8345

9042 E. Meeks, $8345(100)$

9042 R. H. Nilson, 8345

9042 F. M. Rupley, 8345

9042 J. W. Shon, 8345

9042 P. A. Spence, 8345

9042 A. Ting, 8345

9052 M. D. Allendorf, 8361

9053 S. R. Vosen, 8362

9162 D. A. Buchenauer, 8716

9401 L. A. Bertram, 8743

9403 B. E. Mills, 8713

9403 D. K. Ottesen, 8713

9214 C. F. Melius, 8117

9409 R. H. Stulen, 8250

9054 W. J. McLean

Attn: C. W. Robinson, 8301

W. Bauer, 8302

L. A. Rahn, 8351

F. P. Tully, 8353

G. A. Fisk, 8355

D. R. Hardesty, 8361

R. W. Carling, 8362

R. J. Gallagher, 8366

9021 Technical Communications Department, 8535, for OSTI (10)

9021 Technical Communications Department, 8535/Technical Library, MS8099, 13414

8099 Technical Library 13414 (4)

9018 Central Technical Files, 8523-2 (3) 